\title{
Scalar, electromagnetic, and gravitational self-forces in weakly curved spacetimes
}

\author{
Michael J. Pfenning and Eric Poisson \\ Department of Physics, University of Guelph, Guelph, Ontario, Canada N1G 2W1
}

(Revised draft, September 12, 2001)

\begin{abstract}
We calculate the self-force experienced by a point scalar charge $q$, a point electric charge $e$, and a point mass $m$ moving in a weakly curved spacetime characterized by a time-independent Newtonian potential $\Phi$. We assume that the matter distribution responsible for this potential is bounded, so that $\Phi \sim-M / r$ at large distances $r$ from the matter, whose total mass is $M$; otherwise, the Newtonian potential is left unspecified. (We use units in which $G=c=1$.) The self-forces are calculated by first computing the retarded Green's functions for scalar, electromagnetic, and (linearized) gravitational fields in the weakly curved spacetime, and then evaluating an integral over the particle's past world line. The self-force typically contains both a conservative and a nonconservative (radiation-reaction) part. For the scalar charge, the conservative part of the self-force is equal to $2 \xi q^{2} M \hat{\boldsymbol{r}} / r^{3}$, where $\xi$ is a dimensionless constant measuring the coupling of the scalar field to the spacetime curvature, and $\hat{\boldsymbol{r}}$ is a unit vector pointing in the radial direction. For the electric charge, the conservative part of the self-force is $e^{2} M \hat{\boldsymbol{r}} / r^{3}$. For the massive particle, the conservative force vanishes. For the scalar charge, the radiation-reaction force is $\frac{1}{3} q^{2} d \boldsymbol{g} / d t$, where $\boldsymbol{g}=-\boldsymbol{\nabla} \Phi$ is the Newtonian gravitational field. For the electric charge, the radiation-reaction force is $\frac{2}{3} e^{2} d \boldsymbol{g} / d t$. For the massive particle, the radiation-reaction force is $-\frac{11}{3} m^{2} d \boldsymbol{g} / d t$. Our result for the gravitational self-force is disturbing: a radiation-reaction force should not appear in the equations of motion at this level of approximation, and it should certainly not give rise to radiation antidamping. In the last section of the paper we prove that while a massive particle in a vacuum spacetime is subjected only to its self-force, it is also subjected to a matter-mediated force when it moves in a spacetime that contains matter; this force originates from the changes in the matter distribution that are induced by the presence of the particle. We show that the matter-mediated force contains a radiation-damping term that precisely cancels out the antidamping contribution from the gravitational self-force. When both forces are combined, the equations of motion are conservative, and they agree with the appropriate limit of the standard post-Newtonian equations of motion.
\end{abstract}

\section{INTRODUCTION AND SUMMARY}

Motion of massive bodies in strong gravitational fields

The problem of determining the motion of $N$ bodies subjected to their mutual gravitational interactions has been a center piece of general relativity from its inception. Work on this started shortly after the introduction of the theory, and in 1938, a firm formulation of the equations of motion was given by Einstein, Infeld, and Hoffmann [1], who provided post-Newtonian corrections to the Newtonian equations of motion. Higher-order corrections were later added [2 [5], and work continues today, with Damour, Jaranowski \& Schäfer [6],7], de Andrade, Blanchet \& Faye [8,9], and Pati \& Will [10] currently computing corrections of third post-Newtonian order. A technical review of this field of research, as of 1987, can be found in Ref. [11]; the early history was described by Havas [12.

While the equations of motion for slowly moving bodies in a weak gravitational field are now very well understood, the same cannot be said of the fast motion of a massive body in a strong gravitational field. The prototypical problem here is to determine the motion of a structureless particle of mass $m$ in the gravitational field of a much more massive black hole. While it is known that the motion is geodesic in the limit $m \rightarrow 0$, the issue is to determine the corrections to geodesic motion that appear when $m$ in nonvanishing. An important effect that must be introduced is the loss of orbital energy and angular momentum to the gravitational radiation emitted by the moving particle; this is effected by an appropriate radiation-reaction force. Another important effect is a conservative correction to the equations of motion, which disappears when $m \rightarrow 0$. Schematically, therefore, the equations of motion will take the form

$$
m u_{; \beta}^{\alpha} u^{\beta}=f_{\text {self }}^{\alpha},
$$

where $u^{\alpha}$ is the particle's four-velocity in the background of the massive black hole, $u_{; \beta}^{\alpha} u^{\beta}$ its acceleration (the semicolon denotes covariant differentiation in the black-hole metric), and $f_{\text {self }}^{\alpha}$ is the self-force. This contains both a conservative and a dissipative (radiation-reaction) component, and it scales as $m^{2}$ in the small-mass limit. (Throughout the paper we work in relativistic units, with $G$ and $c$ both set equal to unity.) 
A useful way to look at Eq. (1.1) is to imagine that the motion is actually geodesic in a spacetime that is not the background spacetime of the massive black hole, but the perturbed spacetime that contains the particle as well. The geodesic motion in the perturbed spacetime can be expressed as a forced motion in the background spacetime, and this gives rise to Eq. (1.1). The implementation of this idea, however, presents some difficulty: If the particle is pointlike, the perturbation diverges at the position of the particle, and the geodesic equation is not defined on the world line. The perturbation must then be decomposed into a part that is singular but does not influence the motion of the particle, and a smooth remainder that is entirely responsible for the self-force [13]. This decomposition requires great care.

In simple situations, the orbital evolution of a particle emitting gravitational waves can be determined without the involvement of a self-force. If the black hole is nonrotating, the orbital evolution is determined by energy and angular-momentum balance [14,15]: The rate at which the radiation carries energy and angular momentum both to infinity and across the event horizon matches the rate at which orbital energy and angular momentum is lost by the particle. This information alone is sufficient to solve for the motion, at least when the time scale for orbital evolution is long compared with the orbital period. If the black hole is rotating, the statement continues to be true provided that the orbit is either equatorial or circular [16 21]. When dealing with generic orbits around a rotating black hole, however, the loss of orbital energy and angular momentum no longer constitutes sufficient information - the rate of change of the "Carter constant" 22] must also be determined, and this requires the involvement of a self-force [20].

\section{Sources of gravitational waves for LISA}

There is a practical necessity for the computation of the gravitational self-force. Solar-mass compact objects in highly eccentric, nonequatorial orbits around rapidly rotating, massive black holes (in the range between $10^{3}$ and $10^{8}$ solar masses) emit gravitational waves that will be measured by eventual spaceborne interferometric detectors [23], which operate in the low-frequency band (in the range between $10^{-4}$ and $1 \mathrm{~Hz}$ ). One such detector, the Laser Interferometer Space Antenna (LISA) [24], has been selected as one of three "Cornerstone missions" in the "Horizon 2000+" program of the European Space Agency. A possible involvement by the National Aeronautics and Space Administration - now appearing likely after the publication of the Taylor-McKee decadal survey [25], in which LISA is listed as a high-priority, moderate-size mission — would increase the likelihood that this detector will be deployed in the not-too-distant future. A realistic projection places the launch in the neighborhood of 2010.

The detailed modeling of these sources of gravitational waves for LISA, to the extent that templates could be provided for data analysis, will require a detailed computation of the orbital evolution. This, in turn, requires that Eq. (1.1) be evaluated and solved for generic orbits around a Kerr black hole. For this we need a practical way of computing the gravitational self-force.

\section{Gravitational self-force}

This issue was taken up recently by Mino, Sasaki, and Tanaka [26], who calculated the self-force acting on a point particle of mass $m$ moving in an arbitrary background spacetime with metric $g_{\alpha \beta}$; they assume that the metric satisfies the Einstein field equations in vacuum. Their calculation is based on a careful analysis of the perturbation field in the vicinity of the particle, the (gravitational) perturbation being created by the particle itself. It involves a careful decomposition of the perturbation into a part that is singular at the particle's location but does not affect its motion, and a part that is well-behaved and governs the motion. Their expression for the self-force was then reproduced by Quinn and Wald [27], on the basis of a different approach involving a comparison between self-forces acting in two different spacetimes. Both teams found the following equations of motion:

$$
m u_{; \beta}^{\alpha} u^{\beta}=f_{\text {ext }}^{\alpha}-\frac{11}{3} m\left(\delta_{\beta}^{\alpha}+u^{\alpha} u_{\beta}\right) \dot{f}_{\text {ext }}^{\beta}+f_{\text {grav }}^{\alpha} .
$$

Here, $u^{\alpha}$ is the particle's four-velocity in the background spacetime, $f_{\text {ext }}^{\alpha}$ is an external force acting on the particle, and an overdot indicates differentiation with respect to proper time $\tau$. The gravitational self-force is given by

$$
f_{\text {grav }}^{\alpha}=-2 m^{2} \int_{-\infty}^{\tau^{-}}\left(2 G_{\beta \mu^{\prime} \nu^{\prime} ; \gamma}^{\alpha}-G_{\beta \gamma \mu^{\prime} \nu^{\prime}} ; \alpha+u^{\alpha} G_{\beta \gamma \mu^{\prime} \nu^{\prime} ; \delta} u^{\delta}\right) u^{\beta} u^{\gamma} u^{\mu^{\prime}} u^{\nu^{\prime}} d \tau^{\prime}
$$

The four-index object $G_{\gamma^{\prime} \delta^{\prime}}^{\alpha \beta}\left(x, x^{\prime}\right)$ appearing inside the integral is the retarded gravitational Green's function [28], whose precise definition is given by Eq. (3.25) below (our normalization differs from the conventional choice by a 
factor of 4); the unprimed indices refer to the field point $x$ (which is identified in the integral with the current position of the particle), while the primed indices refer to the source point $x^{\prime}$ (identified with the particle's past position). The integral extends over the past world line of the particle, from $\tau^{\prime}=-\infty$ to (almost) the current time, $\tau^{\prime}=\tau^{-} \equiv \tau-\epsilon$, where $\epsilon$ is very small and positive [27]. The integration is cut short to avoid the singular behavior of the Green's function as $x^{\prime}$ approaches $x$; it involves only the smooth part of the Green's function, which is often referred to as its "tail part" 229. In a situation in which the particle is subjected only to its self-force, the equations of motion reduce to $m u_{; \beta}^{\alpha} u^{\beta}=f_{\text {grav }}^{\alpha}$. Because the self-force scales like $m^{2}$, we recover geodesic motion in the limit $m \rightarrow 0$.

The difficulty of evaluating Eq. (1.3) resides with the computation of the gravitational Green's function, which would be very hard to carry out for an arbitrary spacetime. There is hope for progress, however, if the spacetime possesses symmetries, such as staticity and spherical symmetry in the case of a Schwarzschild black hole, or stationarity and axial symmetry in the case of a Kerr black hole. In such cases, a plausible method of computation would be based on a separation-of-variables approach, and the modes of the Green's function could be obtained fairly easily [30]. But this approach is not free of difficulties: While the individual modes of the Green's function stay finite and continuous as $x$ approaches $x^{\prime}$ (though they are discontinuous in their first derivatives), the sum over modes does not converge. Essentially, this is because the modes do not distinguish between the singular and smooth parts of the Green's function; they contribute to both, and the singular behavior of the Green's function gives rise to a divergent sum. Recently, Ori, Burko, and Barack [31 37] have devised a way to regularize the mode sums, so as to extract from them a meaningful expression for the self-force. They have applied their technique to a number of simple situations involving scalar, electromagnetic, and gravitational radiation. Similar regularization methods were proposed by Lousto [38], as well as Nakano, Mino, and Sasaki [39,40]. It appears likely that in the near future, this method will be used successfully to solve more complicated problems, including the prototypical problem mentioned previously.

\section{Electromagnetic self-force}

The derivation of Eqs. (1.2) and (1.3) by Mino et al. [26], and Quinn \& Wald [27], is based on methods previously introduced by DeWitt and Brehme [41]. These authors calculated the self-force acting on an electrically charged particle moving in an arbitrary spacetime with metric $g_{\alpha \beta}$. As corrected by Hobbs 42], the equations of motion of a charged particle are

$$
m u_{; \beta}^{\alpha} u^{\beta}=f_{\mathrm{ext}}^{\alpha}+\frac{2}{3} \frac{e^{2}}{m}\left(\delta_{\beta}^{\alpha}+u^{\alpha} u_{\beta}\right) \dot{f}_{\mathrm{ext}}^{\beta}+\frac{1}{3} e^{2}\left(R_{\beta}^{\alpha} u^{\beta}+u^{\alpha} R_{\beta \gamma} u^{\beta} u^{\gamma}\right)+f_{\mathrm{em}}^{\alpha},
$$

where $e$ is the particle's electric charge, $f_{\mathrm{ext}}^{\alpha}$ an external force acting on the particle, $R_{\alpha \beta}$ the spacetime's Ricci tensor, and

$$
f_{\mathrm{em}}^{\alpha}=-e^{2} \int_{-\infty}^{\tau^{-}}\left(G_{\gamma^{\prime} ; \beta}^{\alpha}-G_{\beta \gamma^{\prime}}^{; \alpha}\right) u^{\beta} u^{\gamma^{\prime}} d \tau^{\prime}
$$

is the electromagnetic self-force. The two-index object $G_{\beta^{\prime}}^{\alpha}\left(x, x^{\prime}\right)$ is the retarded electromagnetic Green's function [41], whose precise definition is given by Eq. (3.16) below. In the absence of an external force, and in a region of spacetime empty of matter, the equations of motion reduce to $m u_{; \beta}^{\alpha} u^{\beta}=f_{\mathrm{em}}^{\alpha}$. In flat spacetime, on the other hand, $f_{\mathrm{em}}^{\alpha}=0$ because the Green's function contains only a singular part; the smooth, or tail, part vanishes. In flat spacetime, therefore, we recover the Abrahams-Lorentz-Dirac equation [43 45],

$$
m u_{; \beta}^{\alpha} u^{\beta}=f_{\mathrm{ext}}^{\alpha}+\frac{2}{3} \frac{e^{2}}{m}\left(\delta_{\beta}^{\alpha}+u^{\alpha} u_{\beta}\right) \dot{f}_{\mathrm{ext}}^{\beta} .
$$

The practical computation of the electromagnetic self-force presents the same technical difficulties as in the gravitational case.

\section{Scalar self-force}

The self-force acting on a particle with scalar charge $q$ was recently calculated by Quinn [46]. In this case, the equations of motion are 


$$
m u_{; \beta}^{\alpha} u^{\beta}=f_{\mathrm{ext}}^{\alpha}+\frac{1}{3} \frac{q^{2}}{m}\left(\delta_{\beta}^{\alpha}+u^{\alpha} u_{\beta}\right) \dot{f}_{\mathrm{ext}}^{\beta}+\frac{1}{6} q^{2}\left(R_{\beta}^{\alpha} u^{\beta}+u^{\alpha} R_{\beta \gamma} u^{\beta} u^{\gamma}\right)+f_{\mathrm{scalar}}^{\alpha},
$$

where the self-force is now given by

$$
f_{\text {scalar }}^{\alpha}=q^{2}\left(g^{\alpha \beta}+u^{\alpha} u^{\beta}\right) \int_{-\infty}^{\tau^{-}} G_{, \beta} d \tau^{\prime},
$$

in which the retarded Green's function $G\left(x, x^{\prime}\right)$ is a scalar; this is defined by Eq. (3.10) below. In the absence of an external force, the equations of motion reduce to $m u_{; \beta}^{\alpha} u^{\beta}=f_{\text {scalar }}^{\alpha}$, and this is the simplest realization of the class of equations that govern the orbital evolution of a particle moving in a strong gravitational field. The computation of the scalar, electromagnetic, or gravitational self-force involves solving for a retarded Green's function, and then performing an integration over the particle's past world line. Because there is only one component to the scalar Green's function (as opposed to 16 for the electromagnetic function, or 100 for the gravitational case), Eq. (1.8) captures the essence of the problem and avoids many technical complications. For this reason, it has been the starting point of many recent investigations $32,37,39,40,47$.

\section{Weak-field limit}

The computation of the various Green's functions is a very difficult undertaking even when the spacetime possesses many symmetries. This is, however, a very tractable task when the spacetime is only weakly curved. In such a situation, the metric itself can be calculated perturbatively as an expansion about flat spacetime, and the same technique can be employed to find the Green's functions and compute the self-forces. Our purpose with this paper is to do just that. We will consider spacetimes for which the metric can be expressed as

$$
d s^{2}=-(1+2 \Phi) d t^{2}+(1-2 \Phi)\left(d x^{2}+d y^{2}+d z^{2}\right),
$$

in which $\Phi(x, y, z) \ll 1$ is a generic Newtonian potential satisfying Poisson's equation, $\nabla^{2} \Phi=4 \pi \rho$, where $\rho$ is the mass density. We will keep $\Phi$ unspecified throughout the paper, but assume that it is small everywhere. We shall also assume that the mass distribution is bounded, so that $\Phi$ behaves as $-M / r$ at large distances $r$ from the center of mass; $M=\int \rho d^{3} x$ is the total mass, and $r^{2}=x^{2}+y^{2}+z^{2}$. Various aspects of those spacetimes are discussed in Sec. II. In Sec. III and IV we calculate the scalar, electromagnetic, and gravitational Green's functions to first order in $\Phi$. In Sec. V we use the Green's functions to calculate the scalar, electromagnetic, and gravitational self-forces.

Our motivation for carrying out such a (long) computation is to provide a useful check on the formalism, especially in its gravitational formulation. Admittedly, the weak-field limit is not by itself very interesting, but understanding this limit may well be a necessary step toward understanding the strong-field behavior of the gravitational self-force. So while we cannot hope to learn anything new from such a weak-field computation, we can use our knowledge of the post-Newtonian equations of motion [3 5 .11] to produce a nontrivial check of the self-force method; the results found here should of course match the results from the literature. As we shall see, we have been successful at producing this check: the method works.

\section{DeWitt and DeWitt}

We are not the first to perform such a check on the formalism. In 1964, DeWitt and DeWitt [48] calculated the electromagnetic self-force, as given by Eq. (1.5), for the weakly curved spacetime of Eq. (1.9). They considered only the special case $\Phi(\boldsymbol{x})=-M / r$, where $r=|\boldsymbol{x}|$, but noted that their results generalize to arbitrary potentials by superposition. (We use a bold-faced symbol to denote a three-dimensional vector living in flat space.) They found that to leading order in a weak-field, slow-motion approximation, the spatial components of the self-force are

$$
\boldsymbol{f}_{\mathrm{em}}(\boldsymbol{x})=e^{2} \frac{M}{r^{3}} \hat{\boldsymbol{r}}+\frac{2}{3} e^{2} \frac{d \boldsymbol{g}}{d t},
$$

where $\hat{\boldsymbol{r}}=\boldsymbol{x} / r$ and $\boldsymbol{g}(\boldsymbol{x})=-\boldsymbol{\nabla} \Phi$ is the Newtonian gravitational field. In this limit, the equations of motion $m u_{; \beta}^{\alpha} u^{\beta}=f_{\mathrm{em}}^{\alpha}$ become

$$
m \frac{d^{2} \boldsymbol{z}}{d t^{2}}=m \boldsymbol{g}(\boldsymbol{z})+\boldsymbol{f}_{\mathrm{em}}(\boldsymbol{z})
$$


where $\boldsymbol{z}(t)$ is the trajectory of the charged particle. The first term on the right-hand side of Eq. (1.10) is a repulsive correction to the local force of gravity; it agrees with the weak-field limit of a result by Smith and Will [49], who calculated the force required to hold a charged particle in place in the (exact) field of a Schwarzschild black hole. The second term is the usual expression for the radiation-reaction force experienced by a charged particle subjected to an external force $m \boldsymbol{g}$ [43.44].

The result of Eq. (1.10) is remarkable because this entirely local expression for the self-force derives from a nonlocal formulation involving the entire past history of the charged particle; in the weak-field limit, any trace of nonlocality is lost. The result is remarkable also because while the expression for the radiation-reaction component of the self-force is the expected $\frac{2}{3} e^{2} d \boldsymbol{g} / d t$, it is very hard to see how such a simple result could ever follow from such a complicated expression as Eq. (1.5). We notice that this result for the radiation-reaction force can be obtained from two very different approaches. In a flat-spacetime point of view, the equations of motion for a charged particle are $m u_{; \beta}^{\alpha} u^{\beta}=f_{\text {ext }}^{\alpha}+\frac{2}{3}\left(e^{2} / m\right)\left(\delta_{\beta}^{\alpha}+u^{\alpha} u_{\beta}\right) \dot{f}_{\text {ext }}^{\beta}$; in the slow-motion limit, the external force is $m \boldsymbol{g}$, and we recover the usual result. In a curved-spacetime point of view, on the other hand, there is no external force and the equations of motion are $m u_{; \beta}^{\alpha} u^{\beta}=f_{\mathrm{em}}^{\alpha}$; in the weak-field, slow-motion limit, we also recover the usual result. This agreement is necessary on physical grounds, but it could hardly have been anticipated on the basis of a quick inspection of Eq. (1.5); the calculations required to produce Eq. (1.10) are very involved.

In their paper [48], DeWitt and DeWitt gave the following physical picture for the self-force of Eq. (1.10). This picture relies heavily on their expression for the Newtonian potential, $\Phi=-M / r$, which corresponds to a point mass $M$ located at the origin of the coordinate system.

Because the fundamental expression for the self-force, Eq. (1.5), involves only the smooth, or tail, part of the electromagnetic Green's function, there is a priori no local contribution to this force coming from the world-line integral. Indeed, DeWitt and DeWitt found that the quantity inside this integral vanishes in the immediate past of the event $(t, \boldsymbol{x})$, which corresponds to the particle's current position. In fact, it vanishes until the time delay, $t-t^{\prime}$, becomes equal to $r+r^{\prime}$, the distance between the central mass $M$ and the particle's current position $\boldsymbol{x}$, added to the distance to the particle's old position $\boldsymbol{x}^{\prime}$. At $t-t^{\prime}=r+r^{\prime}$, the electromagnetic Green's function undergoes an abrupt change of behavior, and the self-force is entirely due to this sudden transition. (We expand our discussion of this point in Appendix B.) The physical effect responsible for the force, in DeWitt and DeWitt's view [48], is a signal that originates from the particle at an earlier time $t^{\prime}$, propagates toward the central mass $M$ at the speed of light, bounces off the central mass, and comes back to the particle at the current time $t$. Although the self-force is nonlocal, Eq. (1.10) involves the conditions at the current time only. This is because the time delay in not noticeable at the level of approximation maintained in the calculation. To leading order in a weak-field, slow-motion approximation, the electromagnetic self-force appears to be entirely local.

The physical picture described in the preceding paragraph appeared to us to be slightly suspicious. The sudden change in the behavior of the electromagnetic Green's function at $t-t^{\prime}=r+r^{\prime}$ is dictated by the presence of the point mass $M$ at $\boldsymbol{x}=0$, which mediates the interaction between the charged particle and its earlier self. The region of spacetime near the central mass is therefore seen to have an important effect on the Green's function, and it is this effect - the sudden change - which apparently gives rise to the self-force. But $\Phi=-M / r$ cannot be considered to be small in this region of spacetime, and the perturbative method of calculation of the Green's function must come into question. While there is little reason to doubt the validity of Eq. (1.10), it appeared to us that its derivation by DeWitt and DeWitt [48] left room for improvement.

\section{This paper}

Part of this paper is concerned with providing a derivation of Eq. (1.10) that is free of this criticism. Throughout the paper we assume that $\Phi(\boldsymbol{x})$ is everywhere much smaller than unity, and reduces to $-M / r$ only far away from the mass distribution. Building upon DeWitt and DeWitt's work [48], we introduce (in Secs. III and IV) techniques that allow us to compute the electromagnetic Green's function for such generic Newtonian potentials. In Sec. V we recalculate the electromagnetic self-force for these potentials, and we reproduce Eq. (1.10). While our result is compatible with the physical picture suggested by DeWitt and DeWitt, our derivation shows very clearly that the result is largely independent of the conditions near the mass distribution. We consider this improvement on the original derivation to be a significant contribution to this field. Our derivation is also more transparent, in the sense that the computational labor involved is much reduced compared with the original calculations of DeWitt and DeWitt.

The techniques introduced in Secs. III and IV allow us to calculate all three types of Green's functions — scalar, electromagnetic, and gravitational. Those are used in Sec. V to calculate the respective self-forces. Part of the paper is therefore devoted to the calculation of the scalar and gravitational self-forces in the weakly curved spacetimes of Eq. (1.9). To the best of our knowledge, such calculations have not yet been presented in the literature. 


\section{Scalar self-force}

The computations are simplest for the case of a scalar charge, and we obtain

$$
\boldsymbol{f}_{\text {scalar }}=2 \xi q^{2} \frac{M}{r^{3}} \hat{\boldsymbol{r}}+\frac{1}{3} q^{2} \frac{d \boldsymbol{g}}{d t},
$$

where $\xi$ is a constant measuring the coupling of the scalar field to the spacetime curvature; a precise definition is given by Eq. (2.7) below. The equations of motion for the scalar charge $q$ are identical to Eq. (1.11), but with the scalar selfforce replacing the electromagnetic self-force. For minimal coupling $(\xi=0)$, the self-force is entirely dissipative, and we recover the expected result, $\frac{1}{3} q^{2} d \boldsymbol{g} / d t$; this is the radiation-reaction force experienced by a scalar charge subjected to an external force $m \boldsymbol{g}$. For minimal coupling, the self-force acting on a stationary charge vanishes; this agrees with Wiseman's result [47] for the force required to hold a scalar charge in place in the exact field of a Schwarzschild black hole. If $\xi>0$, the conservative part of the self-force is repulsive. If $\xi<0$, it is attractive. This result for the scalar self-force is very similar to Eq. (1.10), and we notice that the highly nonlocal expression of Eq. (1.8) has managed to produce the expected local result. Once more the dual point of view holds: We can adopt a flat-spacetime point of view, set $f_{\text {scalar }}^{\alpha}$ to zero in Eq. (1.7), and get the correct result by equating the external force to $\mathrm{mg}$; or we can adopt a curved-spacetime point of view, set $f_{\text {ext }}^{\alpha}$ to zero in Eq. (1.7), and get the correct result by evaluating the world-line integral of Eq. (1.8).

\section{Gravitational self-force and matter-mediated force}

The computations required for the gravitational self-force are the most involved, but here we also face a serious technical problem. We have stressed the importance of working with a Newtonian potential that is everywhere small. In fact, the perturbative calculation of the Green's function requires that the deviations of the metric $g_{\alpha \beta}$ with respect to the Minkowski values $\eta_{\alpha \beta}$ be everywhere small. To accommodate this requirement, it is necessary that the spacetime contain matter: all vacuum solutions to the Einstein field equations representing an isolated massive object necessarily possess regions of strong curvature. Such strong-field regions would affect the Green's function in a way that cannot be predicted by a perturbative calculation; the entire method would fail, even if the Green's function were to be evaluated only in the weak-field region of the spacetime. Thus, the global character of the Green's function, together with the weak-field limitations of our calculational methods, dictate that we work with spacetimes that contain matter.

The problem resides with the fact that the equations of motion for a massive particle, as given by Eqs. (1.2) and (1.3), are restricted to vacuum spacetimes. The Mino-Sasaki-Tanaka-Quinn-Wald equations of motion [26.27] are therefore not directly suited to a weak-field calculation. An extension to spacetimes containing matter must be produced. We do this in Sec. VI, proceeding in two steps.

First, we incorporate the modifications to the gravitational Green's function that come from the presence of Riccitensor terms in Green's equation. These modifications affect the gravitational self-force, but they do not alter Eq. (1.3) if the particle is restricted to move in a vacuum region of the spacetime. Second, we consider how the presence of matter modifies the equations of motion. Because the background stress-energy tensor $T^{\alpha \beta}$ depends on the metric, it necessarily suffers a perturbation when the massive particle is inserted in the spacetime. Physically, this corresponds to the fact that while the object of mass $M$ - the star - is at rest when the particle is absent, it must move in the presence of the particle: both objects move around a fixed center of mass [50]. This perturbative motion of the star produces an additional metric perturbation, over and above the perturbation directly associated with the particle's own stress-energy tensor. This must be accounted for, and we shall see that its effect is to modify the right-hand side of Eq. (1.2) by a term $f_{\mathrm{mm}}^{\alpha}$ which we call the "matter-mediated" force. While the self-force can be thought of as a direct action of the particle on itself, this additional force can be thought of as an indirect action mediated by the presence of matter: the particle acts on the star, and the star acts back on the particle.

The gravitational self-force is computed in Sec. V, and this calculation incorporates the effects of the matter on the retarded Green's function. We obtain

$$
\boldsymbol{f}_{\text {grav }}=-\frac{11}{3} m^{2} \frac{d \boldsymbol{g}}{d t}
$$

and according to the naive equations of motion $m d^{2} \boldsymbol{z} / d t^{2}=m \boldsymbol{g}+\boldsymbol{f}_{\text {grav }}$, the gravitational self-force does work on the particle at an average rate $d W / d t=+\frac{11}{3} m^{2}|\boldsymbol{g}|^{2}$; it therefore gives rise to radiation antidamping [51]. Notice that the self-force represents a correction of 1.5PN (post-Newtonian) order to the Newtonian equations of motion 52]. Notice also that the dual point of view seems at work also here: our expression for the "radiation-reaction force" could be 
calculated on the basis of Eq. (1.2) by adopting a "flat-spacetime point of view" in which $f_{\text {grav }}^{\alpha}$ would be set to zero and the "external force" equated to $m \boldsymbol{g}$.

The remarkable conspiracy that makes the tail integral reproduce the external-force term in the equations of motion is seen to be at play in all three cases. While the agreement between the flat-spacetime and curved-spacetime points of view is quite necessary in the case of the scalar and electromagnetic self-forces, it is decidedly disturbing in the gravitational case. How can we understand this result?

The answer obviously comes from the matter-mediated force, which we define and compute in Sec. VI. For this we obtain

$$
\boldsymbol{f}_{\mathrm{mm}}=m \delta \boldsymbol{g}+1 \mathrm{PN}+\frac{11}{3} m^{2} \frac{d \boldsymbol{g}}{d t},
$$

where the first term represents the change in the star's Newtonian gravitational field associated with its motion around the fixed center of mass, the second term is a post-Newtonian correction to the Newtonian force $m \boldsymbol{g}$, and the third term is a radiation-damping term that precisely cancels out the antidamping force of Eq. (1.13). (Such a cancellation was noticed a long time ago by Carmeli [53], in the context of a very different formulation of the equations of motion.)

A precise expression for the matter-mediated force can be found in Eq. (6.54) below. When it is substituted, together with the self-force, into the equations of motion, we find that they take the form

$$
\frac{d^{2} \boldsymbol{z}}{d t^{2}}=-\frac{M}{\rho^{3}}\left[\left(1+\boldsymbol{v}^{2}-\frac{5 m}{\rho}-4 \boldsymbol{v} \cdot \boldsymbol{V}\right) \boldsymbol{\rho}-\boldsymbol{\rho} \cdot(4 \boldsymbol{v}-3 \boldsymbol{V}) \boldsymbol{v}+4(\boldsymbol{\rho} \cdot \boldsymbol{v}) \boldsymbol{V}\right]+O\left(2 \mathrm{PN}, m^{2}, M^{2}\right),
$$

which contains no trace of a radiation-reaction force. Here, $\boldsymbol{z}(t)$ gives the position of the particle, and $\boldsymbol{v}(t)=d \boldsymbol{z} / d t$ is its velocity; the star moves on a trajectory $\boldsymbol{Z}(t)$ with a velocity $\boldsymbol{V}(t)=d \boldsymbol{Z} / d t$. We use $\boldsymbol{\rho}=\boldsymbol{z}-\boldsymbol{Z}$ to designate the separation between the two objects, and $\rho \equiv|\boldsymbol{z}-\boldsymbol{Z}|$. The equations of motion for the star are

$$
\frac{d^{2} \boldsymbol{Z}}{d t^{2}}=\frac{m}{\rho^{3}}\left\{\left[1+2 \boldsymbol{v}^{2}-\frac{3}{2}\left(\frac{\boldsymbol{\rho} \cdot \boldsymbol{v}}{\rho}\right)^{2}\right] \boldsymbol{\rho}-3(\boldsymbol{\rho} \cdot \boldsymbol{v}) \boldsymbol{v}\right\}+O\left(2 \mathrm{PN}, m^{2}, m M\right) .
$$

Our calculations cannot reproduce all the terms in the Einstein-Infeld-Hoffmann equations of motion [1.54]: Eq. (1.15) omits terms that are quadratic in $m$ and $M$, while Eq. (1.16) neglects terms of order $m^{2}$ and $m M$. The formalism we use in this paper is based on linear perturbation theory, and it would be incapable of producing corrections of order $m^{2}$. On the other hand, the corrections of order $m M$ and $M^{2}$ are within the reach of the formalism, but in order to produce them we would need to go beyond the weak-field approximation considered in this paper. Within these limitations, however, we have complete agreement between the calculations presented in this paper and the standard post-Newtonian treatment of the two-body problem.

\section{Organization}

The technical part of the paper begins in Sec. II with a detailed discussion of the metric of Eq. (1.9), and a derivation of the scalar, electromagnetic, and gravitational wave equations for the weakly curved spacetimes described by this metric. In Sec. III we introduce the two-point functions $A\left(x, x^{\prime}\right)$ and $B\left(x, x^{\prime}\right)$, and show how the scalar, electromagnetic, and gravitational Green's functions can be obtained from them by acting with differential operators. In Sec. IV we introduce methods to compute the two-point functions, and evaluate them in interesting limiting cases. [In Appendix A we expand our discussion of $A\left(x, x^{\prime}\right)$, and in Appendix B we provide an explicit computation of the two-point functions for the special case $\Phi=-M / r$.] In Sec. V we use our preceding results for the Green's functions to compute the scalar, electromagnetic, and gravitational self-forces for the spacetimes of Eq. (1.9); our results were quoted in Eqs. (1.12), (1.10), and (1.13), respectively. Finally, in Sec. VI we introduce and compute the gravitational matter-mediated force, and show that it cancels out the antidamping force calculated in Sec. V.

\section{WEAKLY CURVED SPACETIME}

\section{A. The spacetime and its geometric quantities}

The spacetimes considered in this paper have a metric given by 


$$
d s^{2}=-(1+2 \Phi) d t^{2}+(1-2 \Phi)\left(d x^{2}+d y^{2}+d z^{2}\right),
$$

in which $\Phi(\boldsymbol{x})$ is a Newtonian potential, a function of the spatial coordinates $\boldsymbol{x}$ satisfying Poisson's equation,

$$
\nabla^{2} \Phi=4 \pi \rho,
$$

where $\rho(\boldsymbol{x})$ is the mass density. By virtue of Eq. (2.2) and the fact that $\Phi \ll 1$ everywhere in the spacetime, the metric of Eq. (2.1) satisfies the linearized Einstein field equations. Throughout the paper we will work consistently to first order in $\Phi$. If the metric were representing the gravitational field of a point mass $M$ located at the origin of the coordinate system, then $\rho=M \delta(\boldsymbol{x})$ and $\Phi=-M / r$, where $r=|\boldsymbol{x}|$. We will not need to adopt this particular form for the Newtonian potential (which would violate the condition that $\Phi$ be small everywhere), and we shall work with generic potentials satisfying Eq. (2.2). We will, however, assume that $\Phi$ becomes equal to $-M / r$, with $M=\int \rho d^{3} x$, far from the matter distribution. Notice that we do not allow $\Phi$ to depend on time.

Introducing $t^{\alpha}=\partial x^{\alpha} / \partial t$ as the timelike Killing vector of the spacetime, we write the metric tensor as

$$
g_{\alpha \beta}=\eta_{\alpha \beta}-2 \Phi \chi_{\alpha \beta}, \quad \chi_{\alpha \beta} \equiv \eta_{\alpha \beta}+2 t_{\alpha} t_{\beta},
$$

where $\eta_{\alpha \beta}=\operatorname{diag}(-1,1,1,1)$ is the Minkowski metric, which was used to lower the index on $t^{\alpha}$. Throughout this section we will continue to lower and raise indices with the Minkowski metric, unless it is otherwise indicated. Notice that $\chi_{\alpha \beta}=\operatorname{diag}(1,1,1,1)$. From Eq. (2.3) it is easy to derive $\sqrt{-g}=1-2 \Phi$ and $g^{\alpha \beta}=\eta^{\alpha \beta}+2 \Phi \chi^{\alpha \beta}$. These results hold to first order in $\Phi$.

The metric of Eq. (2.3) comes with the following Christoffel symbols:

$$
\Gamma_{\alpha \beta}^{\mu}=-\chi_{\alpha}^{\mu} \Phi_{, \beta}-\chi_{\beta}^{\mu} \Phi_{, \alpha}+\chi_{\alpha \beta} \Phi^{, \mu} .
$$

As a consequence of the relation $\Phi_{, \alpha} t^{\alpha}=0$, they satisfy $\eta^{\alpha \beta} \Gamma_{\alpha \beta}^{\mu}=0$. The Riemann tensor is given by

$$
R_{\alpha \beta \gamma}^{\mu}=-\chi_{\gamma}^{\mu} \Phi_{, \alpha \beta}+\chi_{\beta}^{\mu} \Phi_{, \alpha \gamma}-\chi_{\alpha \beta} \Phi_{\gamma}^{, \mu}+\chi_{\alpha \gamma} \Phi_{\beta}^{, \mu} .
$$

Contracting over the first and third indices gives the Ricci tensor:

$$
R_{\alpha \beta}=\chi_{\alpha \beta} \square \Phi,
$$

where $\square \Phi \equiv \eta^{\alpha \beta} \Phi_{, \alpha \beta}$. Because $\Phi$ does not depend on time, $\square \Phi=\nabla^{2} \Phi \equiv \delta^{a b} \Phi_{, a b}$, where $x^{a}$ denotes the spatial coordinates. The Ricci scalar is $R=2 \square \Phi$, and the Einstein tensor is $G^{\alpha \beta}=2 t^{\alpha} t^{\dot{\beta}} \square \Phi$. Equation (2.2) follows from the Einstein field equations with $T^{\alpha \beta}=\rho t^{\alpha} t^{\beta}$; this represents a fluid of mass density $\rho$ and negligible pressure at rest in the spacetime.

\section{B. Wave equations}

The field equation for a massless scalar field $\psi$ in a curved spacetime with metric $g_{\alpha \beta}$ is

$$
g^{\alpha \beta} \psi_{; \alpha \beta}-\xi R \psi=-4 \pi \mu,
$$

where the semicolon designates covariant differentiation compatible with the metric tensor, $\xi$ is an arbitrary constant measuring the coupling to curvature, and $\mu$ is a given source term. It will be convenient to work with a densitized form for the field equation,

$$
E[\psi]=-4 \pi \sqrt{-g} \mu,
$$

where $E[\psi]$ stands for the left-hand side of Eq. (2.7) multiplied by $\sqrt{-g}$. For the weakly curved spacetime of Eq. (2.3), we find that

$$
E[\psi]=\square \psi+4 \Phi t^{\alpha} t^{\beta} \psi_{, \alpha \beta}-2 \xi(\square \Phi) \psi,
$$

where $\square \equiv \eta^{\alpha \beta} \partial_{\alpha} \partial_{\beta}$ is the flat-spacetime wave operator and, as was pointed out previously, $\square \Phi=\nabla^{2} \Phi=4 \pi \rho$.

An electromagnetic field is represented by a vector potential $A^{\alpha}$ which is here assumed to satisfy the Lorentz-gauge condition, $A_{; \alpha}^{\alpha}=0$. Its field equations are

$$
g^{\mu \nu} A_{; \mu \nu}^{\alpha}-R_{\mu}^{\alpha} A^{\mu}=-4 \pi j^{\alpha},
$$


where $j^{\alpha}$ is a given current density. The wave equation in densitized form is

$$
E^{\alpha}[A]=-4 \pi \sqrt{-g} j^{\alpha},
$$

where $E^{\alpha}[A]$ stands for the left-hand side of Eq. (2.10) multiplied by $\sqrt{-g}$. A straightforward computation reveals that for the weakly curved spacetime of Eq. 2.3),

$$
E^{\alpha}[A]=\square A^{\alpha}+4 \Phi t^{\mu} t^{\nu} A_{, \mu \nu}^{\alpha}-2\left(\chi_{\mu}^{\alpha} \Phi_{, \nu}+\chi_{\nu}^{\alpha} \Phi_{, \mu}-\chi_{\mu \nu} \Phi^{, \alpha}\right) A^{\mu, \nu}-\left[\chi^{\alpha \mu} \Phi_{, \mu \nu}+2 \chi_{\nu}^{\alpha} \square \Phi-\chi_{\nu}^{\mu} \Phi_{\mu}^{, \alpha}\right] A^{\nu} .
$$

Here, $\square$ acting on the vector field $A^{\alpha}$ still stands for the scalar wave operator of flat spacetime.

A gravitational perturbation on a background metric $g_{\alpha \beta}$ is described by a tensor $\gamma_{\alpha \beta}$, and the full metric is $\hat{g}_{\alpha \beta}=g_{\alpha \beta}+\gamma_{\alpha \beta}$. By linearizing the Einstein field equations about the background, one obtains a wave equation for the perturbation. This equation takes a simpler form if it is expressed in terms of a "trace-reversed" field $\bar{\gamma}_{\alpha \beta} \equiv \gamma_{\alpha \beta}-\frac{1}{2}\left(g^{\mu \nu} \gamma_{\mu \nu}\right) g_{\alpha \beta}$, which is then chosen to satisfy a Lorentz-gauge condition: $\bar{\gamma}_{; \beta}^{\alpha \beta}=0$. (Here and below, indices on the perturbation field are raised and lowered with the background metric, and a semicolon designates covariant differentiation on the background.) With these choices, the Einstein field equations take the form of a wave equation for $\bar{\gamma}^{\alpha \beta}$ [28]:

$$
g^{\mu \nu} \bar{\gamma}_{; \mu \nu}^{\alpha \beta}+2 R_{\mu \nu}^{\alpha \beta} \bar{\gamma}^{\mu \nu}+S_{\mu \nu}^{\alpha \beta} \bar{\gamma}^{\mu \nu}=-16 \pi \delta T^{\alpha \beta},
$$

where $\delta T^{\alpha \beta}$ is the perturbation in the stress-energy tensor (so that the full stress-energy tensor is $\hat{T}^{\alpha \beta}=T^{\alpha \beta}+\delta T^{\alpha \beta}$, with the first term denoting the background's stress-energy tensor), $R_{\mu \alpha \nu \beta}$ is the Riemann tensor of the background spacetime, and $S_{\mu \alpha \nu \beta}$ is constructed from the background's Ricci and Einstein tensors,

$$
S_{\mu \alpha \nu \beta}=2 R_{\mu(\alpha} g_{\beta) \nu}-R_{\mu \nu} g_{\alpha \beta}-R g_{\mu(\alpha} g_{\beta) \nu}-2 g_{\mu \nu} G_{\alpha \beta} .
$$

The parentheses around tensor indices indicate symmetrization with respect to these indices. Once Eq. (2.13) has been solved for the trace-reversed perturbation, the metric is recovered from the relation $\gamma_{\alpha \beta}=\bar{\gamma}_{\alpha \beta}-\frac{1}{2} g_{\alpha \beta} \bar{\gamma}$, where $\bar{\gamma}=g^{\alpha \beta} \bar{\gamma}_{\alpha \beta}$.

The densitized wave equation is

$$
E^{\alpha \beta}[\bar{\gamma}]=-16 \pi \sqrt{-g} \delta T^{\alpha \beta},
$$

where $E^{\alpha \beta}[\bar{\gamma}]$ stands for the left-hand side of Eq. (2.13) multiplied by $\sqrt{-g}$. A rather long but straightforward computation reveals that in a weakly curved spacetime with metric (2.3),

$$
\begin{aligned}
E^{\alpha \beta}[\bar{\gamma}]= & \square \bar{\gamma}^{\alpha \beta}+4 \Phi t^{\mu} t^{\nu} \bar{\gamma}_{, \mu \nu}^{\alpha \beta}-4\left(\chi_{\mu}^{(\alpha} \Phi_{, \nu}+\chi_{\nu}^{(\alpha} \Phi_{, \mu}-\chi_{\mu \nu} \Phi^{(, \alpha}\right) \bar{\gamma}^{\beta) \mu, \nu}+2\left(\chi_{\mu \nu} \Phi^{, \alpha \beta}-2 \chi_{\mu}^{(\alpha} \Phi_{\nu}^{, \beta)}+\chi^{\alpha \beta} \Phi_{, \mu \nu}\right) \bar{\gamma}^{\mu \nu} \\
& -\square \Phi\left(\eta^{\alpha \beta} \chi_{\mu \nu} \bar{\gamma}^{\mu \nu}+2 \bar{\gamma}^{\alpha \beta}+4 t^{\alpha} t^{\beta} \eta_{\mu \nu} \bar{\gamma}^{\mu \nu}\right) .
\end{aligned}
$$

In the first term on the right-hand side, $\square=\eta^{\mu \nu} \partial_{\mu} \partial_{\nu}$ is the scalar wave operator.

\section{GREEN'S FUNCTIONS}

\section{A. Generating two-point functions}

The task before us in this section is the computation of retarded Green's functions for the wave equations considered in Sec. II - Eqs. (2.8), (2.11), and (2.15). As we shall see, these Green's functions can be constructed by acting with differential operators on two generating two-point functions, $A\left(x, x^{\prime}\right)$ and $B\left(x, x^{\prime}\right)$, defined by

$$
A\left(x, x^{\prime}\right)=\frac{1}{2 \pi} \int G^{\mathrm{flat}}\left(x, x^{\prime \prime}\right) \Phi\left(x^{\prime \prime}\right) G^{\mathrm{flat}}\left(x^{\prime \prime}, x^{\prime}\right) d^{4} x^{\prime \prime}
$$

and

$$
B\left(x, x^{\prime}\right)=\int G^{\mathrm{flat}}\left(x, x^{\prime \prime}\right) \rho\left(x^{\prime \prime}\right) G^{\mathrm{flat}}\left(x^{\prime \prime}, x^{\prime}\right) d^{4} x^{\prime \prime} .
$$

Here, $\Phi$ is the Newtonian potential of Eq. (2.1), $\rho$ is the mass density of Eq. (2.2), and $G^{\text {flat }}\left(x, x^{\prime}\right)$ is the retarded Green's function of the flat-spacetime wave operator, 


$$
G^{\mathrm{flat}}\left(x, x^{\prime}\right)=\frac{\delta\left(t-t^{\prime}-\left|\boldsymbol{x}-\boldsymbol{x}^{\prime}\right|\right)}{\left|\boldsymbol{x}-\boldsymbol{x}^{\prime}\right|}
$$

this satisfies $\square G^{\text {flat }}\left(x, x^{\prime}\right)=-4 \pi \delta_{4}\left(x-x^{\prime}\right)$, where $\delta_{4}\left(x-x^{\prime}\right)$ is a four-dimensional $\delta$-function, equal to $\delta\left(t-t^{\prime}\right) \delta_{3}\left(\boldsymbol{x}-\boldsymbol{x}^{\prime}\right)$. This method to calculate the Green's function originated in the work of DeWitt and DeWitt [48], who used it to compute the scalar and electromagnetic Green's functions for the special case $\Phi=-M / r$. Here we generalize their method to arbitrary potentials, and to the gravitational Green's function.

Inside the integrals of Eqs. (3.1) and (3.2), the function $G^{\text {flat }}\left(x, x^{\prime \prime}\right)$ picks out the past light cone of the point $x$, while $G^{\text {flat }}\left(x^{\prime \prime}, x^{\prime}\right)$ picks out the future light cone of the point $x^{\prime}$. (These light cones are those of the flat background spacetime.) The intersection of the two light cones defines a closed two-surface $\mathcal{S}$, and we see that $A\left(x, x^{\prime}\right)$ is the average of the Newtonian potential over $\mathcal{S}$, while $B\left(x, x^{\prime}\right)$ is the average of the mass density. Both two-point functions are zero if $x$ and $x^{\prime}$ are spacelike related, because the surface of intersection then disappears.

Averages of derivatives of the Newtonian potential can be obtained by differentiating $A\left(x, x^{\prime}\right)$. For example,

$$
\frac{1}{2 \pi} \int G^{\mathrm{flat}}\left(x, x^{\prime \prime}\right)\left[\partial_{\alpha^{\prime \prime}} \Phi\left(x^{\prime \prime}\right)\right] G^{\mathrm{fat}}\left(x^{\prime \prime}, x^{\prime}\right) d^{4} x^{\prime \prime}=\left(\partial_{\alpha}+\partial_{\alpha^{\prime}}\right) A\left(x, x^{\prime}\right)
$$

and

$$
\frac{1}{2 \pi} \int G^{\mathrm{flat}}\left(x, x^{\prime \prime}\right)\left[\partial_{\alpha^{\prime \prime} \beta^{\prime \prime}} \Phi\left(x^{\prime \prime}\right)\right] G^{\mathrm{fat}}\left(x^{\prime \prime}, x^{\prime}\right) d^{4} x^{\prime \prime}=\left(\partial_{\alpha \beta}+\partial_{\alpha^{\prime} \beta}+\partial_{\alpha \beta^{\prime}}+\partial_{\alpha^{\prime} \beta^{\prime}}\right) A\left(x, x^{\prime}\right) .
$$

We use the notation $\partial_{\alpha^{\prime}} f=\partial f / \partial x^{\alpha^{\prime}}, \partial_{\alpha \beta^{\prime}} f=\partial^{2} f / \partial x^{\alpha} \partial x^{\beta^{\prime}}$, etc., in which the tensor index (either $\alpha, \alpha^{\prime}$, or $\alpha^{\prime \prime}$ ) indicates with respect to which variable (between $x, x^{\prime}$, and $x^{\prime \prime}$ ) the function $f$ is being differentiated.

Equations (3.4) and (3.5) are easy to establish. (In the following we keep the notation simple and drop the factor of $1 / 2 \pi$ in front of the integrals). First we write $\partial_{\alpha} A=\int\left(\partial_{\alpha} G\right) \Phi G d x^{\prime \prime}$, and use the fact that $G^{\text {flat }}\left(x, x^{\prime \prime}\right)$ depends only on the difference between $x$ and $x^{\prime \prime}$. This allows us to express the right-hand side as - $\int\left(\partial_{\alpha^{\prime \prime}} G\right) \Phi G d x^{\prime \prime}$. Integrating by parts, we obtain $+\int G\left(\partial_{\alpha^{\prime \prime}} \Phi\right) G d x^{\prime \prime}+\int G \Phi\left(\partial_{\alpha^{\prime \prime}} G\right) d x^{\prime \prime}$. In the second term, the derivative operator can be switched to $-\partial_{\alpha^{\prime}}$, which can be taken outside the integral. The second term becomes $-\partial_{\alpha^{\prime}} A$, and Eq. (3.4) follows. To derive Eq. (3.5) we differentiate Eq. (3.4) with respect to $x^{\beta}$, and go through the same procedure.

Because the Newtonian potential does not depend on time, Eq. (3.4) implies

$$
\partial_{t} A\left(x, x^{\prime}\right)+\partial_{t^{\prime}} A\left(x, x^{\prime}\right)=0 .
$$

Another useful identity results from taking the trace of Eq. (3.5). Using Poisson's equation $\square \Phi=\nabla^{2} \Phi=4 \pi \rho$ and Eq. (3.2), we obtain

$$
\frac{1}{2}\left(\square+\square^{\prime}\right) A\left(x, x^{\prime}\right)+\partial_{\alpha}^{\alpha^{\prime}} A\left(x, x^{\prime}\right)=B\left(x, x^{\prime}\right),
$$

where $\square^{\prime} \equiv \eta^{\mu \nu} \partial_{\mu^{\prime}} \partial_{\nu^{\prime}}$; summation over $\alpha^{\prime}$ and $\alpha$ in the third term is understood. By virtue of Eq. (3.6), all time derivatives drop out of Eq. (3.7), which can then be written as $\frac{1}{2}\left(\nabla^{2}+\nabla^{\prime 2}\right) A\left(x, x^{\prime}\right)+\partial_{a}^{a^{\prime}} A\left(x, x^{\prime}\right)=B\left(x, x^{\prime}\right)$. The left-hand side of Eq. (3.7) can be simplified differently. The wave operator acting on $A$ can be taken inside the integral, where it acts on $G^{\text {flat }}$. This returns a $\delta$-function, and the integral can be evaluated. This gives

$$
\square A\left(x, x^{\prime}\right)=-2 \Phi(x) G^{\mathrm{flat}}\left(x, x^{\prime}\right), \quad \square^{\prime} A\left(x, x^{\prime}\right)=-2 \Phi\left(x^{\prime}\right) G^{\mathrm{flat}}\left(x, x^{\prime}\right),
$$

and these expressions can be substituted into Eq. (3.7).

\section{B. Scalar Green's function}

We are looking for a function $G\left(x, x^{\prime}\right)$ that solves 41]

$$
E[G]=-4 \pi \delta_{4}\left(x-x^{\prime}\right)
$$

to first order in the Newtonian potential $\Phi$. Here, $\delta_{4}\left(x-x^{\prime}\right)$ is the four-dimensional $\delta$-function, and $E[G]$ stands for the left-hand side of Eq. (2.7) multiplied by $\sqrt{-g}$, with $G\left(x, x^{\prime}\right)$ taking the place of $\psi(x)$. In terms of this Green's function, the solution to Eq. (2.8) is 


$$
\psi(x)=\int G\left(x, x^{\prime}\right) \mu\left(x^{\prime}\right) \sqrt{-g^{\prime}} d^{4} x^{\prime},
$$

where $g^{\prime}$ is the metric determinant evaluated at $x^{\prime}$.

To find this Green's function, we write

$$
G\left(x, x^{\prime}\right)=G^{\mathrm{flat}}\left(x, x^{\prime}\right)+\dot{G}\left(x, x^{\prime}\right)+O\left(\Phi^{2}\right),
$$

in which $G^{\text {flat }}\left(x, x^{\prime}\right)$ is flat-spacetime solution given by Eq. (3.3), and $\dot{G}\left(x, x^{\prime}\right)$ is the first-order correction (linear in the Newtonian potential). [The overdot notation suggests that $G\left(x, x^{\prime}\right)$ should be viewed as a smooth function of a smallness parameter $\epsilon$, and that Eq. (3.11) gives the first two terms of its Taylor expansion about $\epsilon=0$.] By virtue of Eq. (2.9), in which we substitute $\square \Phi=4 \pi \rho$, we find that $\dot{G}\left(x, x^{\prime}\right)$ satisfies

$$
\square \dot{G}\left(x, x^{\prime}\right)=-4 \Phi(x) \partial_{t t} G^{\mathrm{flat}}\left(x, x^{\prime}\right)+8 \pi \xi \rho(x) G^{\mathrm{flat}}\left(x, x^{\prime}\right) .
$$

The solution is

$$
\dot{G}\left(x, x^{\prime}\right)=\frac{1}{\pi} \int G^{\mathrm{flat}}\left(x, x^{\prime \prime}\right) \Phi\left(x^{\prime \prime}\right) \partial_{t^{\prime \prime} t^{\prime \prime}} G^{\mathrm{flat}}\left(x^{\prime \prime}, x^{\prime}\right) d^{4} x^{\prime \prime}-2 \xi \int G^{\mathrm{flat}}\left(x, x^{\prime \prime}\right) \rho\left(x^{\prime \prime}\right) G^{\mathrm{flat}}\left(x^{\prime \prime}, x^{\prime}\right) d^{4} x^{\prime \prime} .
$$

In the first integral, the derivative operator can be switched to $\partial_{t^{\prime} t^{\prime}}$ because the flat-spacetime Green's function depends only on $x^{\prime \prime}-x^{\prime}$. The derivatives can then be taken outside the integral. Using Eqs. (3.1) and (3.2), we obtain $\dot{G}=2 \partial_{t^{\prime} t^{\prime}} A-2 \xi B$. Using Eq. (3.6), we express the final result as

$$
\dot{G}\left(x, x^{\prime}\right)=-2 \partial_{t t^{\prime}} A\left(x, x^{\prime}\right)-2 \xi B\left(x, x^{\prime}\right) .
$$

Equations (3.11) and (3.14) give the scalar Green's function to first order in $\Phi$. As was claimed previously, $G\left(x, x^{\prime}\right)$ can be expressed in terms of the generating two-point functions introduced in Sec. III A. For the special case $\xi=0$, our Green's function agrees with the one obtained by DeWitt and DeWitt [48].

\section{Electromagnetic Green's function}

The electromagnetic Green's function $G_{\beta^{\prime}}^{\alpha}\left(x, x^{\prime}\right)$ is a solution to 41

$$
E_{\beta^{\prime}}^{\alpha}[G]=-4 \pi \delta_{\beta^{\prime}}^{\alpha} \delta_{4}\left(x-x^{\prime}\right)
$$

where $E_{\beta^{\prime}}^{\alpha}[G]$ stands for the left-hand side of Eq. (2.10) multiplied by $\sqrt{-g}$, with $G_{\beta^{\prime}}^{\alpha}\left(x, x^{\prime}\right)$ taking the place of $A^{\alpha}(x)$. Here and below, the unprimed tensor indices refer to the point $x$, while the primed indices refer to $x^{\prime}$. In terms of this Green's function, the solution to Eq. (2.11) is

$$
A^{\alpha}(x)=\int G_{\beta^{\prime}}^{\alpha}\left(x, x^{\prime}\right) j^{\beta^{\prime}}\left(x^{\prime}\right) \sqrt{-g^{\prime}} d^{4} x^{\prime} .
$$

We seek to determine the Green's function to first order in $\Phi$.

For this purpose, we write

$$
G_{\beta^{\prime}}^{\alpha}\left(x, x^{\prime}\right)=G^{\mathrm{flat}}\left(x, x^{\prime}\right) \delta_{\beta^{\prime}}^{\alpha}+\dot{G}_{\beta^{\prime}}^{\alpha}\left(x, x^{\prime}\right)+O\left(\Phi^{2}\right)
$$

and substitute into Eq. (3.15). Here, $G^{\text {flat }}\left(x, x^{\prime}\right)$ is the scalar Green's function of Eq. (3.3), and $\dot{G}_{\beta^{\prime}}^{\alpha}\left(x, x^{\prime}\right)$ is the first-order correction to the electromagnetic Green's function. We find that this must satisfy

$$
\square \dot{G}_{\beta^{\prime}}^{\alpha}=-4 \Phi \partial_{t t} G^{\mathrm{flat}} \delta_{\beta}^{\alpha}+2\left(\chi_{\mu}^{\alpha} \Phi_{, \beta}+\chi_{\beta}^{\alpha} \Phi_{, \mu}-\chi_{\beta \mu} \Phi^{, \alpha}\right) G^{\mathrm{flat}, \mu}+8 \pi \rho \chi_{\beta}^{\alpha} G^{\mathrm{flat}} .
$$

We have used the fact that $\Phi$ does not depend on time, so that, for example, $\chi^{\alpha \mu} \Phi_{, \mu \beta}=\eta^{\alpha \mu} \Phi_{, \mu \beta}=\Phi_{\beta}^{, \alpha}$; such terms end up canceling out. We have also, on the right-hand side, dropped the distinction between $\beta$ and $\beta^{\prime}$; this distinction is not necessary when dealing with a constant tensor such as $\chi_{\beta}^{\alpha}$.

Equation (3.18) is solved by once again invoking the flat-spacetime Green's function. The solution is

$$
\dot{G}_{\beta^{\prime}}^{\alpha}\left(x, x^{\prime}\right)=-\frac{1}{4 \pi} \int G^{\mathrm{fat}}\left(x, x^{\prime \prime}\right) \operatorname{RHS}\left(x^{\prime \prime}\right) d^{4} x^{\prime \prime},
$$


where $\operatorname{RHS}\left(x^{\prime \prime}\right)$ stands for the right-hand side of Eq. (3.18) evaluated at $x=x^{\prime \prime}$. The derivatives appearing in Eq. (3.18) are now taken with respect to $x^{\prime \prime}$. When $\partial_{\mu^{\prime \prime}}$ (say) is acting on $G^{\text {flat }}\left(x^{\prime \prime}, x^{\prime}\right)$, it can be replaced by $-\partial_{\mu^{\prime}}$ and taken outside the integral. This leaves us with integrals involving $G^{\text {flat }}\left(x, x^{\prime \prime}\right), G^{\text {flat }}\left(x^{\prime \prime}, x^{\prime}\right)$, as well as $\Phi\left(x^{\prime \prime}\right)$ and its derivatives. Those integrals can all be expressed in terms of the two-point functions $A\left(x, x^{\prime}\right)$ and $B\left(x, x^{\prime}\right)$ defined in Eqs. (3.1) and (3.2), thanks to the relations (3.4) and (3.5). The results can then be simplified by using Eqs. (3.6)-(3.8), as well as Eq. (2.3) for $\chi_{\alpha \beta}$. The end result is

$$
\dot{G}_{\beta^{\prime}}^{\alpha}=-2 \partial_{t t^{\prime}} A \delta_{\beta}^{\alpha}+\left(\partial_{\beta}^{\alpha^{\prime}}-\partial_{\beta^{\prime}}^{\alpha}\right) A+2 t^{\alpha}\left(\partial_{t^{\prime} \beta}-\partial_{t \beta^{\prime}}\right) A+2 t_{\beta}\left(\partial_{t}^{\alpha^{\prime}}-\partial_{t^{\prime}}^{\alpha}\right) A+\chi_{\beta}^{\alpha}\left(\Delta \Phi G^{\text {flat }}-B\right),
$$

where $\Delta \Phi \equiv \Phi(x)-\Phi\left(x^{\prime}\right)$. Equations (3.17) and (3.20) give $G_{\beta^{\prime}}^{\alpha}\left(x, x^{\prime}\right)$, the electromagnetic Green's function, to first order in $\Phi$.

Equation (3.20) is easier to deal with if we distinguish between its temporal and spatial components. From it we read off

$$
\begin{aligned}
& \dot{G}_{t^{\prime}}^{t}=-\Delta \Phi G^{\text {flat }}-2 \partial_{t t^{\prime}} A+B, \\
& \dot{G}_{a^{\prime}}^{t}=\left(\partial_{t^{\prime} a}-\partial_{t a^{\prime}}\right) A, \\
& \dot{G}_{t^{\prime}}^{a}=\left(\partial_{t^{\prime}}^{a}-\partial_{t}^{a^{\prime}}\right) A, \\
& \dot{G}_{b^{\prime}}^{a}=\delta_{b}^{a}\left(\Delta \Phi G^{\text {flat }}-2 \partial_{t t^{\prime}} A-B\right)+\left(\partial_{b}^{a^{\prime}}-\partial_{b^{\prime}}^{a}\right) A .
\end{aligned}
$$

We notice that in Eqs. (3.20) and (3.21), the part of $\dot{G}_{\beta^{\prime}}^{\alpha}\left(x, x^{\prime}\right)$ that involves $G^{\text {flat }}\left(x, x^{\prime}\right)$ has support only on the past light cone of the point $x$. The remaining part, that involving the two-point functions $A\left(x, x^{\prime}\right)$ and $B\left(x, x^{\prime}\right)$, have support inside the light cone as well; this is the "tail part" of the Green's function [29]. Our electromagnetic Green's function agrees with DeWitt and DeWitt [48].

\section{Gravitational Green's function}

The trace-reversed gravitational Green's function $\mathcal{G}^{\alpha \beta}{ }_{\gamma^{\prime} \delta^{\prime}}\left(x, x^{\prime}\right)$ satisfies the equation 28]

$$
E_{\gamma^{\prime} \delta^{\prime}}^{\alpha \beta}[\mathcal{G}]=-4 \pi \delta_{\gamma^{\prime}}^{(\alpha} \delta_{\delta^{\prime}}^{\beta)} \delta_{4}\left(x-x^{\prime}\right),
$$

where $E_{\gamma^{\prime} \delta^{\prime}}^{\alpha \beta}[\mathcal{G}]$ stands for the left-hand side of Eq. (2.13) multiplied by $\sqrt{-g}$, but with $\mathcal{G}_{\gamma^{\prime} \delta^{\prime}}^{\alpha \beta}\left(x, x^{\prime}\right)$ replacing $\bar{\gamma}^{\alpha \beta}(x)$. In terms of this Green's function, the solution to Eq. (2.15) is

$$
\bar{\gamma}^{\alpha \beta}(x)=4 \int \mathcal{G}_{\gamma^{\prime} \delta^{\prime}}^{\alpha \beta}\left(x, x^{\prime}\right) \delta T^{\gamma^{\prime} \delta^{\prime}}\left(x^{\prime}\right) \sqrt{-g^{\prime}} d^{4} x^{\prime} .
$$

Our goal here is again to calculate the Green's function to first order in the Newtonian potential. The steps are virtually identical to what was done for the scalar and electromagnetic cases. The only difference is that the expressions are longer.

At the end of the calculation we will have to "trace-reverse" the trace-reversed Green's function to obtain $G_{\gamma^{\prime} \delta^{\prime}}^{\alpha \beta}\left(x, x^{\prime}\right)$, the Green's function directly associated with the metric perturbation $\gamma_{\alpha \beta}$. This is given by

$$
G_{\gamma^{\prime} \delta^{\prime}}^{\alpha \beta}\left(x, x^{\prime}\right)=\mathcal{G}_{\gamma^{\prime} \delta^{\prime}}^{\alpha \beta}\left(x, x^{\prime}\right)-\frac{1}{2} g^{\alpha \beta}(x) g_{\mu \nu}(x) \mathcal{G}_{\gamma^{\prime} \delta^{\prime}}^{\mu \nu}\left(x, x^{\prime}\right) .
$$

In terms of this, the metric perturbation is recovered by integrating

$$
\gamma^{\alpha \beta}(x)=4 \int G_{\gamma^{\prime} \delta^{\prime}}^{\alpha \beta}\left(x, x^{\prime}\right) \delta T^{\gamma^{\prime} \delta^{\prime}}\left(x^{\prime}\right) \sqrt{-g^{\prime}} d^{4} x^{\prime} .
$$

To find the trace-reversed Green's function, we write

$$
\mathcal{G}_{\gamma^{\prime} \delta^{\prime}}^{\alpha \beta}\left(x, x^{\prime}\right)=G^{\mathrm{flat}}\left(x, x^{\prime}\right) \delta_{\gamma^{\prime}}^{(\alpha} \delta_{\delta^{\prime}}^{\beta)}+\dot{\mathcal{G}}_{\gamma^{\prime} \delta^{\prime}}^{\alpha \beta}\left(x, x^{\prime}\right)+O\left(\Phi^{2}\right),
$$

where $G^{\text {flat }}\left(x, x^{\prime}\right)$ is the flat-spacetime scalar Green's function, and $\dot{\mathcal{G}}_{\gamma^{\prime} \delta^{\prime}}\left(x, x^{\prime}\right)$ the correction of order $\Phi$. By substituting Eq. (3.26) into Eq. (3.22), we find that this must satisfy 


$$
\begin{aligned}
\square \dot{\mathcal{G}}_{\gamma^{\prime} \delta^{\prime}}^{\alpha \beta}= & -4 \Phi \partial_{t t} G^{\mathrm{flat}} \delta_{\gamma}^{(\alpha} \delta_{\delta}^{\beta)}+4 \delta_{(\gamma}^{(\alpha}\left(\chi_{\delta)}^{\beta)} \Phi_{, \mu}+\Phi_{, \delta)} \chi_{\mu}^{\beta)}-\chi_{\delta) \mu} \Phi^{, \beta)}\right) G^{\mathrm{flat}, \mu} \\
& -2\left(\chi_{\gamma \delta} \Phi^{, \alpha \beta}-2 \chi_{(\gamma}^{(\alpha} \Phi_{\delta)}^{, \beta)}+\chi^{\alpha \beta} \Phi_{, \gamma \delta}\right) G^{\mathrm{flat}}+4 \pi\left(\eta^{\alpha \beta} \chi_{\gamma \delta}+2 \delta_{\gamma}^{(\alpha} \delta_{\delta}^{\beta)}+4 t^{\alpha} t^{\beta} \eta_{\gamma \delta}\right) \rho G^{\mathrm{flat}},
\end{aligned}
$$

where we have gone through the same simplification steps as in the electromagnetic case. The remaining steps are also similar: We integrate Eq. (3.27) with the flat-spacetime Green's function, and we simplify the resulting integrals as was described in the paragraph preceding Eq. (3.20). After a rather long computation, we obtain

$$
\begin{aligned}
\dot{\mathcal{G}}_{\gamma^{\prime} \delta^{\prime}}^{\alpha \beta}= & -2 \partial_{t t^{\prime}} A \delta_{\gamma}^{(\alpha} \delta_{\delta}^{\beta)}+2 \delta_{(\gamma}^{(\alpha}\left[\partial_{\delta)}^{\left.\beta^{\prime}\right)}-\partial_{\left.\delta^{\prime}\right)}^{\beta)}+2 t^{\beta)}\left(\partial_{\delta) t^{\prime}}-\partial_{\left.\delta^{\prime}\right) t}\right)-2 t_{\delta)}\left(\partial_{t^{\prime}}^{\beta)}-\partial_{t}^{\left.\beta^{\prime}\right)}\right)\right] A+\chi_{\gamma \delta}\left(\partial^{\alpha}+\partial^{\alpha^{\prime}}\right)\left(\partial^{\beta}+\partial^{\beta^{\prime}}\right) A \\
& -2 \chi_{(\gamma}^{(\alpha}\left(\partial^{\beta)}+\partial^{\left.\beta^{\prime}\right)}\right)\left(\partial_{\delta)}+\partial_{\left.\delta^{\prime}\right)}\right) A+\chi^{\alpha \beta}\left(\partial_{\gamma}+\partial_{\gamma^{\prime}}\right)\left(\partial_{\delta}+\partial_{\delta^{\prime}}\right) A+2 \chi_{(\gamma}^{(\alpha} \delta_{\delta)}^{\beta)} \Delta \Phi G^{\text {flat }} \\
& +\left(2 \chi_{(\gamma}^{(\alpha} \delta_{\delta)}^{\beta)}-\eta^{\alpha \beta} \chi_{\gamma \delta}-2 \delta_{\gamma}^{(\alpha} \delta_{\delta}^{\beta)}-4 t^{\alpha} t^{\beta} \eta_{\gamma \delta}\right) B,
\end{aligned}
$$

where $\Delta \Phi=\Phi(x)-\Phi\left(x^{\prime}\right)$. Equations (3.26) and (3.28) give $\mathcal{G}^{\alpha \beta}{ }_{\gamma^{\prime} \delta^{\prime}}\left(x, x^{\prime}\right)$, the trace-reversed gravitational Green's function, to first order in $\Phi$.

From Eq. (3.28) we extract the following components:

$$
\begin{aligned}
& \dot{\mathcal{G}}^{t t}{ }_{t^{\prime} t^{\prime}}=-2 \Delta \Phi G^{\text {flat }}-2 \partial_{t t^{\prime}} A+B, \\
& \dot{\mathcal{G}}^{t t}{ }_{t^{\prime} a^{\prime}}=\left(\partial_{t^{\prime} a}-\partial_{t a^{\prime}}\right) A \text {, } \\
& \dot{\mathcal{G}}^{t t}{ }_{a^{\prime} b^{\prime}}=\left(\partial_{a}+\partial_{a^{\prime}}\right)\left(\partial_{b}+\partial_{b^{\prime}}\right) A-3 \delta_{a b} B, \\
& \dot{\mathcal{G}}^{t a}{ }_{t^{\prime} t^{\prime}}=\left(\partial_{t^{\prime}}^{a}-\partial^{a^{\prime}}\right) A, \\
& \dot{\mathcal{G}}^{t a}{ }_{t^{\prime} b^{\prime}}=-\delta^{a}{ }_{b}\left(\partial_{t t^{\prime}} A+B\right)+\frac{1}{2}\left(\partial_{b}^{a}+2 \partial^{a^{\prime}}{ }_{b}+\partial_{b^{\prime}}^{a^{\prime}}\right) A \text {, } \\
& \dot{\mathcal{G}}^{t a}{ }_{b^{\prime} c^{\prime}}=\delta_{(b}^{a}\left(\partial_{c) t^{\prime}}-\partial_{\left.c^{\prime}\right) t}\right) A \text {, } \\
& \dot{\mathcal{G}}^{a b}{ }_{t^{\prime} t^{\prime}}=\left(\partial^{a}+\partial^{a^{\prime}}\right)\left(\partial^{b}+\partial^{b^{\prime}}\right) A-\delta^{a b} B, \\
& \dot{\mathcal{G}}^{a b}{ }_{t^{\prime} c^{\prime}}=\delta_{c}^{(a}\left(\partial^{b)}{ }_{t^{\prime}}-\partial^{\left.b^{\prime}\right)}{ }_{t}\right) A, \\
& \dot{\mathcal{G}}^{a b}{ }_{c^{\prime} d^{\prime}}=2 \delta_{c}^{(a} \delta^{b)}\left(\Delta \Phi G^{\mathrm{flat}}-\partial_{t t^{\prime}} A\right)+\delta^{a b}\left(\partial_{c}+\partial_{c^{\prime}}\right)\left(\partial_{d}+\partial_{d^{\prime}}\right) A-2 \delta_{(c}^{(a}\left(\partial_{d)}^{b)}+2 \partial_{\left.d^{\prime}\right)}^{b)}+\partial_{\left.d^{\prime}\right)}^{\left.b^{\prime}\right)}\right) A \\
& +\delta_{c d}\left(\partial^{a}+\partial^{a^{\prime}}\right)\left(\partial^{b}+\partial^{b^{\prime}}\right) A-\delta^{a b} \delta_{c d} B .
\end{aligned}
$$

To "trace-reverse" the Green's function according to Eq. (3.24) is a straightforward operation, but it is important to keep in mind that the metric involved in this computation is not $\eta^{\alpha \beta}$, but $\eta^{\alpha \beta}+2 \Phi \chi^{\alpha \beta}$. We find that the gravitational Green's function is given by

$$
G_{\gamma^{\prime} \delta^{\prime}}^{\alpha \beta}\left(x, x^{\prime}\right)=\left(\delta_{\gamma^{\prime}}^{(\alpha} \delta_{\delta^{\prime}}^{\beta)}-\frac{1}{2} \eta^{\alpha \beta} \eta_{\gamma^{\prime} \delta^{\prime}}\right) G^{\mathrm{flat}}\left(x, x^{\prime}\right)+\dot{G}_{\gamma^{\prime} \delta^{\prime}}^{\alpha \beta}\left(x, x^{\prime}\right)+O(\Phi)^{2},
$$

where

$$
\dot{G}_{\gamma^{\prime} \delta^{\prime}}^{\alpha \beta}\left(x, x^{\prime}\right)=\dot{\mathcal{G}}_{\gamma^{\prime} \delta^{\prime}}^{\alpha \beta}\left(x, x^{\prime}\right)-\frac{1}{2} \eta^{\alpha \beta} \eta_{\mu \nu} \dot{\mathcal{G}}_{\gamma^{\prime} \delta^{\prime}}^{\mu \nu}\left(x, x^{\prime}\right)-2\left(t^{\alpha} t^{\beta} \eta_{\gamma^{\prime} \delta^{\prime}}-\eta^{\alpha \beta} t_{\gamma^{\prime}} t_{\delta^{\prime}}\right) \Phi(x) G^{\mathrm{fat}}\left(x, x^{\prime}\right) .
$$

The complete listing of components is

$$
\begin{aligned}
& \dot{G}^{t t}{ }_{t^{\prime} t^{\prime}}=-\left(\Delta \Phi G^{\mathrm{flat}}+\partial_{t t^{\prime}} A\right), \\
& \dot{G}^{t t}{ }_{t^{\prime} a^{\prime}}=\left(\partial_{t^{\prime} a}-\partial_{t a^{\prime}}\right) A, \\
& \dot{G}^{t t}{ }_{a^{\prime} b^{\prime}}=-\delta_{a b}\left[\langle\Phi\rangle G^{\mathrm{flat}}+\partial_{t t^{\prime}} A+2 B\right]+\left(\partial_{a}+\partial_{a^{\prime}}\right)\left(\partial_{b}+\partial_{b^{\prime}}\right) A, \\
& \dot{G}_{t^{\prime} t^{\prime}}^{t a}=\left(\partial^{a}{ }_{t^{\prime}}^{a}-\partial^{a^{\prime}}\right) A, \\
& \dot{G}^{t a}{ }_{t^{\prime} b^{\prime}}=-\delta_{b}^{a}\left(\partial_{t t^{\prime}} A+B\right)+\frac{1}{2}\left(\partial_{b}^{a}+2 \partial_{b}^{a^{\prime}}+\partial_{b^{\prime}}^{a^{\prime}}\right) A, \\
& \dot{G}_{{ }^{\prime}{ }^{\prime} c^{\prime}}=\delta_{(b}^{a}{ }_{(b}\left(\partial_{c) t^{\prime}}-\partial_{\left.c^{\prime}\right) t}\right) A,
\end{aligned}
$$




$$
\begin{aligned}
& \dot{G}_{t^{\prime} t^{\prime}}^{a b}=\delta^{a b}\left[\langle\Phi\rangle G^{\mathrm{flat}}-\partial_{t t^{\prime}} A\right]+\left(\partial^{a}+\partial^{a^{\prime}}\right)\left(\partial_{b}+\partial_{b^{\prime}}\right) A, \\
& \dot{G}_{t^{\prime} c^{\prime}}^{a b}=\delta_{c}^{(a}\left(\partial_{t^{\prime}}^{b)}-\partial_{t}^{\left.b^{\prime}\right)}\right) A \text {, } \\
& \dot{G}^{a b}{ }_{c^{\prime} d^{\prime}}=\left(2 \delta_{c}^{(a} \delta_{d}^{b)}-\delta^{a b} \delta_{c d}\right)\left(\Delta \Phi G^{\mathrm{flat}}-\partial_{t t^{\prime}} A\right)+\delta^{a b}\left(\partial_{c}+\partial_{c^{\prime}}\right)\left(\partial_{d}+\partial_{d^{\prime}}\right) A \\
& -2 \delta_{(c}^{(a}\left(\partial_{d)}^{b)}+2 \partial_{\left.d^{\prime}\right)}^{b)}+\partial_{\left.d^{\prime}\right)}^{\left.b^{\prime}\right)}\right) A+\delta_{c d}\left(\partial^{a}+\partial^{a^{\prime}}\right)\left(\partial^{b}+\partial^{b^{\prime}}\right) A-2 \delta^{a b} \delta_{c d} B .
\end{aligned}
$$

Here we use the notation $\langle\Phi\rangle \equiv \Phi(x)+\Phi\left(x^{\prime}\right)$, as well as $\Delta \Phi=\Phi(x)-\Phi\left(x^{\prime}\right)$. It should be noted that in Eq. (3.32), the part of $\dot{G}_{\gamma^{\prime} \delta^{\prime}}^{\alpha \beta}\left(x, x^{\prime}\right)$ that involves $G^{\text {flat }}\left(x, x^{\prime}\right)$ has support only on the past light cone of the point $x$. The remaining, tail part has support inside the light cone as well. We believe that these results for the gravitational Green's function are new.

\section{EVALUATION OF TWO-POINT FUNCTIONS}

The scalar, electromagnetic, and gravitational Green's functions can all be expressed in terms of $A\left(x, x^{\prime}\right)$ and $B\left(x, x^{\prime}\right)$, the two-point functions introduced in Sec. III A. Our task in this section is to evaluate these two-point functions. We will keep $\Phi(x)$ completely generic, demanding only that far from the matter distribution, it becomes equal to $-M / r$, where $r=|\boldsymbol{x}|$ and $M$ is the total mass. This distinguishes our work from the earlier work of DeWitt and DeWitt [48], who considered only the special case $\Phi=-M / r$. A consequence of keeping $\Phi$ generic is that we will obtain only partial information regarding the two-point functions. This, however, will be sufficient for the computation of the self-forces presented in Sec. V. The results specifically required for this computation are derived in this section. Additional results are presented in Appendix A, which gives an extended discussion of the two-point function $A\left(x, x^{\prime}\right)$. Complete expressions for the two-point functions are derived in Appendix $\mathrm{B}$ for the special case $\Phi=-M / r$; these reproduce the earlier results of DeWitt and DeWitt.

\section{A. Ellipsoidal coordinates}

When Eq. (3.3) is substituted into Eqs. (3.1) and (3.2), we find that the $d t^{\prime \prime}$ integration can be carried out immediately, and we obtain

$$
A\left(x, x^{\prime}\right)=\frac{1}{2 \pi} \int \frac{\Phi\left(\boldsymbol{x}^{\prime \prime}\right)}{\left|\boldsymbol{x}-\boldsymbol{x}^{\prime \prime}\right|\left|\boldsymbol{x}^{\prime \prime}-\boldsymbol{x}^{\prime}\right|} \delta\left(\Delta t-\left|\boldsymbol{x}-\boldsymbol{x}^{\prime \prime}\right|-\left|\boldsymbol{x}^{\prime \prime}-\boldsymbol{x}^{\prime}\right|\right) d^{3} x^{\prime \prime}
$$

and

$$
B\left(x, x^{\prime}\right)=\int \frac{\rho\left(\boldsymbol{x}^{\prime \prime}\right)}{\left|\boldsymbol{x}-\boldsymbol{x}^{\prime \prime}\right|\left|\boldsymbol{x}^{\prime \prime}-\boldsymbol{x}^{\prime}\right|} \delta\left(\Delta t-\left|\boldsymbol{x}-\boldsymbol{x}^{\prime \prime}\right|-\left|\boldsymbol{x}^{\prime \prime}-\boldsymbol{x}^{\prime}\right|\right) d^{3} x^{\prime \prime},
$$

where $\Delta t \equiv t-t^{\prime}$. These are the integrals we will attempt to evaluate. The method of calculation presented below comes from DeWitt and DeWitt [48], but this particular implementation was suggested to us by Alan Wiseman [55].

The $\delta$-function in Eqs. (4.1) and (4.2) enforces the condition $\left|\boldsymbol{x}-\boldsymbol{x}^{\prime \prime}\right|+\left|\boldsymbol{x}^{\prime \prime}-\boldsymbol{x}^{\prime}\right|=\Delta t$, which defines the closed, two-dimensional surface $\mathcal{S}$ formed by the intersection of $x$ 's past light cone with $x^{\prime}$ 's future light cone. This surface is an ellipsoid of revolution centered at $\boldsymbol{x}_{0} \equiv \frac{1}{2}\left(\boldsymbol{x}+\boldsymbol{x}^{\prime}\right)$, of semi-major axis $\frac{1}{2} \Delta t$ and ellipticity $\frac{1}{2}\left|\boldsymbol{x}-\boldsymbol{x}^{\prime}\right|$. To integrate Eqs. (4.1) and (4.2), we will adopt ellipsoidal coordinates adapted to this geometry.

We first summarize our notation:

$$
\Delta t=t-t^{\prime}, \quad \boldsymbol{x}_{0}=\frac{1}{2}\left(\boldsymbol{x}+\boldsymbol{x}^{\prime}\right), \quad \boldsymbol{R}=\boldsymbol{x}-\boldsymbol{x}^{\prime}, \quad R=\left|\boldsymbol{x}-\boldsymbol{x}^{\prime}\right|, \quad \hat{\boldsymbol{n}}=\frac{\boldsymbol{R}}{R}, \quad e=\frac{1}{2} R .
$$

The diagram of Fig. 1 illustrates the situation.

For convenience we orient our coordinate axes such that the unit vector $\hat{\boldsymbol{n}}$ points in the same direction as the $z$, or third, axis; there is no loss of generality involved in this choice. We then represent the vector $\boldsymbol{x}^{\prime \prime}$ in terms of ellipsoidal coordinates $(s, \theta, \phi)$. The coordinate transformation is

$$
\boldsymbol{x}^{\prime \prime}=\boldsymbol{x}_{0}+\boldsymbol{\eta}(s, \theta, \phi),
$$




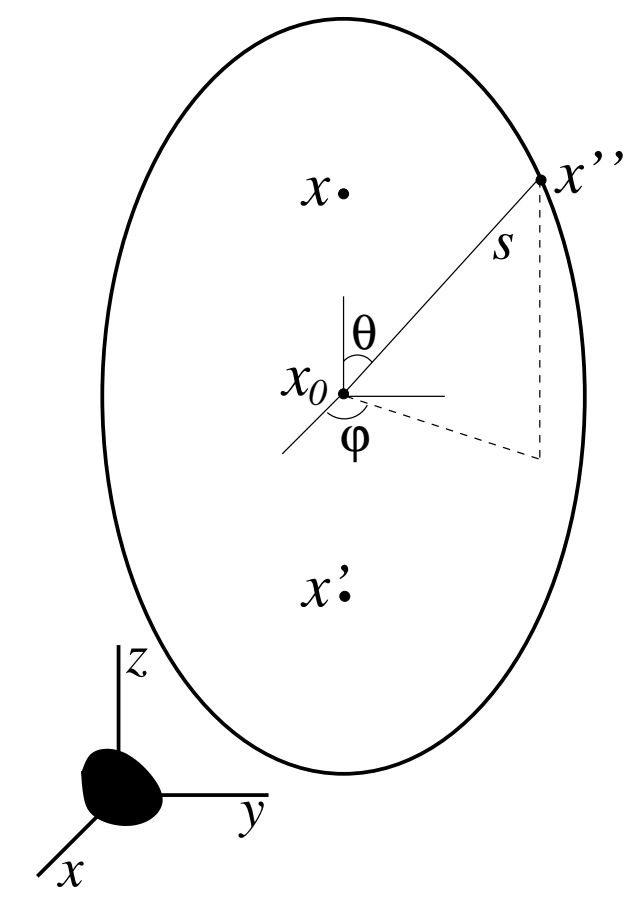

FIG. 1. The diagram shows the matter distribution located near the origin of the Cartesian coordinate system, the points $\boldsymbol{x}, \boldsymbol{x}^{\prime}$, and $\boldsymbol{x}_{0}$, to which the ellipsoidal coordinates $(s, \theta, \phi)$ are attached. The diagram also shows one representative ellipsoid of constant $s$, and a point $\boldsymbol{x}^{\prime \prime}=\boldsymbol{x}_{0}+\boldsymbol{\eta}(s, \theta, \phi)$ lying on this ellipsoid.

where

$$
\eta^{1}=\sqrt{s^{2}-e^{2}} \sin \theta \cos \phi, \quad \eta^{2}=\sqrt{s^{2}-e^{2}} \sin \theta \sin \phi, \quad \eta^{3}=s \cos \theta .
$$

The parameter $e \equiv \frac{1}{2} R$ is the ellipticity of the new coordinate system. It is easy to check that in these coordinates, $\left|\boldsymbol{x}-\boldsymbol{x}^{\prime \prime}\right|=s-e \cos \theta$ and $\left|\boldsymbol{x}^{\prime \prime}-\boldsymbol{x}^{\prime}\right|=s+e \cos \theta$. The Jacobian of the transformation (4.4) is $\left(s^{2}-e^{2} \cos ^{2} \theta\right) \sin \theta$.

With these results, we find that Eq. (4.1) reduces to $A\left(x, x^{\prime}\right)=(2 \pi)^{-1} \int \Phi\left(\boldsymbol{x}_{0}+\boldsymbol{\eta}\right) \delta(\Delta t-2 s) d s d \Omega$, where $d \Omega=$ $\sin \theta d \theta d \phi$ is the element of solid angle. The integration over $s$ is immediate, and we obtain

$$
A\left(x, x^{\prime}\right)=\frac{1}{4 \pi} \int \Phi\left(\boldsymbol{x}_{0}+\boldsymbol{\eta}(\theta, \phi)\right) d \Omega
$$

where the vector $\boldsymbol{\eta}(\theta, \phi)$ is now given by Eq. (4.5) with $s$ set equal to $\frac{1}{2} \Delta t$. Thus, $A\left(x, x^{\prime}\right)$ is the average of $\Phi$ over the ellipsoid $s=\frac{1}{2} \Delta t$, which is just the two-surface $\mathcal{S}$ introduced previously. Similarly,

$$
B\left(x, x^{\prime}\right)=\frac{1}{2} \int \rho\left(\boldsymbol{x}_{0}+\boldsymbol{\eta}(\theta, \phi)\right) d \Omega
$$

is the average of $2 \pi \rho$ over the same ellipsoid. It is important to remember that in Eqs. (4.5)-(4.7), the third coordinate axis has been aligned with the vector $\boldsymbol{R} \equiv \boldsymbol{x}-\boldsymbol{x}^{\prime}$.

It should be noted that $A\left(x, x^{\prime}\right)$ and $B\left(x, x^{\prime}\right)$ are nonzero if and only if $x$ is in the causal future of $x^{\prime}$. To properly reflect this, the integrals of Eqs. (4.6) and (4.7) should be multiplied by the step function $\theta(\Delta t-R)$. To keep the notation simple, however, we choose to leave this factor implicit in our expressions; for the rest of this section, $\Delta t$ will be understood to always be larger than $R$.

\section{B. $A\left(x, x^{\prime}\right)$ near coincidence}

The integral of Eq. (4.6) can readily be evaluated, for an arbitrary Newtonian potential $\Phi$, if the points $x$ and $x^{\prime}$ are close together in spacetime. This is the near-coincidence approximation. Because these points must be timelike related for $A\left(x, x^{\prime}\right)$ to be nonvanishing, we are looking at evaluating Eq. (4.6) in a limit where $R$ and $\Delta t$ are both small, but the ratio $\Delta t / R$ is maintained larger than unity. In this limit, the vector $\boldsymbol{\eta}(\theta, \phi)$ defined in Eq. (4.5) is small, 
and $\Phi$ can be expanded in Taylor series about the point $\boldsymbol{x}_{0}$; this is the basis for our approximation. For concreteness, we will assume that $\boldsymbol{x}_{0}$ lies outside the matter distribution, so that $\rho\left(\boldsymbol{x}_{0}\right)=0$.

Expanding $\Phi$ in powers of $\boldsymbol{\eta}$ in Eq. (4.6) gives

$$
4 \pi A=\Phi\left(\boldsymbol{x}_{\mathbf{0}}\right) \int d \Omega+\Phi_{, a}\left(\boldsymbol{x}_{\mathbf{0}}\right) \int \eta^{a} d \Omega+\frac{1}{2} \Phi_{, a b}\left(\boldsymbol{x}_{\mathbf{0}}\right) \int \eta^{a} \eta^{b} d \Omega+\cdots
$$

The first integral, with a factor of $(4 \pi)^{-1}$ in front, gives unity. The average of $\eta^{a}$ on the sphere is zero, and the average of $\eta^{a} \eta^{b}$ is the tensor $q^{a b}=\frac{1}{3}\left(s^{2}-e^{2}\right) \delta^{a b}+\frac{1}{3} e^{2} \hat{n}^{a} \hat{n}^{b}$, where $s=\frac{1}{2} \Delta t$ and $e=\frac{1}{2} R$. The part of $q^{a b}$ that is proportional to $\delta^{a b}$ multiplies $\Phi_{, a b}\left(\boldsymbol{x}_{0}\right)$ and returns something proportional to $\nabla^{2} \Phi$ evaluated at $\boldsymbol{x}_{0}$; because this point is outside the matter, this is zero. We are left with

$$
A=\Phi\left(\boldsymbol{x}_{0}\right)+\frac{1}{24} R^{2} \Phi_{, a b}\left(\boldsymbol{x}_{\mathbf{0}}\right) \hat{n}^{a} \hat{n}^{b}+\cdots
$$

Notice that the right-hand side of this equation no longer depends on $\Delta t$ : the two-point function $A\left(x, x^{\prime}\right)$ is timeindependent near coincidence.

It is convenient to shift the reference point from $\boldsymbol{x}_{0}$ to $\boldsymbol{x}$. To order $R^{2}$, this means re-expressing $\Phi\left(\boldsymbol{x}_{0}\right)$ as

$$
\Phi\left(\boldsymbol{x}-\frac{1}{2} R \hat{\boldsymbol{n}}\right)=\Phi(\boldsymbol{x})-\frac{1}{2} R \Phi_{, a}(\boldsymbol{x}) \hat{n}^{a}+\frac{1}{8} R^{2} \Phi_{, a b}(\boldsymbol{x}) \hat{n}^{a} \hat{n}^{b}+\cdots .
$$

Substituting this into Eq. (4.9), we arrive at

$$
A\left(x, x^{\prime}\right)=\Phi(\boldsymbol{x})-\frac{1}{2} \Phi_{, a}(\boldsymbol{x}) R^{a}+\frac{1}{6} \Phi_{, a b}(\boldsymbol{x}) R^{a} R^{b}+O\left(R^{3}\right) .
$$

This is our final expression for the two-point function in the near-coincidence approximation. We recall that $\boldsymbol{R}=\boldsymbol{x}-\boldsymbol{x}^{\prime}$.

In the sequel we will need expressions for various derivatives of $A\left(x, x^{\prime}\right)$ evaluated at coincidence, $x=x^{\prime}$. It is straightforward to differentiate Eq. (4.11) with respect to either $x$ or $x^{\prime}$, to obtain

$$
\lim _{x^{\prime} \rightarrow x} \partial_{t} A=\lim _{x^{\prime} \rightarrow x} \partial_{t^{\prime}} A=0
$$

and

$$
\lim _{x^{\prime} \rightarrow x} \partial_{a b} A=2 \lim _{x^{\prime} \rightarrow x} \partial_{a b^{\prime}} A=\lim _{x^{\prime} \rightarrow x} \partial_{a^{\prime} b^{\prime}} A=\frac{1}{3} \Phi_{, a b}(\boldsymbol{x})
$$

These results hold for arbitrary Newtonian potentials, provided that the point $\boldsymbol{x}_{0}$ lies outside the matter distribution.

\section{C. $A\left(x, x^{\prime}\right)$ for long delays}

We can also evaluate $A\left(x, x^{\prime}\right)$ for arbitrary potentials if we assume that $x$ and $x^{\prime}$ are widely separated in spacetime. More precisely, we now take $\Delta t$ to be extremely large, and in particular, much larger than $R=\left|\boldsymbol{x}-\boldsymbol{x}^{\prime}\right|$, $r=|\boldsymbol{x}|$, and $r^{\prime}=\left|\boldsymbol{x}^{\prime}\right|$. This is the long-delay approximation. In this limit, $s$ is much larger than $e$, and $\boldsymbol{\eta} \simeq(s \sin \theta \cos \phi, s \sin \theta \sin \phi, s \cos \theta)$. Because $s$ is very large, we will neglect $\boldsymbol{x}_{0}$ in front of $\boldsymbol{\eta}$ in Eq. (4.6), so that $A \simeq(4 \pi)^{-1} \int \Phi(\boldsymbol{\eta}) d \Omega$. Since $\boldsymbol{\eta}$ is located well outside the matter distribution, we can approximate the Newtonian potential by $-M /|\boldsymbol{\eta}|$, its first term in a multipole expansion. (Here, $M=\int \rho d^{3} x$ is the total mass of the matter distribution.) Because $|\boldsymbol{\eta}| \simeq s=\frac{1}{2} \Delta t$ in the long-delay limit, we arrive at

$$
A\left(x, x^{\prime}\right) \simeq-\frac{2 M}{\Delta t}
$$

We recall that $\Delta t=t-t^{\prime}$. Equation (4.14) shows that $A\left(x, x^{\prime}\right)$ and all its derivatives vanish in the limit $t^{\prime} \rightarrow-\infty$. 


\section{D. $B\left(x, x^{\prime}\right)$ at large distances}

We will evaluate $B\left(x, x^{\prime}\right)$ in the case where $x$ and $x^{\prime}$ are both located well outside the matter distribution. For this calculation it is easier to go back to Eq. (4.2), in which we assume that $r \equiv|\boldsymbol{x}|$ and $r^{\prime} \equiv\left|\boldsymbol{x}^{\prime}\right|$ are both very large. (A more precise criterion will be given below.) These conditions will allow us to develop a multipole expansion for this two-point function.

Equations (4.2) and (4.7) indicate that $B\left(x, x^{\prime}\right)$ is the average of the mass density $\rho$ over the ellipsoid $s=\frac{1}{2} \Delta t$. The diagram of Fig. 1 makes it clear that unless $\Delta t$ falls within the appropriate interval, the ellipsoid will fail to intersect the matter distribution. Thus, $B\left(x, x^{\prime}\right)$ is nonzero only when $\Delta t$ lies within this interval, which we might call the intersection window. When $r$ and $r^{\prime}$ are both large, the ellipsoid is also very large, and the intersection window becomes comparatively short. In this limit, $B\left(x, x^{\prime}\right)$ is "on" for a very short time, and its behavior suggests that of a $\delta$-function. This expectation is borne out by an explicit calculation.

In the denominator of Eq. (4.2), we approximate $\left|\boldsymbol{x}-\boldsymbol{x}^{\prime \prime}\right|$ by $r$ and $\left|\boldsymbol{x}^{\prime \prime}-\boldsymbol{x}^{\prime}\right|$ by $r^{\prime}$, taking $\left|\boldsymbol{x}^{\prime \prime}\right|$ to be much smaller than both $r$ and $r^{\prime}$. In the $\delta$-function we go to the next order of approximation and write $\left|\boldsymbol{x}-\boldsymbol{x}^{\prime \prime}\right| \simeq r-\boldsymbol{x} \cdot \boldsymbol{x}^{\prime \prime} / r$ and $\left|\boldsymbol{x}^{\prime \prime}-\boldsymbol{x}^{\prime}\right| \simeq r^{\prime}-\boldsymbol{x}^{\prime} \cdot \boldsymbol{x}^{\prime \prime} / r^{\prime}$. If we introduce the vector

$$
\boldsymbol{m}=\frac{\boldsymbol{x}}{r}+\frac{\boldsymbol{x}^{\prime}}{r^{\prime}}
$$

then the $\delta$-function becomes $\delta\left(u+\boldsymbol{m} \cdot \boldsymbol{x}^{\prime \prime}\right)$, where $u \equiv \Delta t-r-r^{\prime}$. This we expand in powers of $\boldsymbol{x}^{\prime \prime}$ and express as $\delta(u)+\delta^{\prime}(u)\left(\boldsymbol{m} \cdot \boldsymbol{x}^{\prime \prime}\right)+\frac{1}{2} \delta^{\prime \prime}(u)\left(\boldsymbol{m} \cdot \boldsymbol{x}^{\prime \prime}\right)^{2}+\cdots$, where a prime denotes differentiation with respect to the argument. Substituting all this inside the integral of Eq. (4.2), we obtain

$$
B \simeq \frac{1}{r r^{\prime}}\left[\delta(u) \int \rho\left(\boldsymbol{x}^{\prime \prime}\right) d^{3} x^{\prime \prime}+\delta^{\prime}(u) m_{a} \int \rho\left(\boldsymbol{x}^{\prime \prime}\right) x^{a^{\prime \prime}} d^{3} x^{\prime \prime}+\frac{1}{2} \delta^{\prime \prime}(u) m_{a} m_{b} \int \rho\left(\boldsymbol{x}^{\prime \prime}\right) x^{a^{\prime \prime}} x^{b^{\prime \prime}} d^{3} x^{\prime \prime}+\cdots\right]
$$

The first integral gives the total mass $M$. The second integral gives the dipole moment of the mass distribution; this vanishes if, as we assume, the origin of the coordinate system is attached to the center of mass. The third integral is $Q^{a b}$, the quadrupole moment of the mass distribution. We have therefore obtained

$$
B\left(x, x^{\prime}\right) \simeq \frac{1}{r r^{\prime}}\left[M \delta(u)+\frac{1}{2} Q_{a b} m^{a} m^{b} \delta^{\prime \prime}(u)+\cdots\right],
$$

where, we recall, $u=\Delta t-r-r^{\prime}$. This equation displays the first two terms in the multipole expansion of the two-point function $B\left(x, x^{\prime}\right)$. Notice that this expansion is analogous to a multipole expansion of a radiative field in the wave zone; this is not a near-zone expansion of a quasi-stationary field.

In the sequel we will approximate $B\left(x, x^{\prime}\right)$ by

$$
B\left(x, x^{\prime}\right)=\frac{M}{r r^{\prime}} \delta\left(\Delta t-r-r^{\prime}\right)
$$

its leading term in the multipole expansion. In this approximation, $B\left(x, x^{\prime}\right)$ is nonzero only when $\Delta t=r+r^{\prime}$. The time delay corresponds to a signal propagating at the speed of light from $\boldsymbol{x}^{\prime}$ to the center of the mass distribution, and then on to $\boldsymbol{x}$. The approximation has made the intersection window infinitely short.

To estimate the error involved in going from Eq. (4.17) to Eq. (4.18), we integrate $B\left(x, x^{\prime}\right)$ over a smooth test function $f(t)$. Setting $t^{\prime}=0$ for simplicity, Eq. (4.17) gives

$$
\int B f(t) d t \simeq \frac{M}{r r^{\prime}} f\left(r+r^{\prime}\right)\left(1+\frac{Q}{M} \frac{f^{\prime \prime}}{f}+\cdots\right)
$$

where $Q \equiv \frac{1}{2} Q_{a b} m^{a} m^{b}$. The factor in front the large brackets is what would be returned by the approximation of Eq. (4.18). We wish to show that in typical situations, the second term inside the brackets is much smaller than unity. Let $\ell$ be the characteristic size of the matter distribution, and let $\tau$ be the characteristic time over which the function $f(t)$ changes. Then $Q / M$ is of order $\ell^{2}$, and $f^{\prime \prime} / f$ is of order $1 / \tau^{2}$. The correction term is therefore of order $(\ell / \tau)^{2}$. In a typical situation, $\tau$ would be associated with the orbital time scale of an object located at $\boldsymbol{x}$ or $\boldsymbol{x}^{\prime}$. Assuming for concreteness that $r$ is the shortest distance, we have $\tau^{2} \sim r^{3} / M$. With this, we find that the correction term in Eq. (4.19) is of the order of $(\ell / r)^{2}(M / r)$. So long as $r$ and $r^{\prime}$ are both much larger than $\ell$ and $M$, Eq. (4.18) makes an excellent approximation. 


\section{SELF-FORCES}

\section{A. Scalar self-force}

In the absence of an external force, a point particle with scalar charge $q$ experiences a self-force 46.

$$
f^{\alpha}(\tau)=q^{2}\left[g^{\alpha \beta}+u^{\alpha}(\tau) u^{\beta}(\tau)\right] \int_{-\infty}^{\tau^{-}} G_{, \beta}\left(\tau, \tau^{\prime}\right) d \tau^{\prime},
$$

so that its equations of motion are $m u_{; \beta}^{\alpha} u^{\beta}=f^{\alpha}$. The particle's world line is represented by the relations $z^{\alpha}(\tau)$, where $\tau$ is proper time, and $u^{\alpha}(\tau)=d z^{\alpha} / d \tau$. The self-force is evaluated at the current position of the particle, and it involves an integration over its past history. Inside the integral, the retarded Green's function is first written as $G\left(x, z\left(\tau^{\prime}\right)\right)$, in terms of an arbitrary field point $x$ and the past position $z\left(\tau^{\prime}\right)$ of the particle. The Green's function is then differentiated with respect to $x^{\beta}$, and the result is evaluated at $x=z(\tau)$, the particle's current position. This is what we have denoted by $G_{, \beta}\left(\tau, \tau^{\prime}\right)$ in Eq. (5.1). The upper limit of integration is $\tau^{-} \equiv \tau-\epsilon$, where $\epsilon$ is a small, positive number that is taken to zero at the end of the calculation. The purpose of this cutoff is to remove the singular, $\delta$-function part of the Green's function which has support on the past light cone of the point $z(\tau)$. What survives is the "tail part" of the Green's function, which has support inside the light cone.

This expression for the self-force was derived by Quinn [46], who assumed that the scalar field was minimally coupled to curvature - he considered the special case $\xi=0$. There is no difficulty, however, in extending Quinn's result to arbitrary couplings to curvature. So long as the particle moves in a region of spacetime in which the Ricci scalar is zero, his results carry over directly. The only effect that $\xi$ has on the motion is through the Green's function, which is sensitive to a nonzero Ricci scalar somewhere in spacetime.

We will calculate $f^{\alpha}$ to first order in the Newtonian potential $\Phi$ in a weak-field approximation; the particle therefore moves in the weakly curved spacetime of Sec. II. We assume that the particle is gravitationally bound to the matter distribution, and infer from the virial theorem that $v^{2}$, the square of the particle's velocity, is of the same order of magnitude as $\Phi$. We therefore add a slow-motion assumption to our weak-field assumption. Accordingly, we will neglect in all expressions terms involving $\Phi^{2}, \Phi v^{2}$, and $v^{4}$. Within this approximation, we have that the spatial components of the self-force are given by

$$
f^{a}=q^{2} \int_{-\infty}^{t}\left(\dot{G}_{, a}+v^{a} \dot{G}_{, t}\right) d t^{\prime},
$$

where $\boldsymbol{v}=d \boldsymbol{z}(t) / d t$ is the current velocity of the particle, which does not depend on $t^{\prime}$; the past velocity will be denoted $\boldsymbol{v}^{\prime}=d \boldsymbol{z}\left(t^{\prime}\right) / d t^{\prime}$, and this does depend on $t^{\prime}$. We have made $t^{\prime}$ the new variable of integration, and neglected all $O\left(v^{2}, v v^{\prime}, v^{\prime 2}\right)$ terms inside the integral, which through the Green's function is already linear in the Newtonian potential. This fact allows us to freely alter the position of all latin indices, implicitly using $\delta_{a b}$, the metric of flat space. And since we will substitute only the tail part of the Green's function inside the integral, we have made $t$ the upper limit of integration (instead of the redundant $t-\epsilon$ ).

From Eq. (3.14) we find that the tail part of the scalar Green's function is given by

$$
\dot{G}\left(x, z^{\prime}\right)=-2 \partial_{t t^{\prime}} A\left(x, z^{\prime}\right)-2 \xi B\left(x, z^{\prime}\right) .
$$

Here $z^{\prime} \equiv\left(t^{\prime}, \boldsymbol{z}\left(t^{\prime}\right)\right)$, and $A\left(x, z^{\prime}\right), B\left(x, z^{\prime}\right)$ are the two-point functions introduced in Sec. III A and computed in Sec. IV; we will set $x=z \equiv(t, \boldsymbol{z}(t))$ after taking the derivatives. Substituting Eq. (5.3) into Eq. (5.2) gives $f^{a}=f_{A}^{a}+f_{B}^{a}$, where

$$
f_{A}^{a}=-2 q^{2} \int_{-\infty}^{t}\left(A_{, t t^{\prime} a}+v^{a} A_{, t t^{\prime} t}\right) d t^{\prime}
$$

and

$$
f_{B}^{a}=-2 \xi q^{2} \int_{-\infty}^{t}\left(B_{, a}+v^{a} B_{, t}\right) d t^{\prime}
$$

We will first work on $f_{A}^{a}$, and then move on to $f_{B}^{a}$.

The first term inside the integral of Eq. (5.4) can be expressed as $\partial_{t^{\prime}} A_{, t a}=d A_{, t a} / d t^{\prime}-A_{, t a b^{\prime}} v^{b^{\prime}}$, allowing part of the integration to be carried out. Similarly, we write the second term inside the integral as $v^{a} \partial_{t^{\prime}} A_{, t t}=v^{a} d A_{, t t} / d t^{\prime}+O\left(v v^{\prime}\right)$, and Eq. (5.4) becomes 


$$
f_{A}^{a}=-2 q^{2}\left[A_{, t a}+v^{a} A_{, t t}\right]_{-\infty}^{t}+2 q^{2} \int_{-\infty}^{t} A_{, t a b^{\prime}} v^{b^{\prime}} d t^{\prime} .
$$

We now use Eq. (3.6) and replace $A_{, t a b^{\prime}}$ by $-\partial_{t^{\prime}} A_{, a b^{\prime}}=-d A_{, a b^{\prime}} / d t^{\prime}+O\left(v^{\prime}\right)$. After integrating by parts, we obtain

$$
f_{A}^{a}=-2 q^{2}\left[A_{, t a}+v^{a} A_{, t t}+A_{, a b^{\prime}} v^{b^{\prime}}\right]_{-\infty}^{t}+2 q^{2} \int_{-\infty}^{t} A_{, a b^{\prime}} a^{b^{\prime}} d t^{\prime},
$$

where $\boldsymbol{a}^{\prime}=d \boldsymbol{v}^{\prime} / d t^{\prime}$ is the past acceleration of the particle. Because the particle is not subjected to any force except for gravity, its acceleration is linear in the Newtonian potential $\Phi$. Since $A\left(x, z^{\prime}\right)$ is already linear in $\Phi$, the integral of Eq. (5.7) is $O\left(\Phi^{2}\right)$ and it can be neglected. The first part of the self-force can therefore be computed without having to evaluate a single integral! And Eq. (5.7) shows that the self-force depends on the conditions at the current time, and in the infinite past. At this stage, the nonlocal character of the force has (almost) disappeared.

In Sec. IV $\mathrm{C}$ we learned that as $t^{\prime}$ approaches $-\infty, A\left(x, z^{\prime}\right)$ behaves as $2 M / t^{\prime}$. This shows that in Eq. (5.7), there are actually no contributions from the infinite past. Any nonvanishing contribution must therefore come from the current time, $t^{\prime}=t$, at which the points $\boldsymbol{z}\left(t^{\prime}\right)$ and $\boldsymbol{z}(t)$ are coincident. Equation (4.12) tells us that the time derivative of $A\left(x, z^{\prime}\right)$ is zero at coincidence. Setting $v^{b^{\prime}}=v^{b}$, we find that the sole surviving contribution to $f_{A}^{a}$ is $-2 q^{2} A_{, a b^{\prime}} v^{b}$, in which the derivatives of the two-point function must be evaluated at coincidence. This was worked out in Eq. (4.13), and we arrive at

$$
f_{A}^{a}=-\frac{1}{3} q^{2} \Phi_{, a b} v^{b}
$$

We may express this in the alternative form

$$
\boldsymbol{f}_{A}=\frac{1}{3} q^{2} \frac{d \boldsymbol{g}}{d t}
$$

where $\boldsymbol{g}=-\boldsymbol{\nabla} \Phi$ is the Newtonian gravitational field. In components, the right-hand side of Eq. (5.9) involves $d g^{a} / d t=-d \Phi_{, a} / d t=-\Phi_{, a b} v^{b}$, where we have used the fact that the Newtonian potential does not explicitly depend on time.

We now turn to the second part of the self-force, given by Eq. (5.5) in terms of the two-point function $B\left(x, z^{\prime}\right)$. According to Eq. (4.18), this is

$$
B\left(x, z^{\prime}\right)=\frac{M}{r r^{\prime}} \delta(u),
$$

where $u=t-t^{\prime}-r-r^{\prime}, r=|\boldsymbol{x}|$, and $r^{\prime}=\left|\boldsymbol{z}\left(t^{\prime}\right)\right|$. As before, the field point $x=(t, \boldsymbol{x})$ will be identified with $z \equiv(t, \boldsymbol{z}(t))$ after differentiation. It is important to keep in mind that in Eq. (5.10), $r^{\prime}$ depends on $t^{\prime}$. We will use the notation $v_{r}^{\prime}=d r^{\prime} / d t^{\prime}$.

We first show that in Eq. (5.5), the integral involving the time derivative of $B\left(x, z^{\prime}\right)$ is negligible. According to Eq. (5.10), $B, t=M \delta^{\prime}(u) /\left(r r^{\prime}\right)$, where a prime indicates differentiation with respect to $u$. Changing the variable of integration from $t^{\prime}$ to $u$, using $d u=-\left(1+v_{r}^{\prime}\right) d t^{\prime}$, we have that $\int B_{, t} d t^{\prime}=(M / r) \int\left(r^{\prime}\right)^{-1}\left[1+O\left(v^{\prime}\right)\right] \delta^{\prime}(u) d u$. Here the lower limit of integration is $-\left(r+r^{\prime}\right)$, the upper limit is $\infty$, and we neglect terms of order $v^{\prime}$ because the integral comes with a factor of $v^{a}$ in front. Integration yields $M v_{r}^{\prime} /\left(r r^{\prime 2}\right)$ evaluated at $u=0$. We conclude that the second term of Eq. (5.5) is of order $(q / r)^{2}(M / r) v^{2}$ and therefore negligible.

Differentiation of Eq. (5.10) with respect to the spatial variables $x^{a}$ yields

$$
B_{, a}=-\frac{M r_{, a}}{r^{2} r^{\prime}}\left[\delta(u)+r \delta^{\prime}(u)\right],
$$

where a prime still indicates differentiation with respect to $u$. Changing variables of integration from $t^{\prime}$ to $u$, we find that the first integral of Eq. (5.5) becomes

$$
\begin{aligned}
\int_{-\infty}^{t} B_{, a} d t^{\prime} & =-\frac{M r_{, a}}{r^{2}} \int_{-\left(r+r^{\prime}\right)}^{\infty} \frac{\delta(u)+r \delta^{\prime}(u)}{r^{\prime}\left(1+v_{r}^{\prime}\right)} d u \\
& =-\left.\frac{M r_{, a}}{r^{2}}\left[\frac{1}{r^{\prime}\left(1+v_{r}^{\prime}\right)}-r \frac{d}{d u} \frac{1}{r^{\prime}\left(1+v_{r}^{\prime}\right)}\right]\right|_{u=0} \\
& =-\left.\frac{M r_{, a}}{r^{2} r^{\prime}}\left[1-\left(1+r / r^{\prime}\right) v_{r}^{\prime}+O\left(v^{\prime 2}, a^{\prime}\right)\right]\right|_{u=0}
\end{aligned}
$$


where, as indicated, we neglect terms that are of the second order in the velocity, and terms that involve the particle's acceleration.

To evaluate the right-hand side of Eq. (5.12) is made difficult by the fact that the relation $u=t-t^{\prime}-r-r^{\prime}\left(t^{\prime}\right)$ cannot be inverted for $t^{\prime}$, because the function $r^{\prime}\left(t^{\prime}\right)$ is not known. For the purposes of this calculation, however, it is sufficient to give an approximate inversion. We let the radial part of the particle's trajectory be described by the relation $r=R(t)$, so that $r^{\prime}=R\left(t^{\prime}\right)=R(t-\Delta t)$. This we approximate by $R(t)-\dot{R}(t) \Delta t+\frac{1}{2} \ddot{R}(t) \Delta t^{2}+\cdots$, where overdots indicate differentiation with respect to $t$. Neglecting the acceleration term, we have $r^{\prime}=r-v_{r} \Delta t+O(a)$, where $v_{r} \equiv \dot{R}(t)$. Similarly, we obtain $v_{r}^{\prime} \equiv \dot{R}\left(t^{\prime}\right)=v_{r}+O(a)$. When $u=0$, we find that $\Delta t=2 r /\left(1+v_{r}\right)+O(a)=$ $2 r\left(1-v_{r}\right)+O\left(v^{2}, a\right)$, giving $r^{\prime}=r\left(1-2 v_{r}\right)+O\left(v^{2}, a\right)$. Substituting this into Eq. (5.12), we find

$$
\int_{-\infty}^{t} B_{, a} d t^{\prime}=-\frac{M r_{, a}}{r^{3}}\left[1+O\left(v^{2}, a\right)\right]
$$

Finally, going back to Eq. (5.5), we arrive at

$$
\boldsymbol{f}_{B}=2 \xi q^{2} \frac{M}{r^{3}} \hat{\boldsymbol{r}}
$$

where $\hat{\boldsymbol{r}}=\boldsymbol{x} / r$ is a unit vector pointing in the radial direction; its components are equal to $x^{a} / r=r_{, a}$. is

The total self-force acting on a point particle with scalar charge $q$ is given by the sum of Eqs. (5.9) and (5.14). This

$$
\boldsymbol{f}=2 \xi q^{2} \frac{M}{r^{3}} \hat{\boldsymbol{r}}+\frac{1}{3} q^{2} \frac{d \boldsymbol{g}}{d t}
$$

where, we recall, $\boldsymbol{g}=-\boldsymbol{\nabla} \Phi$ is the Newtonian gravitational field. The equations of motion for the particle are then $d^{2} \boldsymbol{z} / d t^{2}=\boldsymbol{g}+\boldsymbol{f} / m$, where $m$ is its mass. The first term on the right-hand side of Eq. (5.15) is a conservative correction to the local gravity. This is a repulsive force if $\xi>0$, an attractive force if $\xi<0$, and this part of the self-force disappears altogether if the scalar field is minimally coupled to curvature. The second term is nonconservative; this is the well-known expression for the radiation-reaction force experienced by a scalar charge moving under the influence of an external force $m \boldsymbol{g}$.

\section{B. Electromagnetic self-force}

The steps required to calculate the electromagnetic self-force are very similar to those presented in the preceding subsection. Our discussion here will therefore be brief. We employ the same notation, and work within the same set of approximations.

Our starting point is the DeWitt-Brehme expression for the self-force experienced by a point particle with an electric charge $e$ [4]:

$$
f^{\alpha}=-e^{2} \int_{-\infty}^{\tau^{-}}\left(G_{\gamma^{\prime} ; \beta}^{\alpha}-G_{\beta \gamma^{\prime}}^{; \alpha}\right) u^{\beta} u^{\gamma^{\prime}} d \tau^{\prime}
$$

where $G_{\beta^{\prime}}^{\alpha}\left(\tau, \tau^{\prime}\right)$ is the retarded Green's function of the electromagnetic field, $u^{\alpha}=d z^{\alpha}(\tau) / d \tau$ is the current fourvelocity of the charged particle, and $u^{\alpha^{\prime}}=d z^{\alpha}\left(\tau^{\prime}\right) / d \tau^{\prime}$ its past four-velocity. With the approximations introduced in Sec. V A, we find that the spatial components of the self-force are given by

$$
f^{a}=-e^{2} \int_{-\infty}^{t}\left[\left(\dot{G}_{a t^{\prime}, t}-\dot{G}_{t t^{\prime}, a}\right)+\left(\dot{G}_{a t^{\prime}, b}-\dot{G}_{b t^{\prime}, a}\right) v^{b}+\left(\dot{G}_{a b^{\prime}, t}-\dot{G}_{t b^{\prime}, a}\right) v^{b^{\prime}}\right] d t^{\prime}
$$

only the tail part of the Green's function appears under the integral. Using Eq. (3.21), we find that the self-force can again be written as a sum of two parts, $\boldsymbol{f}=\boldsymbol{f}_{A}+\boldsymbol{f}_{B}$, where

$$
f_{A}^{a}=e^{2} \int_{-\infty}^{t}\left[\left(A_{, t t^{\prime} a}+A_{, t t a^{\prime}}\right)+\left(A_{, t a^{\prime} b}-A_{, t a b^{\prime}}\right) v^{b}+\left(2 \delta_{a b} A_{, t t^{\prime} t}-A_{, t a^{\prime} b}+2 A_{, t a b^{\prime}}-A_{, t^{\prime} a b}\right) v^{b^{\prime}}\right] d t^{\prime}
$$

and 


$$
f_{B}^{a}=-e^{2} \int_{-\infty}^{t}\left(B_{, a}-B_{, t} v^{a^{\prime}}\right) d t^{\prime}
$$

The evaluation of $\boldsymbol{f}_{B}$ proceeds as in Sec. V A, using Eq. (5.13) and the fact that the second term does not contribute to the force at this level of approximation. The result is $f_{B}^{a}=e^{2}\left(M / r^{3}\right) r_{, a}$. The evaluation of $\boldsymbol{f}_{A}$ also involves familiar steps, which we now describe.

The first group of terms inside the integral of Eq. (5.18) are expressed as

$$
\partial_{t^{\prime}}\left(A_{, t a}-A_{, t a^{\prime}}\right)=\frac{d}{d t^{\prime}}\left(A_{, t a}-A_{, t a^{\prime}}\right)-\left(A_{, t a b^{\prime}}-A_{, t a^{\prime} b^{\prime}}\right) v^{b^{\prime}} .
$$

The first term is immediately integrated, and by virtue of Eq. (4.12), this gives no contribution to the self-force. The remaining terms are absorbed into the third group of terms in Eq. (5.18), those which are proportional to $v^{b^{\prime}}$. In the second group of terms, the quantity in front of $v^{b}$ is expressed as

$$
-\partial_{t^{\prime}}\left(A_{, a^{\prime} b}-A_{, a b^{\prime}}\right)=-\frac{d}{d t^{\prime}}\left(A_{, a^{\prime} b}-A_{, a b^{\prime}}\right)+O\left(v^{\prime}\right) .
$$

This is also immediately integrated. Equation (4.13) shows that this also gives no contribution to the self-force. We are therefore left with the group of terms proportional to $v^{b^{\prime}}$. This takes the form of $\left(d C_{a b} / d t^{\prime}\right) v^{b^{\prime}}$, where

$$
C_{a b}=2 \delta_{a b} A_{, t t}-A_{, a b}-A_{, a b^{\prime}}+A_{, a^{\prime} b}-A_{, a^{\prime} b^{\prime}} .
$$

The time derivative is then transferred to $v^{b^{\prime}}$ by integration by parts. This produces an integral that can be neglected, and boundary terms at $t^{\prime}=t$ (the boundary terms at $t^{\prime}=-\infty$ all vanish). Those give $C_{a b} v^{b}$ evaluated at coincidence. Using Eqs. (4.12) and (4.13) we find that Eq. (5.18) becomes $f_{A}^{a}=-\frac{2}{3} e^{2} \Phi_{, a b} v^{b}$.

We have found that the self-force acting on a point particle with electric charge $e$ is given by

$$
\boldsymbol{f}=e^{2} \frac{M}{r^{3}} \hat{\boldsymbol{r}}+\frac{2}{3} e^{2} \frac{d \boldsymbol{g}}{d t},
$$

where $\hat{\boldsymbol{r}}=\boldsymbol{x} / r$ and $\boldsymbol{g}=-\boldsymbol{\nabla} \Phi$ is the Newtonian gravitational field. The first term on the right-hand side of Eq. (5.23) represents a repulsive, conservative force. The second term gives the well-known expression for the radiation-reaction force experienced by an electric charge moving under the influence of an external force $m \boldsymbol{g}$ [43, 44 .

Equation (5.23) implies that the force required to keep an electric charge static in the gravitational field of an isolated object of mass $M$ must be smaller than $m g$ by $e^{2} M / r^{3}$. Our derivation indicates that this correction is caused by an interaction between the electromagnetic field and the matter distribution; this interpretation is suggested by the fact that this contribution to the self-force can be traced back to the two-point function $B\left(x, x^{\prime}\right)$, which is directly associated with $\rho$, the mass density. While this is a valid interpretation, it is interesting to note that the correction persists even in the total absence of matter, although this cannot be revealed in a weak-field approach. The force required to hold an electric charge in place in the exact field of a Schwarzschild black hole was calculated by Smith and Will [49]. In the weak-field limit, these authors recover the conservative term in Eq. (5.23). In this case, clearly, the effect has nothing to do with the presence of matter. It must instead be attributed to an interaction between the electromagnetic field and the hole's event horizon. That the conservative self-force should be so insensitive to the source of the gravitational field is quite interesting. This robustness, however, doesn't seem to apply to scalar charges: The extra force required to keep a scalar charge in place in a Schwarzschild spacetime is always zero, irrespective of the coupling to curvature $[47,55$ 58]. This is quite different from what was obtained in Sec. V A, where we found that in the presence of matter, there is a nonzero self-force for nonminimal coupling.

\section{Gravitational self-force}

The self-force experienced by a point particle of mass $m$ moving in a curved spacetime is given by [26,27]

$$
f^{\alpha}=-2 m^{2} \int_{-\infty}^{\tau^{-}}\left(2 G_{\beta \mu^{\prime} \nu^{\prime} ; \gamma}^{\alpha}-G_{\beta \gamma \mu^{\prime} \nu^{\prime}}{ }^{\alpha}+u^{\alpha} G_{\beta \gamma \mu^{\prime} \nu^{\prime} ; \delta} u^{\delta}\right) u^{\beta} u^{\gamma} u^{\mu^{\prime}} u^{\nu^{\prime}} d \tau^{\prime}
$$

where $G_{\mu^{\prime} \nu^{\prime}}^{\alpha \beta}\left(\tau, \tau^{\prime}\right)$ is the gravitational Green's function, $u^{\alpha}$ the current four-velocity of the particle, and $u^{\mu^{\prime}}$ its past four-velocity. This result was derived for the first time by Mino, Sasaki, and Tanaka [26], although an incomplete 
attempt was made earlier by Morette-DeWitt and Ging [59]. The gravitational self-force was later recovered by Quinn and Wald [27], who gave a more transparent derivation. Their expression is technically wrong, however, because it incorrectly puts $\mathcal{G}_{\mu^{\prime} \nu^{\prime}}^{\alpha \beta}$, the trace-reversed Green's function (see Sec. III D), in place of $G^{\alpha \beta}{ }_{\mu^{\prime} \nu^{\prime}}$ in Eq. (5.24); this slight oversight hardly diminishes the value of their paper.

Our expression for the gravitational self-force differs from Mino et al. [26] in two ways. First, we use a different normalization for the Green's function, and Eq. (5.24) is valid in the normalization imposed by Eq. (3.25). Second, and more importantly, both Mino et al. and Quinn \& Wald [27] assume that the background metric $g_{\alpha \beta}$ is a solution to the Einstein field equations in vacuum, a condition that is incompatible with the weak-field approximation adopted in this paper. [Please refer back to the discussion presented in the paragraphs before Eq. (1.13).] As we have already indicated, the extension of the Mino-Sasaki-Tanaka-Quinn-Wald equations of motion to spacetimes that contain matter is not entirely trivial. In this subsection we consider only the straightforward modifications to the Green's function that come from the presence of Ricci-tensor terms in Eq. (2.13). In other words, we still define the gravitational self-force by Eq. (5.24), but we substitute in it the Green's function computed in Sec. III D to account for the presence of matter. In Sec. VI we shall prove that this substitution is appropriate, and derive additional (matter-mediated) corrections to the equations of motion.

Under the weak-field, slow-motion approximation, we find that the spatial components of the self-force are given by

$$
\begin{aligned}
f^{a}= & -2 m^{2} \int_{-\infty}^{t}\left[\left(2 \dot{G}_{a t t^{\prime} t^{\prime}, t}-\dot{G}_{t t t^{\prime} t^{\prime}, a}\right)+\left(\delta_{a b} \dot{G}_{t t t^{\prime} t^{\prime}, t}+2 \dot{G}_{a b t^{\prime} t^{\prime}, t}+2 \dot{G}_{a t t^{\prime} t^{\prime}, b}-2 \dot{G}_{b t t^{\prime} t^{\prime}, a}\right) v^{b}\right. \\
& \left.+2\left(2 \dot{G}_{a t b^{\prime} t^{\prime}, t}-\dot{G}_{t t b^{\prime} t^{\prime}, a}\right) v^{b^{\prime}}\right] d t^{\prime} .
\end{aligned}
$$

With the tail part of the Green's function extracted from Eq. (3.32), we have $\boldsymbol{f}=\boldsymbol{f}_{A}+\boldsymbol{f}_{B}$, where

$$
\begin{aligned}
f_{A}^{a}= & -2 m^{2} \int_{-\infty}^{t}\left[\left(2 A_{, t t a^{\prime}}-A_{, t t^{\prime} a}\right)+\left(-3 \delta_{a b} A_{, t t^{\prime} t}+2 A_{, t a b}+4 A_{, t a^{\prime} b}+2 A_{, t a^{\prime} b^{\prime}}\right) v^{b}\right. \\
& \left.+2\left(2 \delta_{a b} A_{, t t^{\prime} t}-2 A_{, t a^{\prime} b}-A_{, t a^{\prime} b^{\prime}}+A_{, t a b^{\prime}}\right) v^{b^{\prime}}\right] d t^{\prime}
\end{aligned}
$$

and

$$
f_{B}^{a}=-8 m^{2} \int_{-\infty}^{t} B_{, t} v^{a^{\prime}} d t^{\prime}
$$

Referring back to the discussion preceding Eq. (5.11), we see that $\boldsymbol{f}_{B}$ is of the order of $\left(m M / r^{2}\right)(m / r) v^{2}$, which is a correction of order $\Phi v^{2}$ relative to Newtonian gravity. This gives a second post-Newtonian (2PN) correction [52] to the Newtonian equations of motion, and according to our approximation rules, this must be neglected.

The evaluation of $\boldsymbol{f}_{A}$ is lengthier, but the steps are familiar. The first group of terms inside the integral of Eq. (5.26) are

$$
-\partial_{t^{\prime}}\left(2 A_{, t a^{\prime}}+A_{, t a}\right)=-\frac{d}{d t^{\prime}}\left(2 A_{, t a^{\prime}}+A_{, t a}\right)+\left(2 A_{, t a^{\prime} b^{\prime}}+A_{, t a b^{\prime}}\right) v^{b^{\prime}} .
$$

The total derivatives contributes nothing to the self-force, and the remaining terms are absorbed into the third group. In the second group, the quantity in front of $v^{b}$ is

$$
-\frac{d}{d t^{\prime}}\left(-3 \delta_{a b} A_{, t t^{\prime}}+2 A_{, a b}+4 A_{, a^{\prime} b}+2 A_{, a^{\prime} b^{\prime}}\right)+O\left(v^{\prime}\right),
$$

and this group contributes $4 m^{2} \Phi_{, a b} v^{b}$ to the self-force. Finally, the third group of terms is

$$
2 v^{b^{\prime}} \frac{d}{d t^{\prime}}\left(2 \delta_{a b} A_{, t t}+2 A_{, a^{\prime} b}-\frac{3}{2} A_{, a b^{\prime}}\right)+O\left(v^{\prime 2}\right),
$$

and after integrating by parts, we find that this contributes $-\frac{1}{3} m^{2} \Phi_{, a b} v^{b}$ to the self-force. Summing the contributions gives $f_{A}^{a}=\frac{11}{3} m^{2} \Phi_{, a b} v^{b}$.

The gravitational self-force acting on a point particle of mass $m$ is therefore

$$
\boldsymbol{f}=-\frac{11}{3} m^{2} \frac{d \boldsymbol{g}}{d t},
$$


where $\boldsymbol{g}=-\nabla \Phi$. This gives a correction of $1.5 \mathrm{PN}$ order to the equations of motion, and appears to represent radiation reaction. According to the naive equations of motion $d^{2} \boldsymbol{z} / d t^{2}=\boldsymbol{g}+\boldsymbol{f} / m$, this term does work on the particle at an average rate $d W / d t=+\frac{11}{3} m^{2}|\boldsymbol{g}|^{2}$. We see that the self-force of Eq. (5.31) seems to bring energy to the particle.

There are two major problems of interpretation associated with Eq. (5.31). First, the self-force seems to give rise to radiation antidamping instead of radiation damping [51]. Second, radiation reaction seems to occur at 1.5PN order, while it is known that the effect should appear only at 2.5PN order in a post-Newtonian expansion of the relativistic equations of motion [3 5, 11]. In the next section we shall see that these problems disappear once we properly incorporate additional (matter-mediated) corrections to the equations of motion.

\section{MASSIVE PARTICLE IN A SPACETIME CONTAINING MATTER}

Our task in this section is to produce a proper extension of the Mino-Sasaki-Tanaka-Quinn-Wald equations of motion [26,27 to spacetimes that contain matter. First (Secs. VI A and B), we establish that in this context, the gravitational self-force $f_{\text {self }}^{\alpha}$ is still given by Eq. (1.3), but that the retarded Green's function must be a solution to Eq. (3.22), which includes Ricci-tensor terms generated by the matter. That this does indeed give the correct expression for the self-force was presented as an assumption in the preceding sections of this paper. Second (Sec. VI C), we derive the existence of an additional term in the equations of motion, which now take the form

$$
m u_{; \beta}^{\alpha} u^{\beta}=f_{\text {self }}^{\alpha}+f_{\mathrm{mm}}^{\alpha},
$$

where $f_{\mathrm{mm}}^{\alpha}$ is the matter-mediated force alluded to previously. This force originates from the change in the background metric associated with the motion of the mass $M$ around the system's center of mass; this motion is caused by the gravitational action of the particle on the central mass. Third (Secs. VI D to H), we compute the matter-mediated force, and show that it contains a radiation-damping term that precisely cancels the antidamping term in the self-force. Such a cancellation was noticed a long time ago by Carmeli [53], but in the context of a very different formulation of the equations of motion. We will see (Sec. VI I) that within the weak-field, slow-motion approximation considered here, Eq. 6.1) reduces to the appropriate limit of the standard post-Newtonian equations of motion [3]. 5 . 11.

\section{A. Background spacetime and test particle}

Let us consider the background spacetime first. We are given a metric $g_{\alpha \beta}$ that satisfies the Einstein field equations in the presence of matter. We assume that the matter distribution is bounded and describes an isolated object of mass $M$; throughout this section we will refer to this object as "the star". We write the field equations as $G^{\alpha \beta}[g]=8 \pi T^{\alpha \beta}[g]$, and indicate explicitly that the stress-energy tensor is a functional of the metric. For the moment we take $g_{\alpha \beta}$ to be an exact solution to the field equations; the weak-field approximation will be incorporated at a later stage. And for the moment we avoid adopting a specific phenomenology for the stress-energy tensor; the star can always be thought of as a ball of perfect fluid with a specified equation of state.

In this spacetime we insert a point particle of mass $m$. We assume that this particle moves on a world line $z^{\alpha}(\tau)$ in a region of spacetime that is empty of matter; the parameter $\tau$ is the particle's proper time. We write the particle's stress-energy tensor as

$$
\tau^{\alpha \beta}(x)=m \int u^{\alpha} u^{\beta}(-g)^{-1 / 2} \delta_{4}(x-z(\tau)) d \tau,
$$

where $u^{\alpha}(\tau)=d z^{\alpha} / d \tau$ is the four-velocity and the $\delta$-function is a four-dimensional distribution with support on the world line. It is easy to show that conservation of the stress-energy tensor in the background spacetime implies geodesic motion:

$$
\tau_{; \beta}^{\alpha \beta}=m \int u_{; \beta}^{\alpha} u^{\beta}(-g)^{-1 / 2} \delta_{4}(x-z(\tau)) d \tau,
$$

so that $\tau_{; \beta}^{\alpha \beta}=0$ leads to $u_{; \beta}^{\alpha} u^{\beta}=0$, the geodesic equation. Here, a semicolon indicates covariant differentiation in the background metric. We will keep this notation below: a semicolon will always refer to the metric $g_{\alpha \beta}$, which will always be used to raise and lower Greek indices.

We see that the particle's stress-energy tensor involves the background metric, through the definition of proper time and the factor $(-g)^{-1 / 2}$. If we think of expressing the metric as a formal expansion in powers of $M$, we can similarly expand the particle's stress-energy tensor as 


$$
\tau^{\alpha \beta}=O(m)+O(m M)+O\left(m M^{2}\right)+\cdots .
$$

To keep track of the powers of $m$ and $M$ appearing in various quantities will extremely important in the following discussion. For the weak-field application considered here, we shall truncate Eq. (6.4) after the first two terms.

\section{B. Self-force}

The picture described thus far is that of test mass moving on a geodesic of the background spacetime: the particle has not yet been allowed to alter the spacetime's geometry. We now incorporate this effect by inserting $\tau^{\alpha \beta}$ on the right-hand side of the Einstein field equations. Working linearly in the small mass $m$, we find that this modifies the metric by a term $\gamma_{\alpha \beta}$, so that the total metric is now $g_{\alpha \beta}+\gamma_{\alpha \beta}$. If we impose the Lorentz-gauge condition on $\gamma_{\alpha \beta}$, as we have done in Sec. II B, we find that the metric perturbation is given by Eq. (3.25),

$$
\gamma^{\alpha \beta}(x)=4 \int G_{\mu^{\prime} \nu^{\prime}}^{\alpha \beta}\left(x, x^{\prime}\right) \tau^{\mu^{\prime} \nu^{\prime}}\left(x^{\prime}\right) \sqrt{-g^{\prime}} d^{4} x^{\prime},
$$

where $G_{\mu^{\prime} \nu^{\prime}}^{\alpha \beta}\left(x, x^{\prime}\right)$ is the gravitational Green's function whose "trace-reversed" counterpart is a solution to Eq. (3.22) - Green's equation for a spacetime containing matter. If we again think of expressing the metric as a formal expansion in powers of $M$, we can schematically write

$$
G_{\mu^{\prime} \nu^{\prime}}^{\alpha \beta}=O(1)+O(M)+O\left(M^{2}\right)+\cdots,
$$

which corresponds to Eq. (3.30). Expanding Eq. (6.5) in a similar way, using Eqs. (6.4) and (6.6), we obtain

$$
\gamma_{\alpha \beta}=O(m)+O(m M)+O\left(m M^{2}\right)+\cdots .
$$

This expansion omits terms of order $m^{2}$ (and higher) that would come from the nonlinearities of the Einstein field equations. We shall systematically omit such terms in future expansions, and consistently work to first order in the small mass $m$.

The gravitational self-force corresponds to the metric perturbation $\gamma_{\alpha \beta}$ acting on the particle. If this were the only metric perturbation to be considered - and we will show below that it is not - this action could be described by the statement that the particle now moves on a geodesic of the full metric $g_{\alpha \beta}+\gamma_{\alpha \beta}$. Working again to linear order in the perturbation, we find that the new equations of motion read

$$
u_{; \nu}^{\mu} u^{\nu}=-\frac{1}{2}\left(\gamma_{\alpha ; \beta}^{\mu}+\gamma_{\beta ; \alpha}^{\mu}-\gamma_{\alpha \beta}^{; \mu}+u^{\mu} \gamma_{\alpha \beta ; \gamma} u^{\gamma}\right) u^{\alpha} u^{\beta},
$$

and the right-hand side can be identified with $f_{\text {self }}^{\mu} / m$, the gravitational self-acceleration. Based on Eq. (6.7), we would naively conclude that $f_{\text {self }}^{\alpha} / m=O(m)+O(m M)+O\left(m M^{2}\right)+\cdots$.

Equation (6.8), however, is not valid as it stands. First, we have already indicated that we must insert a mattermediated force on the right-hand side. Second, we know that $\gamma_{\alpha \beta}$ is singular on the particle's world line, so that the right-hand side of Eq. (6.8) is only formally defined; this expression must be regularized and the divergences must be removed. This renormalization of the self-force was carefully performed by Mino et al. [26] and Quinn \& Wald [27] for the case of vacuum spacetimes. Fortunately, the extension of their work to nonvacuum spacetimes is trivial, and it leads to an identical result. The reason is that the renormalization procedure is based on a local analysis that is sensitive only to the immediate vicinity of the world line. So long as the particle moves in a region of spacetime that is empty of matter, which we assume here, the structure of the divergent terms in Eq. (6.8) is the same whether or not matter is present somewhere in the spacetime. After renormalization, the self-force is found to be given by Eq. (1.3), but it is now expressed in terms of a Green's function that is sensitive to the presence of matter.

We conclude that in a spacetime that contains matter, the gravitational self-force is given by its usual expression (1.3), but that it now involves a Green's function that incorporates information about the matter distribution; this information is encoded in the tensor $S_{\mu \alpha \nu \beta}$ defined by Eq. (2.14).

We note that the renormalization procedure removes the $O(\mathrm{~m})$ part of the self-acceleration, which now admits the expansion

$$
f_{\text {self }}^{\alpha} / m=O(m M)+O\left(m M^{2}\right)+\cdots .
$$

Using this together with Eqs. (6.3) and (6.8), we find that the particle's stress-energy tensor is no longer conserved in the background spacetime. Instead, 


$$
\tau_{; \beta}^{\alpha \beta}=O\left(m^{2} M\right)+O\left(m^{2} M^{2}\right)+\cdots .
$$

This is as it should be, because the motion is no longer geodesic in the background spacetime. In a formal sense, $\tau^{\alpha \beta}$ is now conserved in a spacetime with metric $g_{\alpha \beta}+\gamma_{\alpha \beta}$.

\section{Matter-mediated force}

The existence of additional terms on the right-hand side of Eq. (6.8) follows directly from the fact that the stressenergy tensor $T^{\alpha \beta}[g]$ describing the matter distribution depends on the metric. Because inserting the particle in the spacetime has the effect of shifting the metric from $g_{\alpha \beta}$ to $g_{\alpha \beta}+\gamma_{\alpha \beta}$, there must be a corresponding shift in the stress-energy tensor. Physically, this corresponds to the facts that (i) the particle raises a tide on the star and induces internal motions within the fluid, and (ii) the particle sets the star in a small motion around the system's center of mass. Both effects are incorporated in the shift in the stress-energy tensor; as we shall see, however, the bulk motion of the star is much more important for our purposes. We let

$$
\delta T^{\alpha \beta}=T^{\alpha \beta}[g+\gamma]-T^{\alpha \beta}[g]
$$

be the perturbation of the star's stress-energy tensor created by the presence of the particle. Below we will use $T_{*}^{\alpha \beta} \equiv T^{\alpha \beta}[g+\gamma]$ to designate the perturbed stress-energy tensor of the moving star, and $T^{\alpha \beta} \equiv T^{\alpha \beta}[g]$ to represent the background values - the star at rest. Because $T^{\alpha \beta}=O(M)+O\left(M^{2}\right)+\cdots$ and $\gamma_{\alpha \beta}$ can be expanded as in Eq. (6.7), we have that

$$
\delta T^{\alpha \beta}=O(m M)+O\left(m M^{2}\right)+\cdots .
$$

The perturbation in the star's stress-energy tensor must appear, together with $\tau^{\alpha \beta}$, on the right-hand side of the Einstein field equations. It will contribute an additional metric perturbation $\delta g_{\alpha \beta}$, over and above the original perturbation $\gamma_{\alpha \beta}$ directly associated with the particle. Physically, the new perturbation represents the difference between the gravitational field of the moving star and the background field of the star at rest. Because both $\tau^{\alpha \beta}$ and $\delta T^{\alpha \beta}$ are quantities of the first order in $m$, the (linear) metric perturbations can simply be added; the full metric is therefore $g_{\alpha \beta}+\delta g_{\alpha \beta}+\gamma_{\alpha \beta}$.

Imposing the Lorentz-gauge condition separately on both $\delta g_{\alpha \beta}$ and $\gamma_{\alpha \beta}$, we have that

$$
\delta g^{\alpha \beta}(x)=4 \int G_{\mu^{\prime} \nu^{\prime}}^{\alpha \beta}\left(x, x^{\prime}\right) \delta T^{\mu^{\prime} \nu^{\prime}}\left(x^{\prime}\right) \sqrt{-g^{\prime}} d^{4} x^{\prime} .
$$

Using the expansions (6.6) and (6.12), we see that Eq. (6.13) implies

$$
\delta g_{\alpha \beta}=O(m M)+O\left(m M^{2}\right)+\cdots .
$$

The matter-mediated force corresponds to $\delta g_{\alpha \beta}$ acting on the particle. In the absence of $\gamma_{\alpha \beta}$, this action can be described by the statement that the particle must move on a geodesic of the metric $g_{\alpha \beta}^{*} \equiv g_{\alpha \beta}+\delta g_{\alpha \beta}$. Physically, this means that the particle is now subjected to the gravitational field of the moving star. Mathematically, the equations of motion would take the appearance of Eq. (6.8), but with $\delta g_{\alpha \beta}$ taking the place of $\gamma_{\alpha \beta}$. There is no need to renormalize this equation: $\delta g_{\alpha \beta}$ is smooth on the world line.

Our conclusion at this stage is that in a spacetime that contains matter, the equations of motion of a massive particle are given by Eq. (6.1), with a self-force given by Eq. (1.3) and a matter-mediated force given by

$$
f_{\mathrm{mm}}^{\mu}=-\frac{m}{2}\left(\delta g_{\alpha ; \beta}^{\mu}+\delta g_{\beta ; \alpha}^{\mu}-\delta g_{\alpha \beta} ; \mu+u^{\mu} \delta g_{\alpha \beta ; \gamma} u^{\gamma}\right) u^{\alpha} u^{\beta} .
$$

According to Eq. 6.14),

$$
f_{\mathrm{mm}}^{\alpha} / m=O(m M)+O\left(m M^{2}\right)+\cdots,
$$

which should be compared with Eq. (5.9). We see that in a weak-field approximation, both forces contribute terms of order $O(m M)$ to the particle's acceleration.

We have successfully extended the Mino-Sasaki-Tanaka-Quinn-Wald equations of motion to spacetimes containing matter. We emphasize that this extension does not rely on any weak-field assumption regarding the background spacetime. In the following subsections we compute the matter-mediated force of Eq. (6.15); this discussion will rely on a weak-field approximation. 


\section{Determination of $T_{*}^{\alpha \beta}$}

The matter-mediated force of Eq. (6.15) involves the metric perturbation $\delta g_{\alpha \beta}$ which is computed from $\delta T^{\alpha \beta}$, the perturbation in the star's stress-energy tensor; the conversion is given by Eq. (6.13). We have already introduced the notation $T_{*}^{\alpha \beta}=T^{\alpha \beta}+\delta T^{\alpha \beta}$ for the stress-energy tensor of the moving star.

We now need to gather information about $\delta T^{\alpha \beta}$. Our main source will be the conservation equations for the full stress-energy tensor $T^{\alpha \beta}+\delta T^{\alpha \beta}+\tau^{\alpha \beta}$ in a spacetime with metric $g_{\alpha \beta}+\delta g_{\alpha \beta}+\gamma_{\alpha \beta}$. This metric comes with a connection $\Gamma_{\alpha \beta}^{\mu}+\delta \Gamma_{\alpha \beta}^{\mu}+C_{\alpha \beta}^{\mu}$, in which $\Gamma_{\alpha \beta}^{\mu}$ are the Christoffel symbols constructed from the background metric $g_{\alpha \beta}, \delta \Gamma_{\alpha \beta}^{\mu}$ the corrections generated by $\delta g_{\alpha \beta}$, and $C_{\alpha \beta}^{\mu}$ those generated by $\gamma_{\alpha \beta}$. For example,

$$
C_{\alpha \beta}^{\mu}=\frac{1}{2}\left(\gamma_{\alpha ; \beta}^{\mu}+\gamma_{\beta ; \alpha}^{\mu}-\gamma_{\alpha \beta}^{; \mu}\right) \text {. }
$$

By virtue of Eqs. (6.7) and (6.14), we have

$$
C_{\alpha \beta}^{\mu}=O(m)+O(m M)+O\left(m M^{2}\right)+\cdots
$$

and

$$
\delta \Gamma_{\alpha \beta}^{\mu}=O(m M)+O\left(m M^{2}\right)+\cdots .
$$

We also have $\Gamma_{\alpha \beta}^{\mu}=O(M)+O\left(M^{2}\right)+\cdots$ and we shall use the expansions of Eqs. (6.4) and (6.12).

The conservation equations can be expressed as

$$
T_{; \beta}^{\alpha \beta}+\tau_{; \beta}^{\alpha \beta}+\delta T_{, \beta}^{\alpha \beta}+2 C_{\mu \beta}^{(\alpha} T^{\beta) \mu}+2 \Gamma_{\mu \beta}^{(\alpha} \delta T^{\beta) \mu}+2 C_{\mu \beta}^{(\alpha}\left(\delta T^{\beta) \mu}+\tau^{\beta) \mu}\right)+2 \delta \Gamma_{\mu \beta}^{(\alpha}\left(T^{\beta) \mu}+\delta T^{\beta) \mu}+\tau^{\beta) \mu}\right)=0 .
$$

The first term vanishes because the background stress-energy tensor is conserved in the background spacetime. The second term can be ignored, because by virtue of Eq. (6.10), it is of second order in $m$. The third of fourth terms have the expansions $O(m M)+O\left(m M^{2}\right)+\cdots$. Among the remaining terms we have (in schematic notation) $\Gamma \delta T=$ $O\left(m M^{2}\right)+\cdots, C \delta T=O\left(m^{2} M\right)+\cdots, C \tau=O\left(m^{2}\right)+O\left(m^{2} M\right)+\cdots, \delta \Gamma T=O\left(m M^{2}\right)+\cdots, \delta \Gamma \delta T=O\left(m^{2} M^{2}\right)+\cdots$, and $\delta \Gamma \tau=O\left(m^{2} M\right)+\cdots$. We simplify Eq. (6.20) by discarding all terms that are second order in $m$. Incorporating at this stage our weak-field approximation, we also neglect terms that are second order in $M$. The remaining terms are all $O(m M)$ and we obtain

$$
\delta T_{, \beta}^{\alpha \beta}+C_{\beta \gamma}^{\alpha} T^{\beta \gamma}+C_{\beta \gamma}^{\beta} T^{\alpha \gamma}=O\left(m M^{2}\right)
$$

This equation (partially) determines $\delta T^{\alpha \beta}$ in terms of known quantities. Because we have only four equations for ten unknowns, Eq. 6.21) cannot be used without first adopting a specific phenomenology for the matter distribution. We shall come back to this point in Sec. VI F.

It is important to notice that because Eq. (6.21) has been truncated to order $m M$, only the $O(m)$ part of $C^{\mu}{ }_{\alpha \beta}$ is involved in the determination of $\delta T^{\alpha \beta}$ - cf. Eq. (6.18). This can be obtained from the $O(m)$ part of $\gamma_{\alpha \beta}-$ cf. Eq. (6.7). By virtue of Eqs. (3.3), (3.30), and (6.5), this is given by

$$
\gamma_{\text {flat }}^{\alpha \beta}(t, \boldsymbol{x})=4 \int \frac{\bar{\tau}^{\alpha \beta}\left(t-\left|\boldsymbol{x}-\boldsymbol{x}^{\prime}\right|, \boldsymbol{x}^{\prime}\right)}{\left|\boldsymbol{x}-\boldsymbol{x}^{\prime}\right|} d^{3} x^{\prime},
$$

where $\bar{\tau}^{\alpha \beta} \equiv \tau^{\alpha \beta}-\frac{1}{2}\left(\eta_{\mu \nu} \tau^{\mu \nu}\right) \eta^{\alpha \beta}$ is the particle's "trace-reversed" stress-energy tensor. The subscript "flat" indicates that the metric perturbation is calculated as if the particle were moving in flat spacetime (on a Newtonian trajectory around the star); the right-hand side of Eq. (6.22) involves only the $O(m)$ part of $\tau^{\alpha \beta}$ - cf. Eq. (6.4).

Another consequence of truncating Eq. 6.21) is that it can be re-expressed as

$$
T_{*, \beta}^{\alpha \beta}+C_{\beta \gamma}^{\alpha} T_{*}^{\beta \gamma}+C_{\beta \gamma}^{\beta} T_{*}^{\alpha \gamma}=O\left(m M^{2}\right)
$$

in terms of the star's perturbed stress-energy tensor: $T_{*}^{\alpha \beta}=T^{\alpha \beta}+\delta T^{\alpha \beta}$. Here we use the facts that (i) $T^{\alpha \beta}{ }_{, \beta}$ is actually zero for the weak-field spacetimes considered in this paper $-T_{; \beta}^{\alpha \beta}$ is nonzero but is a quantity of order $M^{2}$ 
— and (ii) the difference between $C T_{*}$ and $C T$ is of second order in $m$. Equation (6.23) implies that $T_{*}^{\alpha \beta}$ is conserved in a spacetime with metric $g_{\alpha \beta}^{\text {particle }} \equiv \eta_{\alpha \beta}+\gamma_{\alpha \beta}^{\text {flat }}$, up to terms of order $m M^{2}$. We will refer to this observation below.

Let us now take stock. In order to compute the matter-mediated force of Eq. (6.15) we must first calculate $\delta g_{\alpha \beta}$ using Eq. (6.13). We therefore need to find $\delta T^{\alpha \beta}$, the perturbation of the stress-energy tensor associated with the star's motion around the center of mass. For this we shall use Eq. (6.21), after adopting a specific phenomenology for the matter distribution (Sec. VI F). But Eq. (6.21) involves also the connection coefficients $C_{\alpha \beta}^{\mu}$ computed from

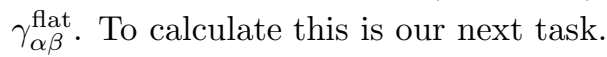

\section{E. Computation of $\gamma_{\alpha \beta}^{\text {flat }}$}

The stress-energy tensor of the particle is given by Eq. 6.2). For the purpose of computing $\gamma_{\alpha \beta}^{\text {flat }}$ we let the particle move in flat spacetime, so that the metric used in Eq. (6.2) to define proper time $\tau$ and the determinant $g$ is $\eta_{\alpha \beta}$, the metric of flat spacetime. After changing the variable of integration to $z^{0}$ and integrating over $\delta\left(t-z_{0}\right)$, we obtain

$$
\tau^{\alpha \beta}(t, \boldsymbol{x})=m(d t / d \tau) v^{\alpha} v^{\beta} \delta_{3}(\boldsymbol{x}-\boldsymbol{z}),
$$

in which $\boldsymbol{z}(t)$ represents the trajectory of the particle, $v^{\alpha}=(1, \boldsymbol{v})$ with $\boldsymbol{v}(t)=d \boldsymbol{z} / d t$, and $d t / d \tau=\left(1-\boldsymbol{v}^{2}\right)^{-1 / 2}$. We recall that although the calculation is carried out in flat spacetime, the particle is actually moving on a geodesic of $g_{\alpha \beta}$; its motion is governed by Newton's equation,

$$
\frac{d \boldsymbol{v}}{d t}=\boldsymbol{g}=-\nabla \Phi
$$

where the right-hand side is evaluated at $\boldsymbol{x}=\boldsymbol{z}(t)$.

It will prove sufficient for our purposes to evaluate $\tau^{\alpha \beta}$ to second order in $\boldsymbol{v}$, the particle's velocity. After trace reversal, we obtain

$$
\begin{aligned}
\bar{\tau}^{00} & =\frac{m}{2}\left[1+\frac{3}{2} \boldsymbol{v}^{2}+O\left(v^{4}\right)\right] \delta_{3}(\boldsymbol{x}-\boldsymbol{z}), \\
\bar{\tau}^{0 a} & =m v^{a}\left[1+O\left(v^{2}\right)\right] \delta_{3}(\boldsymbol{x}-\boldsymbol{z}), \\
\bar{\tau}^{a b} & =\frac{m}{2}\left\{\delta^{a b}\left[1-\frac{1}{2} \boldsymbol{v}^{2}+O\left(v^{4}\right)\right]+2 v^{a} v^{b}\left[1+O\left(v^{2}\right)\right]\right\} \delta_{3}(\boldsymbol{x}-\boldsymbol{z}) .
\end{aligned}
$$

To be consistent we also Taylor-expand the right-hand side of Eq. (6.22) about the current time $t$. This gives

$$
\gamma_{\text {flat }}^{\alpha \beta}(t, \boldsymbol{x})=4 \int \frac{\bar{\tau}^{\alpha \beta}\left(t, \boldsymbol{x}^{\prime}\right)}{\left|\boldsymbol{x}-\boldsymbol{x}^{\prime}\right|} d^{3} x^{\prime}-4 \frac{\partial}{\partial t} \int \bar{\tau}^{\alpha \beta}\left(t, \boldsymbol{x}^{\prime}\right) d^{3} x^{\prime}+2 \frac{\partial^{2}}{\partial t^{2}} \int \bar{\tau}^{\alpha \beta}\left(t, \boldsymbol{x}^{\prime}\right)\left|\boldsymbol{x}-\boldsymbol{x}^{\prime}\right| d^{3} x^{\prime}+\cdots .
$$

It is now straightforward to substitute Eqs. 6.26) into Eq. (6.27) and perform the integrations. For example,

$$
\gamma_{\text {flat }}^{00}=\frac{2 m \lambda}{|\boldsymbol{x}-\boldsymbol{z}|}-2 m \frac{\partial \lambda}{\partial t}+m \frac{\partial^{2}}{\partial t^{2}} \lambda|\boldsymbol{x}-\boldsymbol{z}|+\cdots
$$

where $\lambda=1+\frac{3}{2} \boldsymbol{v}^{2}+O\left(v^{4}\right)$. This result simplifies considerably: When the time derivative acts on $\boldsymbol{v}$, it generates via Eq. (6.25) a term of order $g \sim M /|\boldsymbol{z}|^{2}$ that can be neglected, because we are interested only in the $O(m)$ part of $\gamma_{\alpha \beta}$. We are therefore left with evaluating $\partial^{2}|\boldsymbol{x}-\boldsymbol{z}| / \partial t^{2}$, which is a simple task. Subjecting the other components to similar manipulations, we arrive at

$$
\begin{aligned}
& \gamma_{\text {flat }}^{00}(t, \boldsymbol{x})=\frac{2 m}{|\boldsymbol{x}-\boldsymbol{z}|}\left[1+2 \boldsymbol{v}^{2}-\frac{1}{2}(\hat{\boldsymbol{n}} \cdot \boldsymbol{v})^{2}+O\left(v^{4}\right)\right]+O(m M), \\
& \gamma_{\text {flat }}^{0 a}(t, \boldsymbol{x})=\frac{4 m v^{a}}{|\boldsymbol{x}-\boldsymbol{z}|}\left[1+O\left(v^{2}\right)\right]+O(m M), \\
& \gamma_{\text {flat }}^{a b}(t, \boldsymbol{x})=\frac{2 m}{|\boldsymbol{x}-\boldsymbol{z}|}\left\{\delta^{a b}\left[1-\frac{1}{2}(\hat{\boldsymbol{n}} \cdot \boldsymbol{v})^{2}\right]+2 v^{a} v^{b}+O\left(v^{4}\right)\right\}+O(m M),
\end{aligned}
$$

where $\hat{\boldsymbol{n}}=(\boldsymbol{x}-\boldsymbol{z}) /|\boldsymbol{x}-\boldsymbol{z}|$. 


\section{F. Determination of $T_{*}^{\alpha \beta}$ (continued)}

As we have remarked at the end of Sec. VI D, the determination of $T_{*}^{\alpha \beta}$ via the conservation equation (6.23) is possible only if we adopt a specific phenomenology for the matter distribution. We shall make the simplest choice, and describe the star as a particle of mass $M$ moving on a trajectory $\boldsymbol{Z}(t)$ with a velocity $\boldsymbol{V}(t)=d \boldsymbol{Z} / d t$. The pointlike nature of the moving star will not be a problem here: First, for the purposes of this calculation the star is not subjected to its own field because, as we have pointed out, it moves in a spacetime with metric $g_{\alpha \beta}^{\text {particle }}=\eta_{\alpha \beta}+\gamma_{\alpha \beta}^{\text {flat }}$; second, the singularity in $\delta g_{\alpha \beta}$ is far removed from the particle's world line and is therefore not an obstacle in the calculation of the matter-mediated force of Eq. (6.15). Notice that in the following developments we shall formally treat both $\boldsymbol{Z}$ and $\boldsymbol{V}$ as quantities of the first order in $m$.

Following the steps leading to Eq. (6.24), we find that the stress-energy tensor of the moving star takes the form

$$
T_{*}^{\alpha \beta}(t, \boldsymbol{x})=M \frac{d t / d \tau}{\sqrt{-g_{\text {particle }}}} V^{\alpha} V^{\beta} \delta_{3}(\boldsymbol{x}-\boldsymbol{Z}),
$$

where $V^{\alpha}=(1, \boldsymbol{V})$ and $d t / d \tau=\left(-g_{\mu \nu}^{\text {particle }} V^{\mu} V^{\nu}\right)^{-1 / 2}$. After discarding terms of order $m^{2}$, this reduces to $T_{*}^{00}=$ $M \delta_{3}(\boldsymbol{x}-\boldsymbol{Z})+M\left(\gamma_{\text {flat }}^{00}-\frac{1}{2} \delta_{a b} \gamma_{\text {flat }}^{a b}\right) \delta_{3}(\boldsymbol{x}), T_{*}^{0 a}=M V^{a} \delta_{3}(\boldsymbol{x})$, and $T_{*}^{a b}=O\left(m^{2} M\right)$. The perturbation $\delta T^{\alpha \beta}$ can be obtained from these results by removing the background values, $T^{00}=M \delta_{3}(\boldsymbol{x})$ and $T^{0 a}=T^{a b}=0$.

Substitution of our expressions for $\delta T^{\alpha \beta}$ into Eq. (6.21) leads to equations of motion for the star. The spatial components of the conservation equation imply

$$
\frac{d V^{a}}{d t}=\frac{1}{2} \frac{\partial}{\partial x^{a}} \gamma_{\text {flat }}^{00}+\frac{\partial}{\partial t} \gamma_{\text {flat }}^{0 a}+O(m M)
$$

in which the right-hand side is evaluated at $\boldsymbol{x}=0$. We find that the time component of the conservation equation holds as an identity, but this confirms the necessity of including $\sqrt{-g_{\text {particle }}}$ in our expression for the stress-energy tensor.

It is a simple task to evaluate the partial derivatives of Eq. (6.31) starting from the expressions listed in Eq. (6.29); in this procedure we use Eq. (6.25) to dismiss $d \boldsymbol{v} / d t$ as a term of order $M /|\boldsymbol{z}|^{2}$. After evaluating the results at $\boldsymbol{x}=0$, we obtain

$$
\frac{d \boldsymbol{V}}{d t}=\frac{m}{|\boldsymbol{z}|^{2}}\left\{\left[1+2 \boldsymbol{v}^{2}-\frac{3}{2}(\hat{\boldsymbol{z}} \cdot \boldsymbol{v})^{2}+O\left(v^{4}\right)\right] \hat{\boldsymbol{z}}-3(\hat{\boldsymbol{z}} \cdot \boldsymbol{v}) \boldsymbol{v}\right\}+O(m M),
$$

where $\hat{\boldsymbol{z}}=\boldsymbol{z} /|\boldsymbol{z}|$. The motion of the star is now determined to a degree of accuracy sufficient for our remaining calculations. Equation (6.32) confirms that $\boldsymbol{V}$, and therefore $\boldsymbol{Z}$, are to be treated as quantities of order $m$. We note that in the present context in which the star has become a point mass, the motion of the particle is still governed by Eq. (6.25), but that the gravitational field $\boldsymbol{g}$ now takes the explicit form

$$
\boldsymbol{g}=-\frac{M}{|\boldsymbol{z}|^{2}} \hat{z}
$$

Equation (6.25) determines the vectors $\boldsymbol{z}(t)$ and $\boldsymbol{v}(t)=d \boldsymbol{z} / d t$ that appear in Eq. 6.32).

The perturbed stress-energy tensor of the moving star is now completely determined. Its final expression is obtained by substituting the potentials of Eq. (6.29) into the results given before. This yields

$$
\begin{aligned}
& T_{*}^{00}(t, \boldsymbol{x})=M \delta_{3}(\boldsymbol{x}-\boldsymbol{Z})-\frac{m M}{|\boldsymbol{z}|}\left[1-2 \boldsymbol{v}^{2}-\frac{1}{2}(\hat{\boldsymbol{z}} \cdot \boldsymbol{v})^{2}+O\left(v^{4}\right)\right] \delta_{3}(\boldsymbol{x})+O\left(m M^{2}\right), \\
& T_{*}^{0 a}(t, \boldsymbol{x})=M V^{a} \delta_{3}(\boldsymbol{x}),
\end{aligned}
$$

and $T_{*}^{a b}=O\left(m^{2} M\right)$.

\section{G. Computation of $g_{\alpha \beta}^{*}$}

The metric $g_{\alpha \beta}^{*}=g_{\alpha \beta}+\delta g_{\alpha \beta}$ was introduced in Sec. VI C, in the paragraph following Eq. (6.14). It represents the gravitational field of the moving star, and the action of the matter-mediated force of Eq. (6.15) can be described 
by the statement that the particle must move on a geodesic of this metric. In the next subsection we will use this statement to produce the equations of motion of the particle under the sole action of the matter-mediated force. In this subsection we calculate $g_{\alpha \beta}^{*}$.

The metric of the moving star differs from $g_{\alpha \beta}$, the metric of the star at rest, by $\delta g_{\alpha \beta}$, which can be computed from Eq. (6.13). Keeping only the $O(m M)$ part of this equation, which involves the $O(1)$ part of the gravitational Green's function - cf. Eqs. (3.3) and (3.30), we write this as

$$
\delta g^{\alpha \beta}(t, \boldsymbol{x})=4 \int \frac{\delta \bar{T}^{\alpha \beta}\left(t-\left|\boldsymbol{x}-\boldsymbol{x}^{\prime}\right|, \boldsymbol{x}^{\prime}\right)}{\left|\boldsymbol{x}-\boldsymbol{x}^{\prime}\right|} d^{3} x^{\prime}+O\left(m M^{2}\right),
$$

where $\delta \bar{T}^{\alpha \beta}=\delta T^{\alpha \beta}-\frac{1}{2}\left(\eta_{\mu \nu} \delta T^{\mu \nu}\right) \eta^{\alpha \beta}$. At the same time, we recall from Sec. II A that $g_{\alpha \beta}$ differs from $\eta_{\alpha \beta}$ by a quantity $h_{\alpha \beta} \equiv-2 \Phi \chi_{\alpha \beta}$ that can expressed as

$$
h^{\alpha \beta}(t, \boldsymbol{x})=4 \int \frac{\bar{T}^{\alpha \beta}\left(t-\left|\boldsymbol{x}-\boldsymbol{x}^{\prime}\right|, \boldsymbol{x}^{\prime}\right)}{\left|\boldsymbol{x}-\boldsymbol{x}^{\prime}\right|} d^{3} x^{\prime},
$$

where $\bar{T}^{\alpha \beta}$ is the trace-reversed stress-energy tensor of the background spacetime. Adding Eqs. 6.35) and (6.36), we find that

$$
g_{\alpha \beta}^{*}=\eta_{\alpha \beta}+h_{\alpha \beta}^{*},
$$

where $h_{\alpha \beta}^{*} \equiv h_{\alpha \beta}+\delta g_{\alpha \beta}$ is given by

$$
h_{*}^{\alpha \beta}(t, \boldsymbol{x})=4 \int \frac{\bar{T}_{*}^{\alpha \beta}\left(t-\left|\boldsymbol{x}-\boldsymbol{x}^{\prime}\right|, \boldsymbol{x}^{\prime}\right)}{\left|\boldsymbol{x}-\boldsymbol{x}^{\prime}\right|} d^{3} x^{\prime}+O\left(m M^{2}\right),
$$

and where $\bar{T}_{*}^{\alpha \beta} \equiv T_{*}^{\alpha \beta}-\frac{1}{2}\left(\eta_{\mu \nu} T_{*}^{\mu \nu}\right) \eta^{\alpha \beta}$ can be calculated from Eq. (6.34). To first order in $m$, this gives $\bar{T}_{*}^{00}=\frac{1}{2} T_{*}^{00}$, $\bar{T}_{*}^{0 a}=T_{*}^{0 a}$, and $\bar{T}_{*}^{a b}=\frac{1}{2} \delta^{a b} T_{*}^{00}$.

We shall describe in some detail the computation of $h_{*}^{00}$. We first Taylor-expand $T_{*}^{00}\left(t-\left|\boldsymbol{x}-\boldsymbol{x}^{\prime}\right|, \boldsymbol{x}^{\prime}\right)$ about the current time $t$ and express $h_{*}^{00}$ as

$$
\begin{aligned}
h_{*}^{00}= & 2 \int \frac{T_{*}^{00}\left(t, \boldsymbol{x}^{\prime}\right)}{\left|\boldsymbol{x}-\boldsymbol{x}^{\prime}\right|} d^{3} x^{\prime}-2 \frac{\partial}{\partial t} \int T_{*}^{00}\left(t, \boldsymbol{x}^{\prime}\right) d^{3} x^{\prime}+\frac{\partial^{2}}{\partial t^{2}} \int T_{*}^{00}\left(t, \boldsymbol{x}^{\prime}\right)\left|\boldsymbol{x}-\boldsymbol{x}^{\prime}\right| d^{3} x^{\prime} \\
& -\frac{1}{3} \frac{\partial^{3}}{\partial t^{3}} \int T_{*}^{00}\left(t, \boldsymbol{x}^{\prime}\right)\left|\boldsymbol{x}-\boldsymbol{x}^{\prime}\right|^{2} d^{3} x^{\prime}+\cdots+O\left(m M^{2}\right) .
\end{aligned}
$$

We will see that truncating the series after four terms gives us sufficient accuracy; it is very important, however, to keep the last term involving three time derivatives. After substitution of Eq. (6.34), we obtain

$$
\begin{aligned}
h_{*}^{00}= & \frac{2 M}{|\boldsymbol{x}-\boldsymbol{Z}|}+M \frac{\partial^{2}}{\partial t^{2}}|\boldsymbol{x}-\boldsymbol{Z}|-\frac{1}{3} M \frac{\partial^{3}}{\partial t^{3}}|\boldsymbol{x}-\boldsymbol{Z}|^{2}+\cdots \\
& -\frac{2 m M}{|\boldsymbol{x}|} \frac{\lambda}{|\boldsymbol{z}|}+2 m M \frac{\partial}{\partial t} \frac{\lambda}{|\boldsymbol{z}|}-m M|\boldsymbol{x}| \frac{\partial^{2}}{\partial t^{2}} \frac{\lambda}{|\boldsymbol{z}|}+\frac{1}{3} m M|\boldsymbol{x}|^{2} \frac{\partial^{3}}{\partial t^{3}} \frac{\lambda}{|\boldsymbol{z}|}+\cdots+O\left(m M^{2}\right),
\end{aligned}
$$

where $\lambda=1-2 \boldsymbol{v}^{2}-\frac{1}{2}(\hat{\boldsymbol{z}} \cdot \boldsymbol{v})^{2}+O\left(v^{4}\right)$. It is a straightforward task to evaluate the time derivatives contained in Eq. (6.40); for this we involve Eq. (6.32) and we make sure to discard all terms that are not first-order in both $m$ and $M$. For the first line (FL) on the right-hand side of Eq. (6.40) we obtain

$$
\mathrm{FL}=\frac{2 M}{|\boldsymbol{x}-\boldsymbol{Z}|}-m M \frac{\boldsymbol{x} \cdot \boldsymbol{z}}{|\boldsymbol{x}||\boldsymbol{z}|^{3}}\left[1+O\left(v^{2}\right)\right]-\frac{2}{3} m M \boldsymbol{x} \cdot \frac{d \boldsymbol{g}}{d t}\left[1+O\left(v^{2}\right)\right]+O\left(m M^{2}\right),
$$

where $\boldsymbol{g}$ is defined by Eq. (6.33). In Eq. (6.40), the first set of "..." terms represent a correction of order $v^{4}$ to the second term of Eq. (6.41); these can be safely ignored. The last member of Eq. (6.41) comes from the third-derivative term in Eq. (6.40); this will be seen to give rise to a radiation-damping term in the matter-mediated force. For the second line (SL) on the right-hand side of Eq. (6.40) we find

$$
\mathrm{SL}=-\frac{2 m M}{|\boldsymbol{x}||\boldsymbol{z}|}\left[1+O\left(v^{2}\right)\right]-2 m M \frac{\boldsymbol{z} \cdot \boldsymbol{v}}{|\boldsymbol{z}|^{3}}\left[1+O\left(v^{2}\right)\right]+O\left(m M^{2}\right) .
$$


Here the third-derivative term in Eq. (6.40) is seen to give rise to the $O\left(v^{2}\right)$ correction in the second member of Eq. (6.42), and the "..." terms are responsible for $O\left(v^{4}\right)$ corrections in the first member.

Gathering the results, we arrive at

$$
h_{*}^{00}(t, \boldsymbol{x})=\frac{2 M}{|\boldsymbol{x}-\boldsymbol{Z}|}-\frac{m M}{|\boldsymbol{x}||\boldsymbol{z}|}\left(2+\frac{\boldsymbol{x} \cdot \boldsymbol{z}}{|\boldsymbol{z}|^{2}}\right)-\frac{2}{3} m \boldsymbol{x} \cdot \frac{d \boldsymbol{g}}{d t}-2 m M \frac{\boldsymbol{z} \cdot \boldsymbol{v}}{|\boldsymbol{z}|^{3}}+O\left(2 \mathrm{PN}, m M^{2}\right),
$$

in which $O(2 \mathrm{PN})$ regroups all the $O\left(v^{2}\right)$ correction terms displayed in Eqs. (6.41) and (6.42). We note that on the right-hand side of Eq. (6.43), the first member is of order $\Phi$ and is therefore designated as "Newtonian". The second member is of order $m /|\boldsymbol{z}|$ relative to $\Phi$, and it represents a post-Newtonian (1PN) correction [52]. Because they involve an additional power of $\boldsymbol{v}$, the last two members are 1.5PN corrections.

The calculation of $h_{*}^{0 a}$ involves similar steps, but the computations are substantially simpler. We simply quote the result:

$$
h_{*}^{0 a}(t, \boldsymbol{x})=\frac{4 M V^{a}}{|\boldsymbol{x}|}+4 m g^{a}+O\left(1.5 \mathrm{PN}, m M^{2}\right) .
$$

Relative to the leading, "Newtonian" term in $h_{*}^{00}$, the first term on the right-hand side of Eq. (6.44) represents a $0.5 \mathrm{PN}$ correction, while the second term is a $1 \mathrm{PN}$ correction. Because $h_{*}^{0 a}$ couples to the particle's velocity $v^{a}$, these post-Newtonian labels should actually be promoted to $1 \mathrm{PN}$ and $1.5 \mathrm{PN}$, respectively; the error term is then $2 \mathrm{PN}$ and $h_{*}^{0 a}$ has been computed to a sufficient degree of accuracy.

The metric of the moving star is now completely determined. We have, from Eq. (6.37),

$$
g_{00}^{*}=-\left(1-h_{*}^{00}\right)+O\left(m M^{2}\right), \quad g_{0 a}^{*}=-h_{*}^{0 a}+O\left(m M^{2}\right), \quad g_{a b}^{*}=\delta_{a b}\left(1+h_{*}^{00}\right)+O\left(m M^{2}\right),
$$

with $h_{*}^{00}$ and $h_{*}^{0 a}$ given by Eqs. (6.43) and (6.44), respectively. In those expressions, $\boldsymbol{z}(t)$ represents the Newtonian motion of the particle around the fixed star, $\boldsymbol{v}(t)=d \boldsymbol{z} / d t$, and $d \boldsymbol{v} / d t=\boldsymbol{g}=-M \boldsymbol{z} /|\boldsymbol{z}|^{3}$. On the other hand, $\boldsymbol{Z}(t)$ represents the order- $m$ motion of the star in the gravitational field of the particle, $\boldsymbol{V}(t)=d \boldsymbol{Z} / d t$, and $d \boldsymbol{V} / d t$ is determined by Eq. (6.32).

\section{H. Motion of the particle under the matter-mediated force}

In this subsection we calculate the motion of the particle under the sole action of the matter-mediated force; we neglect for now the action of the self-force, which will be incorporated later. In this subsection, therefore, we shall write the equations of motion as $u_{; \beta}^{\alpha} u^{\beta}=f_{\mathrm{mm}}^{\alpha}$. The force could be calculated directly from Eq. (6.15) by substituting $\delta g_{\alpha \beta}$ obtained from Eq. (6.45). It is easier, however, to proceed in the following way. First, we recognize that the equations of motion are equivalent to the statement that the particle moves on a geodesic of the metric $g_{\alpha \beta}^{*}=g_{\alpha \beta}+\delta g_{\alpha \beta}$. Second, we generate the equations of motion by constructing a suitable Lagrangian function $L(\boldsymbol{x}, \dot{\boldsymbol{x}})$ which we then substitute into the Euler-Lagrange equations. The practical advantage of this method is that it allows us to straightforwardly switch from a world line parameterized by $\tau$, the particle's proper time on the background spacetime, to one parameterized by $t$, the time coordinate. In the following we will denote the updated trajectory of the particle by $\boldsymbol{x}(t)$ and its updated velocity by $\dot{\boldsymbol{x}}(t)=d \boldsymbol{x} / d t$. The Newtonian values will still be denoted $\boldsymbol{z}(t)$ and $\boldsymbol{v}(t)$. Because the matter-mediated force provides a correction of order $m$ to the Newtonian motion, we have $\boldsymbol{x}(t)=\boldsymbol{z}(t)+O(m)$ and $\dot{\boldsymbol{x}}(t)=\boldsymbol{v}(t)+O(m)$. In the next subsection we will abolish this distinction of notation and use $\boldsymbol{z}$ and $\boldsymbol{v}$ to refer to the updated trajectory.

We take the Lagrangian function to be

$$
L(\boldsymbol{x}, \dot{\boldsymbol{x}})=1-\sqrt{-g_{\alpha \beta}^{*} \dot{x}^{\alpha} \dot{x}^{\beta}},
$$

where $\dot{x}^{\alpha}=(1, \dot{\boldsymbol{x}})$; as we have indicated, we use $t$ as a parameter on the world line. We substitute the metric of Eq. (6.45) into Eq. (6.46), and to simplify we expand the square root to second post-Newtonian (2PN) order; in this procedure we also discard terms that are second order in $M$. The result is

$$
L=\frac{1}{2} \dot{\boldsymbol{x}}^{2}+\frac{1}{2} h_{*}^{00}+\frac{1}{8} \dot{\boldsymbol{x}}^{4}-h_{*}^{0 a} \dot{x}^{a}+\frac{3}{4} h_{*}^{00} \dot{\boldsymbol{x}}^{2}+O\left(2 \mathrm{PN}, M^{2}\right) .
$$

After substitution of Eqs. (6.43) and (6.44), we notice that the Lagrangian contains a term $-m M \boldsymbol{z} \cdot \boldsymbol{v} /|\boldsymbol{z}|^{3}$. This is a function of time only which does not affect the equations of motion; we therefore remove it from the Lagrangian. 
The Lagrangian also contains a term $-4 m \dot{\boldsymbol{x}} \cdot \boldsymbol{g}$. This differs from $4 m \boldsymbol{x} \cdot d \boldsymbol{g} / d t$ by a total derivative $-4 m d(\boldsymbol{x} \cdot \boldsymbol{g}) / d t$ that can also be deleted from the Lagrangian. The final result is an effective Lagrangian

$$
L_{\mathrm{eff}}=\frac{1}{2} \dot{\boldsymbol{x}}^{2}+\frac{M}{|\boldsymbol{x}-\boldsymbol{Z}|}\left(1+\frac{3}{2} \dot{\boldsymbol{x}}^{2}\right)+\frac{1}{8} \dot{\boldsymbol{x}}^{4}-\frac{m M}{|\boldsymbol{x}||\boldsymbol{z}|}\left(1+\frac{\boldsymbol{x} \cdot \boldsymbol{z}}{2|\boldsymbol{z}|^{2}}\right)-\frac{4 M}{|\boldsymbol{x}|} \dot{\boldsymbol{x}} \cdot \boldsymbol{V}+\frac{11}{3} m \boldsymbol{x} \cdot \frac{d \boldsymbol{g}}{d t}+O\left(2 \mathrm{PN}, M^{2}\right)
$$

that is ready to be substituted into the Euler-Lagrange equations.

The first member of the Euler-Lagrange equation is

$$
\frac{d}{d t} \frac{\partial L_{\mathrm{eff}}}{\partial \dot{x}^{a}}=\Lambda^{a b} \frac{d \dot{x}^{b}}{d t}-\frac{3 M}{|\boldsymbol{x}-\boldsymbol{Z}|}(\boldsymbol{x} \cdot \dot{\boldsymbol{x}}) \dot{x}^{a}+\frac{3 M}{|\boldsymbol{x}|^{3}}(\boldsymbol{x} \cdot \boldsymbol{V}+\dot{\boldsymbol{x}} \cdot \boldsymbol{Z}) \dot{x}^{a}-\frac{4 m M}{|\boldsymbol{x}||\boldsymbol{z}|^{3}} z^{a}+\frac{4 M}{|\boldsymbol{x}|^{3}}(\boldsymbol{x} \cdot \dot{\boldsymbol{x}}) V^{a},
$$

where $\Lambda^{a b}=\left(1+\frac{1}{2} \dot{\boldsymbol{x}}^{2}+3 M /|\boldsymbol{x}-\boldsymbol{Z}|\right) \delta^{a b}+\dot{x}^{a} \dot{x}^{b}$. To obtain this we have used Eq. (6.32), appropriately truncated at Newtonian order, to evaluate $d V^{a} / d t$. The second member is

$$
\frac{\partial L_{\mathrm{eff}}}{\partial x^{a}}=-M \frac{x^{a}-Z^{a}}{|\boldsymbol{x}-\boldsymbol{Z}|^{3}}\left(1+\frac{3}{2} \dot{\boldsymbol{x}}^{2}\right)+\frac{m M}{|\boldsymbol{x}|^{3}|\boldsymbol{z}|}\left(1+\frac{\boldsymbol{x} \cdot \boldsymbol{z}}{2|\boldsymbol{z}|^{2}}\right) x^{a}-\frac{m M}{2|\boldsymbol{x}||\boldsymbol{z}|^{3}} z^{a}+\frac{4 M}{|\boldsymbol{x}|^{3}}(\dot{\boldsymbol{x}} \cdot \boldsymbol{V}) x^{a}+\frac{11}{3} m \frac{d g^{a}}{d t} .
$$

The equations of motion are obtained by equating Eq. (6.49) to Eq. (6.50) and solving for $d \dot{x}^{a} / d t$. This requires the inversion of $\Lambda^{a b}$, which can be performed approximately to first post-Newtonian order. To simplify our expressions we replace $z^{a}$ by $x^{a}+O(m)$ in the terms proportional to $m M$, and we also replace $\boldsymbol{x}$ by $\boldsymbol{x}-\boldsymbol{Z}=\boldsymbol{x}+O(m)$ in terms that are already linear in $m$. (It is of course important that we make these substitutions after varying the Lagrangian, and not before.)

The final result is

$$
\begin{aligned}
\frac{d \dot{x}^{a}}{d t}= & -\frac{M}{|\boldsymbol{x}-\boldsymbol{Z}|^{3}}\left[\left(1+\dot{\boldsymbol{x}}^{2}-\frac{5 m}{|\boldsymbol{x}-\boldsymbol{Z}|}-4 \dot{\boldsymbol{x}} \cdot \boldsymbol{V}\right)\left(x^{a}-Z^{a}\right)-(\boldsymbol{x}-\boldsymbol{Z}) \cdot(4 \dot{\boldsymbol{x}}-3 \boldsymbol{V}) \dot{x}^{a}+4(\boldsymbol{x}-\boldsymbol{Z}) \cdot \dot{\boldsymbol{x}} V^{a}\right] \\
& +\frac{11}{3} m \frac{d g^{a}}{d t}+O\left(2 \mathrm{PN}, M^{2}\right) .
\end{aligned}
$$

We recall that $\boldsymbol{Z}(t)=O(m)$ represents the trajectory of the moving star, that $\boldsymbol{V}(t)=d \boldsymbol{Z} / d t$ is determined by Eq. (6.32), and that $\boldsymbol{g}$, given by Eq. (6.33), is the Newtonian gravitational field of the star at rest. Equation (6.51) governs the motion of the particle under the sole action of the matter-mediated force. We see that the last term, which represents a $1.5 \mathrm{PN}$ correction to the Newtonian equations of motion, gives rise to radiation damping. This term, however, is precisely canceled out by the gravitational self-force of Eq. (5.31). We conclude that the motion of the particle, under the combined action of the self-force and the matter-mediated force, is conservative at this level of approximation.

\section{Summary and comparison with post-Newtonian theory}

This is the end of the line, and we better summarize. A particle of mass $m$ moves in the gravitational field of a star of mass $M$. The particle's trajectory is denoted $\boldsymbol{z}(t)$ and its velocity is $\boldsymbol{v}(t)=d \boldsymbol{z} / d t$. The star is also moving, on a trajectory $\boldsymbol{Z}(t)$ with a velocity $\boldsymbol{V}(t)=d \boldsymbol{Z} / d t$. We let $\boldsymbol{\rho}=\boldsymbol{z}-\boldsymbol{Z}$ be the separation between the two objects, and we use the notation $\rho=|\boldsymbol{z}-\boldsymbol{Z}|$. We let $\boldsymbol{g}=-M \boldsymbol{z} /|\boldsymbol{z}|^{3}$ be the Newtonian gravitational field of the star at rest, $\boldsymbol{g}^{*}=-M \boldsymbol{\rho} / \rho^{3}$ is the gravitational field of the moving star, and $\delta \boldsymbol{g}=\boldsymbol{g}^{*}-\boldsymbol{g}$ is the difference between the two. The spatial components of Eq. (6.1) then give

$$
\frac{d \boldsymbol{v}}{d t}-\boldsymbol{g}=\frac{\boldsymbol{f}_{\text {self }}}{m}+\frac{\boldsymbol{f}_{\mathrm{mm}}}{m}
$$

for the particle's equations of motion. The gravitational self-force was computed in Sec. V C and is given by

$$
\frac{\boldsymbol{f}_{\text {self }}}{m}=-\frac{11}{3} m \frac{d \boldsymbol{g}}{d t}+O\left(2 \mathrm{PN}, M^{2}\right) .
$$

The matter-mediated force was computed in the preceding subsection and is given by

$$
\frac{\boldsymbol{f}_{\mathrm{mm}}}{m}=\delta \boldsymbol{g}+\boldsymbol{g}^{*}\left(\boldsymbol{v}^{2}-\frac{5 m}{\rho}-4 \boldsymbol{v} \cdot \boldsymbol{V}\right)+\frac{M}{\rho^{3}}[\boldsymbol{\rho} \cdot(4 \boldsymbol{v}-3 \boldsymbol{V}) \boldsymbol{v}-4(\boldsymbol{\rho} \cdot \boldsymbol{v}) \boldsymbol{V}]+\frac{11}{3} m \frac{d \boldsymbol{g}}{d t}+O\left(2 \mathrm{PN}, M^{2}\right) .
$$


The final form of the equations of motion is obtained by combining Eqs. (6.52)-(6.54). The result is

$$
\frac{d \boldsymbol{v}}{d t}=-\frac{M}{\rho^{3}}\left[\left(1+\boldsymbol{v}^{2}-\frac{5 m}{\rho}-4 \boldsymbol{v} \cdot \boldsymbol{V}\right) \boldsymbol{\rho}-\boldsymbol{\rho} \cdot(4 \boldsymbol{v}-3 \boldsymbol{V}) \boldsymbol{v}+4(\boldsymbol{\rho} \cdot \boldsymbol{v}) \boldsymbol{V}\right]+O\left(2 \mathrm{PN}, M^{2}\right),
$$

and this contains no trace of a radiation-reaction force. The motion of the star, on the other hand, is governed by Eq. (6.32), which we rewrite as

$$
\frac{d \boldsymbol{V}}{d t}=\frac{m}{\rho^{3}}\left\{\left[1+2 \boldsymbol{v}^{2}-\frac{3}{2}\left(\frac{\boldsymbol{\rho} \cdot \boldsymbol{v}}{\rho}\right)^{2}\right] \boldsymbol{\rho}-3(\boldsymbol{\rho} \cdot \boldsymbol{v}) \boldsymbol{v}\right\}+O(2 \mathrm{PN}, m M) .
$$

In going from Eq. (6.32) to Eq. (6.56) we have allowed for the fact that $\boldsymbol{\rho} \equiv \boldsymbol{z}-\boldsymbol{Z}=\boldsymbol{z}+O(\mathrm{~m})$.

The reader familiar with post-Newtonian theory (as presented, for example, in Ref. [60]) will have undoubtedly noticed a similarity between the calculations presented here and the standard post-Newtonian treatment of a system of point masses. While similarities are surely present, the differences are important. The most noticeable difference is concerned with self-field effects, which are sometimes swept under the rug in the post-Newtonian treatment - the mass of each body is simply renormalized every time an infinite self-field appears in the equations. By contrast, the computations presented in this section are completely free of infinities - those have been taken care of, once and for all, during the construction of the self-force.

Equations (6.55) and (6.56) should be compared with the equations derived from a standard post-Newtonian treatment - the Einstein-Infeld-Hoffmann equations of motion [1]. These are 54

$$
\frac{d \boldsymbol{v}}{d t}=-\frac{M}{\rho^{3}}\left\{\left[1-\frac{4 M}{\rho}-\frac{5 m}{\rho}+\boldsymbol{v}^{2}+2 \boldsymbol{V}^{2}-4 \boldsymbol{v} \cdot \boldsymbol{V}-\frac{3}{2}\left(\frac{\boldsymbol{\rho} \cdot \boldsymbol{V}}{\rho}\right)^{2}\right] \boldsymbol{\rho}-\boldsymbol{\rho} \cdot(4 \boldsymbol{v}-3 \boldsymbol{V})(\boldsymbol{v}-\boldsymbol{V})\right\}+O(2 \mathrm{PN})
$$

and

$$
\frac{d \boldsymbol{V}}{d t}=\frac{m}{\rho^{3}}\left\{\left[1-\frac{4 m}{\rho}-\frac{5 M}{\rho}+\boldsymbol{V}^{2}+2 \boldsymbol{v}^{2}-4 \boldsymbol{v} \cdot \boldsymbol{V}-\frac{3}{2}\left(\frac{\boldsymbol{\rho} \cdot \boldsymbol{v}}{\rho}\right)^{2}\right] \boldsymbol{\rho}+\boldsymbol{\rho} \cdot(4 \boldsymbol{V}-3 \boldsymbol{v})(\boldsymbol{v}-\boldsymbol{V})\right\}+O(2 \mathrm{PN}) .
$$

If we eliminate all terms of order $m^{2}$ and $M^{2}$ from Eq. (6.57), we recover Eq. (6.55). If, on the other hand, we remove all terms of order $m^{2}$ and $m M$ from Eq. (6.58), we recover Eq. (6.56). Our results are therefore perfectly consistent with the standard post-Newtonian treatment.

\section{ACKNOWLEDGMENTS}

The work presented here was supported by the Natural Sciences and Engineering Research Council of Canada. We are grateful to Leor Barack, Eanna Flanagan, Valeri Frolov, Amos Ori, Misao Sasaki, Bob Wald, Alan Wiseman, and the participants of the Third and Fourth Capra Meetings on Radiation Reaction for discussions that helped shape the presentation of this work.

\section{APPENDIX A: HADAMARD FORM OF THE SCALAR GREEN'S FUNCTION}

In this appendix we continue our discussion of the two-point function $A\left(x, x^{\prime}\right)$. While the results derived here are not required for the computation of the self-forces, they are interesting in their own right. Our main objective is to show that the scalar Green's function computed in Sec. III B can be cast in the Hadamard form 29]

$$
G\left(x, x^{\prime}\right)=\theta\left(x, x^{\prime}\right)\left[u\left(x, x^{\prime}\right) \delta(\sigma)+v\left(x, x^{\prime}\right) \theta(-\sigma)\right] .
$$

Here, $\sigma\left(x, x^{\prime}\right)$ is Synge's world function [61], equal to one-half the squared geodesic distance between $x$ and $x^{\prime} ; u\left(x, x^{\prime}\right)$ and $v\left(x, x^{\prime}\right)$ are two-point functions that are smooth at $\sigma=0$, and $\theta\left(x, x^{\prime}\right)$ is a time-ordering function, equal to 1 if $x$ is in the causal future of $x^{\prime}$, and zero otherwise. The calculations presented in this Appendix rely on methods introduced by Thorne and Kovacs [62]. 


\section{1. $A\left(x, x^{\prime}\right)$ on the light cone}

We go back to Eq. (4.6) and seek to evaluate $A\left(x, x^{\prime}\right)$ in the situation where the points $x$ and $x^{\prime}$ are linked by a null geodesic of the flat-spacetime background. In this situation $\Delta t=R$, or $s=e$. (Recall that $\Delta t=t-t^{\prime}$ and $R=\left|\boldsymbol{x}-\boldsymbol{x}^{\prime}\right|$.) This means that $\eta^{1}$ and $\eta^{2}$ are both zero, and that the vector of Eq. (4.5) reduces to $\boldsymbol{\eta}=\frac{1}{2} \cos \theta \boldsymbol{R}$. (Recall that the third axis is oriented along $\boldsymbol{R}=\boldsymbol{x}-\boldsymbol{x}^{\prime}$.) Integrating over $\phi$, we find that Eq. (4.6) becomes $A\left(x, x^{\prime}\right)=\frac{1}{2} \int_{-1}^{1} \Phi\left(\boldsymbol{x}_{0}+\boldsymbol{\eta}\right) d \cos \theta$. After changing the variable of integration to $\lambda=\frac{1}{2}(\cos \theta+1)$, this becomes

$$
A_{\mathrm{lc}}\left(x, x^{\prime}\right)=\int_{0}^{1} \Phi(\boldsymbol{\xi}(\lambda)) d \lambda
$$

where

$$
\xi(\lambda)=\boldsymbol{x}^{\prime}+\lambda\left(\boldsymbol{x}-\boldsymbol{x}^{\prime}\right)
$$

is a vector that interpolates linearly between $\boldsymbol{x}^{\prime}$ (when $\lambda=0$ ) and $\boldsymbol{x}$ (when $\lambda=1$ ). In Eq. (A2) we have indicated with the label "lc" that $A\left(x, x^{\prime}\right)$ is evaluated on the light cone. We see that $A_{\mathrm{lc}}\left(x, x^{\prime}\right)$ is the average of the Newtonian potential $\Phi$ over the straight line joining $\boldsymbol{x}$ and $\boldsymbol{x}^{\prime}$.

Up to now, our view of the two-point function $A_{\mathrm{lc}}\left(x, x^{\prime}\right)$ has been that it is the restriction of $A\left(x, x^{\prime}\right)$ on the light cone: the points $x$ and $x^{\prime}$ must be related by a null geodesic of the background Minkowski spacetime. The right-hand side of Eq. (A2), however, stays meaningful even for points that are not so related, and we take this opportunity to extend the definition of $A_{\mathrm{lc}}\left(x, x^{\prime}\right)$ to arbitrarily related points. This will be our new point of view: The two-point function $A_{\mathrm{lc}}\left(x, x^{\prime}\right)$ shall be defined for arbitrary points $x$ and $x^{\prime}$ by Eq. (A2). In the case where $x$ and $x^{\prime}$ are null related, then $A_{\mathrm{lc}}\left(x, x^{\prime}\right)=A\left(x, x^{\prime}\right)$.

We now show that $A_{\mathrm{lc}}\left(x, x^{\prime}\right)$, as defined for arbitrary points by Eq. A2), is intimately related to the world function $\sigma\left(x, x^{\prime}\right)$, defined by [62]

$$
\sigma\left(x, x^{\prime}\right)=\frac{1}{2} \int_{0}^{1} g_{\alpha \beta}\left(\xi^{\mu}\right) \frac{d \xi^{\alpha}}{d \lambda} \frac{d \xi^{\beta}}{d \lambda} d \lambda,
$$

where $\xi^{\alpha}(\lambda)$ describes the (unique) geodesic linking the points $x$ and $x^{\prime} ; \lambda$ is an affine parameter scaled in such a way that $\xi^{\alpha}(0)=x^{\alpha^{\prime}}$ and $\xi^{\alpha}(1)=x^{\alpha}$. The world function is to be calculated with the metric of Eq. (2.3).

In Eq. (A4), the geodesic $\gamma$ differs from a straight line by a quantity of order $\Phi$. Because Eq. (A4) is an action principle for the geodesic equation, this error of the first order in the specification of the curve produces an error of the second order in $\sigma$. To calculate $\sigma$ to first order in $\Phi$, it is sufficient to take $\gamma$ to be a straight line. The appropriate relations are then $\xi^{\alpha}(\lambda)=x^{\alpha^{\prime}}+\lambda\left(x^{\alpha}-x^{\alpha^{\prime}}\right)$. Substituting also $g_{\alpha \beta}=\eta_{\alpha \beta}-2 \Phi(\boldsymbol{\xi}) \chi_{\alpha \beta}$ in Eq. (A4), we obtain

$$
\sigma\left(x, x^{\prime}\right)=\sigma^{\text {flat }}\left(x, x^{\prime}\right)-\left(\Delta t^{2}+R^{2}\right) \int_{0}^{1} \Phi(\boldsymbol{\xi}(\lambda)) d \lambda,
$$

where $\sigma^{\text {flat }}\left(x, x^{\prime}\right)=\frac{1}{2}\left(-\Delta t^{2}+R^{2}\right)$.

Comparing Eqs. (A2) and A5, we arrive at

$$
\sigma\left(x, x^{\prime}\right)=\sigma^{\text {flat }}\left(x, x^{\prime}\right)-\left(\Delta t^{2}+R^{2}\right) A_{\mathrm{lc}}\left(x, x^{\prime}\right) .
$$

We see that the two-point function $A_{\mathrm{lc}}\left(x, x^{\prime}\right)$ determines by how much the squared geodesic interval $\sigma$ differs from its flat-spacetime value.

\section{2. van Vleck determinant}

The scalarized van Vleck determinant [41,63] plays a fundamental role in the theory of Green's functions in curved spacetime. This is defined by

$$
\Delta\left(x, x^{\prime}\right)=-\frac{\operatorname{det}\left[-\partial_{\alpha \beta^{\prime}} \sigma\right]}{\sqrt{g(x) g\left(x^{\prime}\right)}},
$$


where $\sigma\left(x, x^{\prime}\right)$ is the world function defined in Eq. (A4), and $g(x)$ is the metric determinant evaluated at $x$. The general theory predicts that in Eq. (A1), $u\left(x, x^{\prime}\right)$ is the square root of the van Vleck determinant. We will verify this result with our weak-curvature scalar Green's function. In order to do so we must compute $\Delta\left(x, x^{\prime}\right)$.

We rewrite Eq. (A6) in the form $\sigma=\sigma^{\text {flat }}-\Omega$, where

$$
\Omega\left(x, x^{\prime}\right)=\chi_{\mu \nu}\left(x-x^{\prime}\right)^{\mu}\left(x-x^{\prime}\right)^{\nu} A_{\mathrm{lc}}\left(x, x^{\prime}\right) .
$$

A straightforward computation, using $\sqrt{-g(x)}=1-2 \Phi(\boldsymbol{x})$ and $\partial_{\alpha \beta^{\prime}} \sigma^{\text {flat }}=-\eta_{\alpha \beta}$, reveals that to first order in the Newtonian potential, the van Vleck determinant is given by

$$
\Delta=1+2 \Phi(\boldsymbol{x})+2 \Phi\left(\boldsymbol{x}^{\prime}\right)+\eta^{\alpha \beta} \partial_{\alpha \beta^{\prime}} \Omega .
$$

The last term is evaluated from Eq. (A8), and we obtain

$$
\eta^{\alpha \beta} \partial_{\alpha \beta^{\prime}} \Omega=\left(\Delta t^{2}+R^{2}\right) \delta^{a b} \partial_{a b^{\prime}} A_{\mathrm{lc}}-2\left(x-x^{\prime}\right)^{a}\left(\partial_{a}-\partial_{a^{\prime}}\right) A_{\mathrm{lc}}-4 A_{\mathrm{lc}},
$$

where we have used the fact that $A_{\mathrm{lc}}\left(x, x^{\prime}\right)$, as defined by Eqs. (A2) and ( $\mathrm{A} 3$ ), depends only on the spatial variables $\boldsymbol{x}$ and $\boldsymbol{x}^{\prime}$. Calculating the derivatives is easy, and we get $\partial_{a} A_{\mathrm{lc}}=\int \lambda \Phi_{, a} d \lambda, \partial_{a^{\prime}} A_{\mathrm{lc}}=\int(1-\lambda) \Phi_{, a} d \lambda$, and $\partial_{a b^{\prime}} A_{\mathrm{lc}}=$ $\int \lambda(1-\lambda) \Phi_{, a b} d \lambda$. Inside the integrals, the derivatives of $\Phi$ are taken with respect to $\boldsymbol{\xi}$, and the various factors involving $\lambda$ come from differentiating this vector with respect to either $\boldsymbol{x}$ or $\boldsymbol{x}^{\prime}$. Substituting this into Eq. A10, we have

$$
\eta^{\alpha \beta} \partial_{\alpha \beta^{\prime}} \Omega=\left(\Delta t^{2}+R^{2}\right) \int_{0}^{1} \lambda(1-\lambda) \nabla^{2} \Phi d \lambda-2\left(x-x^{\prime}\right)^{a} \int_{0}^{1}(2 \lambda-1) \Phi,{ }_{a} d \lambda-4 \int_{0}^{1} \Phi d \lambda .
$$

In the first integral we replace $\nabla^{2} \Phi$ by $4 \pi \rho$. In the second integral we replace $\Phi_{, a}\left(x-x^{\prime}\right)^{a}$ by $d \Phi / d \lambda$, which allows us to integrate by parts. After simplification, we arrive at

$$
\eta^{\alpha \beta} \partial_{\alpha \beta^{\prime}} \Omega=4 \pi\left(\Delta t^{2}+R^{2}\right) \int_{0}^{1} \lambda(1-\lambda) \rho d \lambda-2 \Phi(\boldsymbol{x})-2 \Phi\left(\boldsymbol{x}^{\prime}\right) .
$$

Substituting this into Eq. (A9), we obtain our final expression for the van Vleck determinant:

$$
\Delta\left(x, x^{\prime}\right)=1+4 \pi\left(\Delta t^{2}+R^{2}\right) \int_{0}^{1} \lambda(1-\lambda) \rho(\boldsymbol{\xi}(\lambda)) d \lambda .
$$

We see that $\Delta\left(x, x^{\prime}\right)$ differs from 1 if and only if the straight line connecting the points $\boldsymbol{x}$ and $\boldsymbol{x}^{\prime}$ passes through the matter distribution.

\section{3. $A\left(x, x^{\prime}\right)$ near the light cone}

We have previously evaluated the two-point function $A\left(x, x^{\prime}\right)$ in the case where $x$ and $x^{\prime}$ are connected by a null geodesic of the background Minkowski spacetime. We now improve on this result, by allowing the parameter

$$
\varepsilon \equiv \sqrt{s^{2}-e^{2}}=\frac{1}{2} \sqrt{\Delta t^{2}-R^{2}}
$$

to be nonzero. We will, however, assume that $\varepsilon \ll 1$, and evaluate Eq. (4.6) in this limit. This is the near-light-cone approximation.

To second order in $\varepsilon$, the vector $\boldsymbol{\eta}$ of Eq. (4.5) reads $\eta^{1}=\varepsilon \sin \theta \cos \phi, \eta^{2}=\varepsilon \sin \theta \sin \phi$, and $\eta^{3}=e\left[1+\frac{1}{2}(\varepsilon / e)^{2}+\right.$ $\left.O\left(\varepsilon^{4}\right)\right] \cos \theta$. We substitute this into $\Phi\left(\boldsymbol{x}_{\mathbf{0}}+\boldsymbol{\eta}\right)$, expand to second order in $\varepsilon$, and then integrate over the angles. After re-introducing the parameter $\lambda=\frac{1}{2}(\cos \theta+1)$, we obtain

$$
A=A_{\mathrm{lc}}+4 \pi \varepsilon^{2} \int_{0}^{1} \lambda(1-\lambda) \rho d \lambda+\frac{\varepsilon^{2}}{2 e} \int_{0}^{1}(2 \lambda-1) \Phi_{, a} \hat{n}^{a} d \lambda-\varepsilon^{2} \int_{0}^{1} \lambda(1-\lambda) \Phi_{, a b} \hat{n}^{a} \hat{n}^{b} d \lambda+O\left(\varepsilon^{4}\right) .
$$

Here, $A_{\mathrm{lc}}$ is the restriction of $A\left(x, x^{\prime}\right)$ on the light cone, the quantity given by Eq. (A2), and $\hat{\boldsymbol{n}}$ is a unit vector pointing in the direction of $\boldsymbol{x}-\boldsymbol{x}^{\prime}$. The quantities inside the integrals are evaluated at the point $\boldsymbol{\xi}(\lambda)$ defined by Eq. (A3), and 
the derivatives of $\Phi$ are taken with respect to this vector. To get the first integral we have used Poisson's equation, $\nabla^{2} \Phi=4 \pi \rho$.

In the second and third integrals of Eq. (A15), the derivatives of $\Phi$ in the direction of $\hat{\boldsymbol{n}}$ can be expressed as derivatives with respect to $\lambda: d \Phi / d \lambda=2 e \Phi,{ }_{a} \hat{n}^{a}$ and $d^{2} \Phi / d \lambda^{2}=(2 e)^{2} \Phi, a b \hat{n}^{a} \hat{n}^{b}$. After substitution into Eq. (A15) and an integration by parts on the last term, we find that these integrals cancel out. Our final expression for the two-point function is therefore

$$
A\left(x, x^{\prime}\right)=A_{\mathrm{lc}}\left(x, x^{\prime}\right)+4 \pi \varepsilon^{2} \int_{0}^{1} \lambda(1-\lambda) \rho(\boldsymbol{\xi}(\lambda)) d \lambda+O\left(\varepsilon^{4}\right) .
$$

We recognize here the same integral over the mass density that appears in our previous expression for the van Vleck determinant, Eq. (A13). This allows us to rewrite Eq. (A16) as

$$
A\left(x, x^{\prime}\right)=A_{\mathrm{lc}}\left(x, x^{\prime}\right)+\frac{\Delta t^{2}-R^{2}}{8 R^{2}}\left[\Delta\left(x, x^{\prime}\right)-1\right]+O\left(\varepsilon^{4}\right) .
$$

In this calculation we have used the fact that the factor $\Delta t^{2}+R^{2}$ appearing on the right-hand side of Eq. (A13) is equal to $2 R^{2}\left[1+O\left(\varepsilon^{2}\right)\right]$.

Equations (A16) and A17) indicate that unless the straight line connecting $\boldsymbol{x}$ to $\boldsymbol{x}^{\prime}$ intersects the matter distribution, $A_{\mathrm{lc}}\left(x, x^{\prime}\right)$ makes an excellent approximation to $A\left(x, x^{\prime}\right)$.

\section{Scalar Green's function}

We now have the necessary tools to cast the scalar Green's function calculated in Sec. III B into the Hadamard form [29] displayed in Eq. (A1). Recall from Eq. (3.11) and (3.14) that our expression for the Green's function was

$$
G(x, x)=G^{\text {flat }}\left(x, x^{\prime}\right)-2 \partial_{t t^{\prime}} A\left(x, x^{\prime}\right)-2 \xi B\left(x, x^{\prime}\right)
$$

where $G^{\text {flat }}\left(x, x^{\prime}\right)$ is the retarded Green's function of flat spacetime, given by Eq. (3.3). This can be re-expressed as

$$
G^{\text {flat }}\left(x, x^{\prime}\right)=\theta\left(x, x^{\prime}\right) \delta\left(\sigma^{\text {flat }}\right)
$$

where $\theta\left(x, x^{\prime}\right)$ is the time ordering function introduced in Eq. (A1), and $\sigma^{\text {flat }}\left(x, x^{\prime}\right)=\frac{1}{2}\left(-\Delta t^{2}+R^{2}\right)$.

To calculate the Green's function we need to take into account the fact that both $A\left(x, x^{\prime}\right)$ and $B\left(x, x^{\prime}\right)$ are zero if $x$ and $x^{\prime}$ are spacelike related (in the flat-spacetime background), and if $x^{\prime}$ lies to the future of $x$. We express this as

$$
A\left(x, x^{\prime}\right)=\theta\left(x, x^{\prime}\right) \theta\left(-\sigma^{\text {flat }}\right) \hat{A}\left(x, x^{\prime}\right), \quad B\left(x, x^{\prime}\right)=\theta\left(x, x^{\prime}\right) \theta\left(-\sigma^{\text {flat }}\right) \hat{B}\left(x, x^{\prime}\right)
$$

in which the $\theta$-functions explicitly enforce the vanishing of the two-point functions except when $x^{\prime}$ is in the causal past of $x$. In what follows we will keep the time-ordering function implicit, and re-insert it at the end of the calculation.

Differentiation of Eq. (A20) gives

$$
\partial_{t t^{\prime}} A=\theta\left(-\sigma^{\mathrm{flat}}\right) \partial_{t t^{\prime}} \hat{A}-\delta\left(\sigma^{\mathrm{flat}}\right)\left(1+2 \Delta t \partial_{t}\right) \hat{A}+\delta^{\prime}\left(\sigma^{\mathrm{flat}}\right) \Delta t^{2} \hat{A}
$$

where the prime on the $\delta$-function indicates differentiation with respect to $\sigma^{\text {flat }}$. To calculate the time derivative of $\hat{A}$ in the second term, we use Eq. (A16) which we copy as $\hat{A}=A_{\mathrm{lc}}-\sigma^{\text {flat }} C+O\left(\sigma^{\text {flat } 2}\right)$, where $C=2 \pi \int \lambda(1-\lambda) \rho d \lambda$. Dropping all terms proportional to $\sigma^{\text {flat }}$, we find that the coefficient of the $\delta$-function becomes $-A_{\mathrm{lc}}-\chi C$, where $\chi \equiv \Delta t^{2}+R^{2}$. Working now on the third term, we find that the coefficient of the differentiated $\delta$-function is $\frac{1}{2} \chi A_{\mathrm{lc}}-\left(A_{\mathrm{lc}}+\frac{1}{2} \chi C\right) \sigma^{\text {flat }}+O\left(\sigma^{\text {flat } 2}\right)$. The term that is linear in $\sigma^{\text {flat }}$ can be transferred to the coefficient of the $\delta$-function with the help of the distributional identity $\sigma \delta^{\prime}(\sigma)=-\delta(\sigma)$. The end result is

$$
\partial_{t t^{\prime}} A=\theta\left(-\sigma^{\mathrm{flat}}\right) \partial_{t t^{\prime}} \hat{A}-\frac{1}{2} \chi C \delta\left(\sigma^{\mathrm{flat}}\right)+\frac{1}{2} \chi A_{\mathrm{lc}} \delta^{\prime}\left(\sigma^{\mathrm{flat}}\right) .
$$

We substitute this into Eq. (A18). With the help of Eqs. (A19) and (A20), we obtain

$$
G=(1+\chi C) \delta\left(\sigma^{\mathrm{flat}}\right)-\chi A_{\mathrm{lc}} \delta^{\prime}\left(\sigma^{\mathrm{flat}}\right)+\theta\left(-\sigma^{\mathrm{flat}}\right)\left[-2 \partial_{t t^{\prime}} \hat{A}-2 \xi \hat{B}\right] .
$$


We can re-express the first three terms in the form $(1+\chi C) \delta\left(\sigma^{\text {flat }}-\chi A_{\mathrm{lc}}\right)$. From Eq. (A6) we recognize the new $\operatorname{argument}$ of the $\delta$-function as the world function $\sigma\left(x, x^{\prime}\right)$ of the weakly curved spacetime. And we recognize the factor $1+\chi C$ in front as the square root of the van Vleck determinant, calculated in Eq. (A13). Finally, because the quantity within the square brackets in Eq. (A23) is already of the first order in the Newtonian potential, we can safely replace $\sigma^{\text {flat }}$ with $\sigma$ as the argument of the $\theta$-function. The only remaining task is to re-insert the time-ordering function that has been left out of our expressions.

Our conclusion is that the scalar Green's function of Eq. (A18) can indeed be cast in the form of Eq. (A1), with $u=\Delta^{1 / 2}$. For the weakly curved spacetimes considered in this paper, the world function $\sigma\left(x, x^{\prime}\right)$ is worked out in Eq. ( $(\overline{A 6})$, the van Vleck determinant $\Delta\left(x, x^{\prime}\right)$ in Eq. (A13), and

$$
v\left(x, x^{\prime}\right)=-2 \partial_{t t^{\prime}} \hat{A}\left(x, x^{\prime}\right)-2 \xi \hat{B}\left(x, x^{\prime}\right)
$$

is the tail part of the Green's function. Here, $\hat{A}\left(x, x^{\prime}\right)$ and $\hat{B}\left(x, x^{\prime}\right)$ are the two-point functions introduced in Sec. III A.

\section{APPENDIX B: TWO-POINT FUNCTIONS FOR THE POINT-MASS POTENTIAL}

In this Appendix we evaluate the two-point functions $A\left(x, x^{\prime}\right)$ and $B\left(x, x^{\prime}\right)$ for the special case $\Phi(\boldsymbol{x})=-M /|\boldsymbol{x}|$. The results derived here were first obtained by DeWitt and DeWitt [48]; we include this discussion here for completeness.

We begin with Eq. (4.6), which we rewrite as

$$
A\left(x, x^{\prime}\right)=-\frac{M}{4 \pi} \int \frac{1}{\left|\boldsymbol{\eta}-\boldsymbol{\eta}_{0}\right|} d \Omega,
$$

where $\boldsymbol{\eta}(s, \theta, \phi)$ is the vector of Eq. (4.5) with $s=\frac{1}{2} \Delta t \equiv \frac{1}{2}\left(t-t^{\prime}\right)$, and $\boldsymbol{\eta}_{0} \equiv-\boldsymbol{x}_{0} \equiv-\frac{1}{2}\left(\boldsymbol{x}+\boldsymbol{x}^{\prime}\right)$. The point $\boldsymbol{\eta}_{0}$, at which the central mass is located (in a coordinate system centered at $\boldsymbol{\eta}=0$ ), will be represented by the ellipsoidal coordinates $\left(s_{0}, \theta_{0}, \phi_{0}\right)$. We recall that $e=\frac{1}{2} R=\frac{1}{2}\left|\boldsymbol{x}-\boldsymbol{x}^{\prime}\right|$ is the ellipticity of the coordinate system.

To evaluate the integral of Eq. (B1) we invoke the addition theorem in ellipsoidal coordinates [64],

$$
\frac{1}{\left|\boldsymbol{\eta}-\boldsymbol{\eta}_{0}\right|}=\frac{4 \pi}{e} \sum_{l=0}^{\infty} \sum_{m=-l}^{l}(-1)^{m} \frac{(l-m) !}{(l+m) !} P_{l}^{m}\left(s_{<} / e\right) Q_{l}^{m}\left(s_{>} / e\right) Y_{l m}^{*}\left(\theta_{0}, \phi_{0}\right) Y_{l m}(\theta, \phi),
$$

where $s_{<}\left(s_{>}\right)$is the lesser (greater) of $s$ and $s_{0}$, and $P_{l}^{m}$ and $Q_{l}^{m}$ are associated Legendre polynomials [65]. Substituting this into Eq. (B1), we find that the integration over the spherical harmonics $Y_{l m}(\theta, \phi)$ is zero unless $l$ and $m$ are both zero, and we obtain $A=-(M / e) P_{0}\left(s_{<} / e\right) Q_{0}\left(s_{>} / e\right)$, or

$$
A=-\frac{M}{R} \ln \frac{2 s_{>}+R}{2 s_{>}-R}
$$

using the known forms for the Legendre functions of zeroth order.

The hard part of the calculation resides with the computation of $s_{0}$. Recalling that $\boldsymbol{x}$ and $\boldsymbol{x}^{\prime}$ differ by a vector $\boldsymbol{R}$ pointing in the $z$ direction, we write $\boldsymbol{x}=(x, y, z), \boldsymbol{x}^{\prime}=(x, y, z-R)$, and we have $\boldsymbol{\eta}_{0}=\left(-x,-y,-z+\frac{1}{2} R\right)$. This and Eq. (4.5) give us the equations

$$
\sqrt{s_{0}^{2}-e^{2}} \sin \theta_{0} \cos \phi_{0}=-x, \quad \sqrt{s_{0}^{2}-e^{2}} \sin \theta_{0} \sin \phi_{0}=-y, \quad s_{0} \cos \theta_{0}=-z+e,
$$

which must be solved for $s_{0}$. It is not hard to show that this amounts to solving the quadratic

$$
s_{0}^{4}-\frac{1}{2}\left(r^{2}+r^{\prime 2}\right) s_{0}^{2}+\frac{1}{16}\left(r^{2}-r^{\prime 2}\right)^{2}=0,
$$

where $r=|\boldsymbol{x}|$ and $r^{\prime}=\left|\boldsymbol{x}^{\prime}\right|$. This finally gives $s_{0}=\frac{1}{2}\left(r+r^{\prime}\right)$.

Going back to Eq. (B3), we see that we must distinguish between the cases $\Delta t>r+r^{\prime}$, for which $2 s_{>}=\Delta t$, and $\Delta t<r+r^{\prime}$, for which $2 s_{>}=r+r^{\prime}$. This gives

$$
A\left(x, x^{\prime}\right)=-\frac{M}{R} \theta(\Delta t-R)\left[\theta\left(r+r^{\prime}-\Delta t\right) \ln \frac{r+r^{\prime}+R}{r+r^{\prime}-R}+\theta\left(\Delta t-r-r^{\prime}\right) \ln \frac{\Delta t+R}{\Delta t-R}\right],
$$


where, we recall, $r=|\boldsymbol{x}|, r^{\prime}=\left|\boldsymbol{x}^{\prime}\right|, R=\left|\boldsymbol{x}-\boldsymbol{x}^{\prime}\right|$, and $\Delta t=t-t^{\prime}$. For completeness we have re-inserted the step function $\theta(\Delta t-R)$ that was left implicit in Eqs. (4.6) and (B1). We see that the two-point function undergoes a change of behavior when $\Delta t=r+r^{\prime}$. This time delay corresponds to a signal propagating with the speed of light from $\boldsymbol{x}$ to the center (at which the Newtonian potential is singular) and then on to $\boldsymbol{x}^{\prime}$. For shorter delays, $A\left(x, x^{\prime}\right)$ is time-independent; for longer delays, $A\left(x, x^{\prime}\right)$ depends explicitly on $\Delta t$. This sudden change of behavior makes the two-point function slightly suspicious: Although our calculations are based on the assumption that the spacetime is weakly curved everywhere, the change of behavior is dictated by a region of spacetime - the center - in which the Newtonian potential is decidedly not small.

The computation of $B\left(x, x^{\prime}\right)$ is quite simple for the potential $\Phi=-M / r$, for which the mass density is $\rho(\boldsymbol{x})=$ $M \delta(\boldsymbol{x})$. Substituting this into Eq. (4.2) gives

$$
B\left(x, x^{\prime}\right)=\frac{M}{r r^{\prime}} \delta\left(\Delta t-r-r^{\prime}\right) .
$$

Again we notice a suspicious dependence on the conditions at the center.

Despite the notes of caution, the results derived here for the special case $\Phi=-M / r$ are in complete agreement with our general results of Sec. IV. First, Eq. (B7) is identical to Eq. (4.18), which was obtained as the leading term in a multipole expansion for $B\left(x, x^{\prime}\right)$. This indicates that in fact, our result for this two-point function is quite insensitive to the conditions near the center. Second, Eq. (B6) implies that near coincidence $(R$ small and smaller than $\Delta t)$, $A\left(x, x^{\prime}\right)$ can be approximated by

$$
A\left(x, x^{\prime}\right)=-\frac{2 M}{r+r^{\prime}}\left[1+\frac{1}{3}\left(\frac{R}{r+r^{\prime}}\right)^{2}+O\left(R^{4}\right)\right],
$$

which could be recast in the form of Eq. (4.11). In particular, differentiation of Eq. (B8) with respect to $\boldsymbol{x}$ and $\boldsymbol{x}^{\prime}$ confirms Eq. (4.13). In this case we have $\Phi_{, a b}=\left(M / r^{3}\right)\left(\delta_{a b}-3 r_{, a} r_{, b}\right)$, with $r_{, a}=x^{a} / r$. Third, Eq. (B6) implies that for long delays $(\Delta t$ large and larger than $R$ ), we have the approximation

$$
A\left(x, x^{\prime}\right)=-\frac{2 M}{\Delta t}\left[1+\frac{1}{3}\left(\frac{R}{\Delta t}\right)^{2}+O\left(\Delta t^{-4}\right)\right],
$$

which is evidently compatible with Eq. (4.14). This comparison between our general results of Sec. IV and those of this Appendix shows that any aspect of the two-point functions that might be sensitive to the strong-field portion of the spacetime near the center will not be involved in the computation of the self-forces. This statement provides a further validation of the work of DeWitt and DeWitt [48], which was entirely based on the special case $\Phi=-M / r$.

[1] A. Einstein, L. Infeld, and B. Hoffmann, Ann. Math. 39, 65 (1938).

[2] S. Chandrasekhar and F.P. Esposito, Astrophys. J. 160, 153 (1970).

[3] T. Damour, C. R. Acad. Sci. Paris 294, série II, 1355 (1982).

[4] G. Schäfer, Ann. Phys. (NY) 161, 81 (1985).

[5] L.P. Grishchuk and S.M. Kopejkin, in Relativity in Celestial Mechanics and Astrometry (IAU Symposium 114, Leningrad, 1985), edited by J. Kovalevsky and V.A. Brumberg (Reidel, Dordrecht, 1986).

[6] P. Jaranowski and G. Shäfer, Ann. Phys. (Leipzig) 9, 378 (2000).

[7] T. Damour, P. Jaranowski and G. Shäfer, Phys. Rev. D 63, 044021 (2001); gr-qc/0105038 (2001).

[8] L. Blanchet and G. Faye, Phys. Rev. D 63, 062005 (2001).

[9] V. de Andrade, L. Blanchet, and G. Faye, Class. Quantum. Grav. 18, 753 (2001).

[10] M.E. Pati and C.M. Will, Phys. Rev. D 62, 124015 (2000).

[11] T. Damour, in 300 Years of Gravitation, edited by S.W. Hawking and W. Israel (Cambridge University Press, Cambridge, 1987).

[12] P. Havas, in Einstein and the History of General Relativity, edited by D. Howard and J. Stachel (Birkhäuser, Boston, 1989).

[13] Consider flat-spacetime electrodynamics as an example. Here, the retarded vector potential $A_{\text {ret }}^{\alpha}$ can be decomposed as $\frac{1}{2}\left(A_{\mathrm{ret}}^{\alpha}+A_{\mathrm{adv}}^{\alpha}\right)+\frac{1}{2}\left(A_{\mathrm{ret}}^{\alpha}-A_{\mathrm{adv}}^{\alpha}\right)$, where $A_{\mathrm{adv}}^{\alpha}$ is the advanced potential. It can be shown that the first part is singular at the position of the particle, but that it does not influence its motion. The second part, on the other hand, is well behaved at the 
particle's location, and it gives rise to the Abrahams-Lorentz-Dirac radiation-reaction force. The analogous decomposition of the vector potential in curved spacetime is much more delicate.

[14] T. Tanaka, M. Shibata, M. Sasaki, H. Tagoshi, and T. Nakamura, Prog. Theor. Phys. 90, 65 (1993).

[15] C. Cutler, D. Kennefick, and E. Poisson, Phys. Rev. D 50, 3816 (1994).

[16] M. Shibata, Prog. Theor. Phys. 99, 595 (1993).

[17] F.D. Ryan, Phys. Rev. D 53, 3064 (1996).

[18] D. Kennefick and A. Ori, Phys. Rev. D 53, 4319 (1996).

[19] D. Kennefick, Phys. Rev. D 58, 064012 (1998).

[20] S.A. Hughes, Phys. Rev. D 61, 084004 (2000).

[21] A. Ori and K.S. Thorne, Phys. Rev. D 62, 124022 (2000).

[22] B. Carter, Phys. Rev. 174, 1559 (1968).

[23] L.S. Finn and K.S. Thorne, Phys. Rev. D 62, 124021 (2000).

[24] The LISA mission is described at http://lisa.jpl.nasa.gov/. A collection of relevant articles can be found in Laser Interferometer Space Antenna, Proceedings of the Second International LISA Symposium, AIP Conference Proceedings 456 (American Institute of Physics, Woodbury, 1998).

[25] The report is titled Astronomy and Astrophysics in the New Millennium. It can be found at http://books.nap.edu/books/0309070317/html/

[26] Y. Mino, M. Sasaki, and T. Tanaka, Phys. Rev. D 55, 3457 (1997).

[27] T.C. Quinn and R.M. Wald, Phys. Rev. D 56, 3381 (1997).

[28] D.W. Sciama, P.C. Waylen, and R.C. Gilman, Phys. Rev. 187, 1762 (1969).

[29] J. Hadamard, in Lectures on Cauchy's Problem in Linear Partial Differential Equations (Yale University Press, New Haven, 1923), shows that the Green's function can be decomposed into a "direct part" that has support on the past light cone of the field point $x$, and a "tail part" that has support inside the light cone. The direct part of the Green's function is singular, but the tail part is well behaved. This decomposition is meaningful only in a normal convex neighborhood of $x$, which means that $x$ and $x^{\prime}$ must be linked by a unique geodesic.

[30] P.L. Chrzanowski, Phys. Rev. D 11, 2042 (1975).

[31] A. Ori, Phys. Lett. A202, 347 (1995).

[32] L.M. Burko, Phys. Rev. Lett. 84, 4529 (2000); Class. Quantum Grav. 17, 227 (2000); Am. J. Phys. 68, 456 (2000).

[33] L. Barack and A. Ori, Phys. Rev. D 61, 061502 (2000); gr-qc/0107056 (2001).

[34] L. Barack, Phys. Rev. D 62, 084027 (2000); gr-qc/0105040 (2001).

[35] L. Barack and L.M. Burko, Phys. Rev. D 62, 084040 (2000).

[36] L.M. Burko, Y.T. Liu, and Y. Soen, Phys. Rev. D 63, 024015 (2001).

[37] L.M. Burko and Y.T. Liu, Phys. Rev. D 64, 024006 (2001).

[38] C.O. Lousto, Phys. Rev. Lett. 84, 5251 (2000).

[39] H. Nakano and M. Sasaki, Prog. Theor. Phys. 105, 197 (2001).

[40] H. Nakano, Y. Mino, and M. Sasaki, gr-qc/0104012 (2001).

[41] B.S. DeWitt and R.W. Brehme, Ann. Phys. (NY) 9, 220 (1960).

[42] J.M. Hobbs, Ann. Phys. (NY) 47, 141 (1968).

[43] See, for example, J.D. Jackson, Classical Electrodynamics (Wiley, New York, 1975).

[44] See E. Poisson, gr-qc/9912045 (1999) for a pedagogical introduction to the Abrahams-Lorentz-Dirac equation.

[45] The Abrahams-Lorentz-Dirac equation is usually expressed in terms of $\dot{a}^{\alpha}$, the proper-time derivative of the particle's acceleration. Within the approximation implied by the point-particle description, it is consistent to replace this by $\dot{f}_{\text {ext }}^{\alpha} / m$, which we do throughout the paper. This reduction-of-order of the equations of motion is reviewed in Ref. [44]. The procedure is explained and motivated in E.E. Flanagan and R.M. Wald, Phys. Rev. D 54, 6233 (1996).

[46] T.C. Quinn, Phys. Rev. D 62, 064029 (2000).

[47] A.G. Wiseman, Phys. Rev. D 61, 084014 (2000).

[48] B.S. DeWitt and C.M. DeWitt, Physics (Long Island City, NY) 1, 3 (1964).

[49] A.G. Smith and C.M. Will, Phys. Rev. D 22, 1276 (1980).

[50] The authors have been very slow at recognizing this simple point, and we gratefully acknowledge relevant conversations with Alan Wiseman and Bob Wald. Our confusion had to do with the fact that it ought to be possible to adopt a coordinate system in which the star always appears to be at rest (except for internal motions which we ignore). While undoubtedly true, this is (probably) incompatible with the gauge conditions imposed in our calculations. Indeed, the calculations presented in Sec. VI give rise to a description in which the star and the particle both move around a fixed center of mass.

[51] P. Havas and J.N. Goldberg, Phys. Rev. 128, 398 (1962).

[52] A correction of $n \mathrm{PN}$ order is a term of order $\Phi^{n}$ relative to the leading term, which is labelled "Newtonian". In the post-Newtonian expansion, it is normally assumed that $v^{2}=O(\Phi)$, where $v$ is the velocity of an object moving in the gravitational potential $\Phi$. A quantity such as $m^{2} d \boldsymbol{g} / d t$ is $1.5 \mathrm{PN}$ order relative to $m \boldsymbol{g}$ : if $r_{c}$ is a characteristic length scale and $t_{c}$ a characteristic time scale associated with the motion, then $m^{2} d \boldsymbol{g} / d t \sim\left(m / r_{c}\right)\left(r_{c} / t_{c}\right)(m \boldsymbol{g})=O(\Phi v) m \boldsymbol{g}$.

[53] M. Carmeli, Ann. Phys. (NY) 34, 465 (1965); 35, 250 (1965).

[54] See, for example, C.W. Misner, K.S. Thorne, and J.A. Wheeler, Gravitation (Freeman, San Francisco, 1973), Exercise 
39.15.

[55] A.G. Wiseman (personal communication). Wiseman's unpublished calculations were presented at the Second Capra Meeting on Radiation Reaction, in Dublin, Ireland (July 1999). The proceedings of this conference, which contain Wiseman's presentation, can be found at http://www.lsc-group.phys.uwm.edu/ patrick/ireland99/.

[56] R.M. Wald (personal communication).

[57] A.I. Zel'nikov and V.P. Frolov, Zh. Eksp. Teor. Fiz. 82, 321 (1982) [Sov. Phys. JETP 55(2), 191 (1982)].

[58] V.P. Frolov (personal communication).

[59] C. Morette-DeWitt and J.L. Ging, C. R. Acad. Sci. Paris 251, 1868 (1960).

[60] L.D. Landau and E.M. Lifshitz, The Classical Theory of Fields (Pergamond, Oxford, 1962), Section 105.

[61] J.L. Synge Relativity: The General Theory (North-Holland, Amsterdam, 1960).

[62] K.S. Thorne and S.J. Kovacs, Astrophys. J. 200, 245 (1975).

[63] J.H. van Vleck, Proc. Nat. Acad. Sci. (US) 14, 178 (1928).

[64] T.M. MacRobert, Spherical Harmonics (Methuen, London, 1927), Chap. XI.

[65] G. Arfken, Mathematical Methods for Physicists (Academic Press, Orlando, 1985), Chap. 12. 